Supporting Information for

\title{
A Highly Selective Manganese-Catalyzed Synthesis of Imines under Phosphine-Free Conditions
}

\author{
Huining Chai, *, ${ }^{\dagger}$, "Kun Yu, " "Bo Liu,, " " Weiqiang Tan, ${ }^{\dagger}$ and Guangyao Zhang ${ }^{\S}$ \\ †School of Environmental and Municipal Engineering, Qingdao University of Technology, \\ Qingdao 266033, People's Republic of China \\ \$School of Medicine and Life Sciences, University of Jinan-Shandong Academy of Medical \\ Sciences; Institute of Materia Medica, Shandong First Medical University \& Shandong Academy \\ of Medical Sciences, Jinan 250062, People's Republic of China \\ ${ }^{\S}$ Research Center for Intelligent and Wearable Technology, College of Textiles and Clothing, \\ Qingdao University, Qingdao 266071, People's Republic of China
}

E-mail: chaihuining@qut.edu.cn.

\section{$\underline{\text { Experimental procedures and analytical data }}$}

Contents:

page

1. General considerations

2. Experimental procedures

3. X-Ray crystallographic studies

4. Copies of NMR spectra 


\section{General considerations}

The solvents were dried and distilled prior to use by the literature methods. ${ }^{1} \mathrm{H}$ and ${ }^{13} \mathrm{C}\left\{{ }^{1} \mathrm{H}\right\}$ NMR spectra were recorded on a $600 \mathrm{MHz}$ spectrometer and all chemical shift values refer to $\mathrm{CDCl}_{3}\left(\delta\left({ }^{1} \mathrm{H}\right), 7.26 \mathrm{ppm} ; \delta\left({ }^{13} \mathrm{C}\right), 77.16 \mathrm{ppm}\right)$, DMSO- $d_{6}\left(\delta\left({ }^{1} \mathrm{H}\right), 2.50\right.$ ppm; $\left.\delta\left({ }^{13} \mathrm{C}\right), 39.52 \mathrm{ppm}\right), \mathrm{CD}_{3} \mathrm{COCD}_{3}\left(\delta\left({ }^{1} \mathrm{H}\right), 2.05 \mathrm{ppm} ; \delta\left({ }^{13} \mathrm{C}\right), 206.26\right.$ and $\left.29.84 \mathrm{ppm}\right)$ and $\mathrm{CD}_{3} \mathrm{OD}\left(\delta\left({ }^{1} \mathrm{H}\right), 3.31 \mathrm{ppm} ; \delta\left({ }^{13} \mathrm{C}\right), 49.00 \mathrm{ppm}\right)$. X-ray Crystallographic analysis was achieved by Shiyanjia Lab (www.shiyanjia.com). Analytical TLC plates were viewed by UV light $(254 \mathrm{~nm})$. Column chromatographic purifications were performed on silica gel 160. All the chemical reagents were purchased from commercial sources and used as received unless otherwise indicated. Compound $\mathbf{1 a}$ and $\mathbf{1 b}$ were prepared by the reported methods. ${ }^{1}$ Compounds $5 \mathbf{a}, \mathbf{5 b}, \mathbf{5 c}, \mathbf{5 g}, \mathbf{5 n}, \mathbf{5 p}, \mathbf{5 t}, \mathbf{5 a d}, \mathbf{5 a f}$ and $\mathbf{5 a j}{ }^{2}$ 5an, ${ }^{3} 5 u, 5 v$, and $5 w,{ }^{4} \mathbf{x}$, and 5aa, ${ }^{5}$ dd, 5l, 5m, 5o, and 5ae, ${ }^{6} 5$ r, and 5ac, ${ }^{7}$ e,${ }^{8} 5$ ag, and 5 al,${ }^{9} 5$ ak $,{ }^{10} \mathbf{5 k},{ }^{11} \mathbf{5 q},{ }^{12} \mathbf{5 z},{ }^{13} \mathbf{5 j},{ }^{14} \mathbf{5 h},{ }^{15} \mathbf{5 i},{ }^{16} \mathbf{5 a m},{ }^{17} \mathbf{5 a h},{ }^{18}$ 5ai, ${ }^{19}$ 5ab, 5an, and $\mathbf{5 a o},{ }^{20} \mathbf{5 f}$, and $\mathbf{5 y},{ }^{21}$ and $\mathbf{5} \mathbf{s}^{22}$ were known and their analytical data are consistent with those reported in the literature. 


\section{Experimental procedures}

\subsection{Typical procedures for the preparation of imines}

Screening of the reaction conditions: By means of the reaction of benzyl alcohol (3a) with aniline (4a) as the model reaction, the reaction conditions were optimized as follows.

Table S1. Screening of amount of $\mathbf{3 a} .^{a}$

\begin{tabular}{|c|c|c|c|}
\hline $3 a$ & $4 a$ & & \\
\hline Entry & Amount of $\mathbf{3 a}(\mathrm{mmo}$ & $\begin{array}{c}\text { Conv. }{ }^{b} \\
(\%)\end{array}$ & $\begin{array}{c}\text { 5a:5a' } \\
(\text { molar ratio })^{b} \\
(\%)\end{array}$ \\
\hline 1 & 0.2 & 92.3 & $96: 4$ \\
\hline 2 & 0.22 & 97 & 98.1:1.9 \\
\hline 3 & 0.24 & 97.5 & $87.7: 12.3$ \\
\hline
\end{tabular}

${ }^{a}$ Conditions: 4a $(0.20 \mathrm{mmol})$, complex catalyst 2a $(0.006 \mathrm{mmol})$, toluene $(2 \mathrm{~mL}), 110^{\circ} \mathrm{C}$, $0.1 \mathrm{MPa} \mathrm{N}_{2}, 24$ h. ${ }^{b}$ Determined by GC analysis.

Table S2. Screening of base loading. ${ }^{\text {a }}$

\begin{tabular}{|c|c|c|c|}
\hline $3 a$ & $4 a$ & & \\
\hline Entry & $\begin{array}{c}\text { Base loading } \\
\text { (equiv.) }\end{array}$ & $\begin{array}{c}\text { Conv. }^{b} \\
(\%)\end{array}$ & $\begin{array}{c}\mathbf{5 a}: \mathbf{5 a}{ }^{\prime} \\
(\text { molar ratio })^{b} \\
(\%)\end{array}$ \\
\hline 1 & 0.3 & 82 & $96: 4$ \\
\hline 2 & 0.4 & 90 & $96: 4$ \\
\hline 3 & 0.5 & 97 & 98.1:1.9 \\
\hline 4 & 0.6 & 97 & $97: 3$ \\
\hline
\end{tabular}

${ }^{a}$ Conditions: 3a $(0.22 \mathrm{mmol}), \mathbf{4 a}(0.20 \mathrm{mmol})$, complex catalyst $\mathbf{2 a}(0.006 \mathrm{mmol})$, toluene $(2 \mathrm{~mL}), 110{ }^{\circ} \mathrm{C}, 0.1 \mathrm{MPa} \mathrm{N}_{2}, 24$ h. ${ }^{b}$ Determined by GC analysis. 
Table S3. Screening of 4 A MS loading. ${ }^{a}$

\begin{tabular}{|c|c|c|c|c|}
\hline $3 \mathbf{a}$ & $4 a$ & & & Sa' \\
\hline Entry & $\begin{array}{c}4 \AA \text { MS } \\
\text { loading } \\
(\mathrm{mg})\end{array}$ & $\begin{array}{c}\text { Conv. }^{b} \\
(\%)\end{array}$ & $\begin{array}{c}\mathbf{5 a}: \mathbf{5 a} \\
\left(_{\text {molar ratio })^{b}}\right. \\
(\%)\end{array}$ & \\
\hline 1 & 200 & 2.5 & 100 & \\
\hline 2 & 100 & 16 & 100 & \\
\hline 3 & 50 & 5.58 & 100 & \\
\hline 4 & 20 & 28 & 100 & \\
\hline 5 & 10 & 85 & 100 & \\
\hline 6 & 5 & 97 & $99.5: 0.5$ & \\
\hline
\end{tabular}

${ }^{a}$ Conditions: 3a $(0.22 \mathrm{mmol}), \mathbf{4 a}(0.20 \mathrm{mmol})$, complex catalyst $\mathbf{2 a}(0.006 \mathrm{mmol})$, toluene $(2 \mathrm{~mL}), 110{ }^{\circ} \mathrm{C}, 0.1 \mathrm{MPa} \mathrm{N}_{2}, 24$ h. ${ }^{b}$ Determined by GC analysis.

\subsection{Mechanism studies}

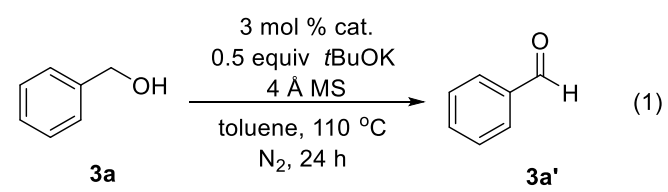

Dehydrogenation of phenylmethanol (3a): Under a nitrogen atmosphere a mixture of phenylmethanol (3a) $(56.9 \mu \mathrm{L}, 0.55 \mathrm{mmol})$, complex $2 \mathbf{a}(5.5 \mathrm{mg}, 0.015$ mmol), $4 \AA \mathrm{MS}(12.5 \mathrm{mg})$ and $t \mathrm{BuOK}(28.1 \mathrm{mg}, 0.25 \mathrm{mmol})$ in $2.0 \mathrm{~mL}$ toluene was stirred at $110{ }^{\circ} \mathrm{C}$ for $24 \mathrm{~h}$, leading to the quantitative formation of benzaldehyde (3a') which was analyzed by GC analysis (Eq. (1)).

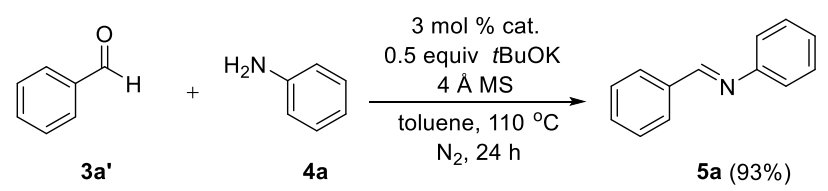

Condensation of the aldehyde intermediate with aniline: Under a nitrogen atmosphere a mixture of benzaldehyde (3a') (0.55 mmol), aniline (4a) (45.6 $\mu \mathrm{L}, 0.2$ mmol), complex 2a (5.5 mg, $0.015 \mathrm{mmol}), 4$ Å MS (12.5 mg) and $t \mathrm{BuOK}$ (28.1 mg, $0.25 \mathrm{mmol}$ ) in $2.0 \mathrm{~mL}$ toluene was stirred at $110{ }^{\circ} \mathrm{C}$ for $24 \mathrm{~h}$. After cooling to ambient 
temperature, the reaction was quenched with $10 \mathrm{~mL}$ water and extracted with EtOAc $(3 \times 10 \mathrm{~mL})$. The combined organic phase was concentrated under reduced pressure. The resultant residue was subject to purification by column chromatography on silica gel (eluent: $\mathrm{n}$-hexane/ethyl ether $=20: 1, \mathrm{v} / \mathrm{v}$ ) to afford $\mathbf{5 a}$ as a white solid $(84.3 \mathrm{mg}, 93 \%$ ). (Eq. (2)).

\section{X-Ray crystallographic studies}

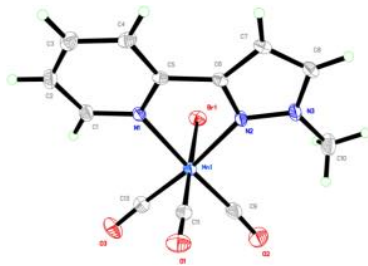

Figure S1. Molecular structure of complex 2b.

Table S4. Crystal data and structure refinement for complex $\mathbf{2 b}$.

Identification code

Empirical formula

Formula weight

Temperature

Wavelength

Crystal system

Space group

Unit cell dimensions

Volume

$\mathrm{Z}$

Density (calculated)

Absorption coefficient

$\mathrm{F}(000)$

Crystal size

Theta range for data collection

Index ranges

Reflections collected

Independent reflections

Completeness to theta $=25.009^{\circ}$

Refinement method mo_20190527znz2482_0m_a

C13 H9 Br Mn N3 O3

376.07

193(2) K

$0.71073 \AA$

Monoclinic

$\mathrm{P} 21 / \mathrm{n}$

$\mathrm{a}=7.2386(16) \AA \quad \alpha=90^{\circ}$.

$\mathrm{b}=9.670(2) \AA$

$\beta=84.184(4)^{\circ}$.

$\mathrm{c}=19.537(4) \AA$

$\gamma=90^{\circ}$

1360.4(5) $\AA^{3}$

4

$1.836 \mathrm{Mg} / \mathrm{m}^{3}$

$3.914 \mathrm{~mm}^{-1}$

740

$0.180 \times 0.160 \times 0.110 \mathrm{~mm}^{3}$

2.096 to $25.009^{\circ}$.

$-8<=\mathrm{h}<=7,-11<=\mathrm{k}<=11,-23<=\mathrm{l}<=23$

9945

$2392[\mathrm{R}(\mathrm{int})=0.0449]$

$99.5 \%$

Full-matrix least-squares on $\mathrm{F}^{2}$ 
Data / restraints / parameters

Goodness-of-fit on $\mathrm{F}^{2}$

Final $\mathrm{R}$ indices [I $>2 \operatorname{sigma}(\mathrm{I})]$

$\mathrm{R}$ indices (all data)

Extinction coefficient

Largest diff. peak and hole
2392 / 0 / 182

1.064

$\mathrm{R} 1=0.0252, \mathrm{wR} 2=0.0562$

$\mathrm{R} 1=0.0320, \mathrm{wR} 2=0.0584$

$\mathrm{n} / \mathrm{a}$

0.410 and -0.420 e. $\AA^{-3}$

\section{Copies of NMR spectra}
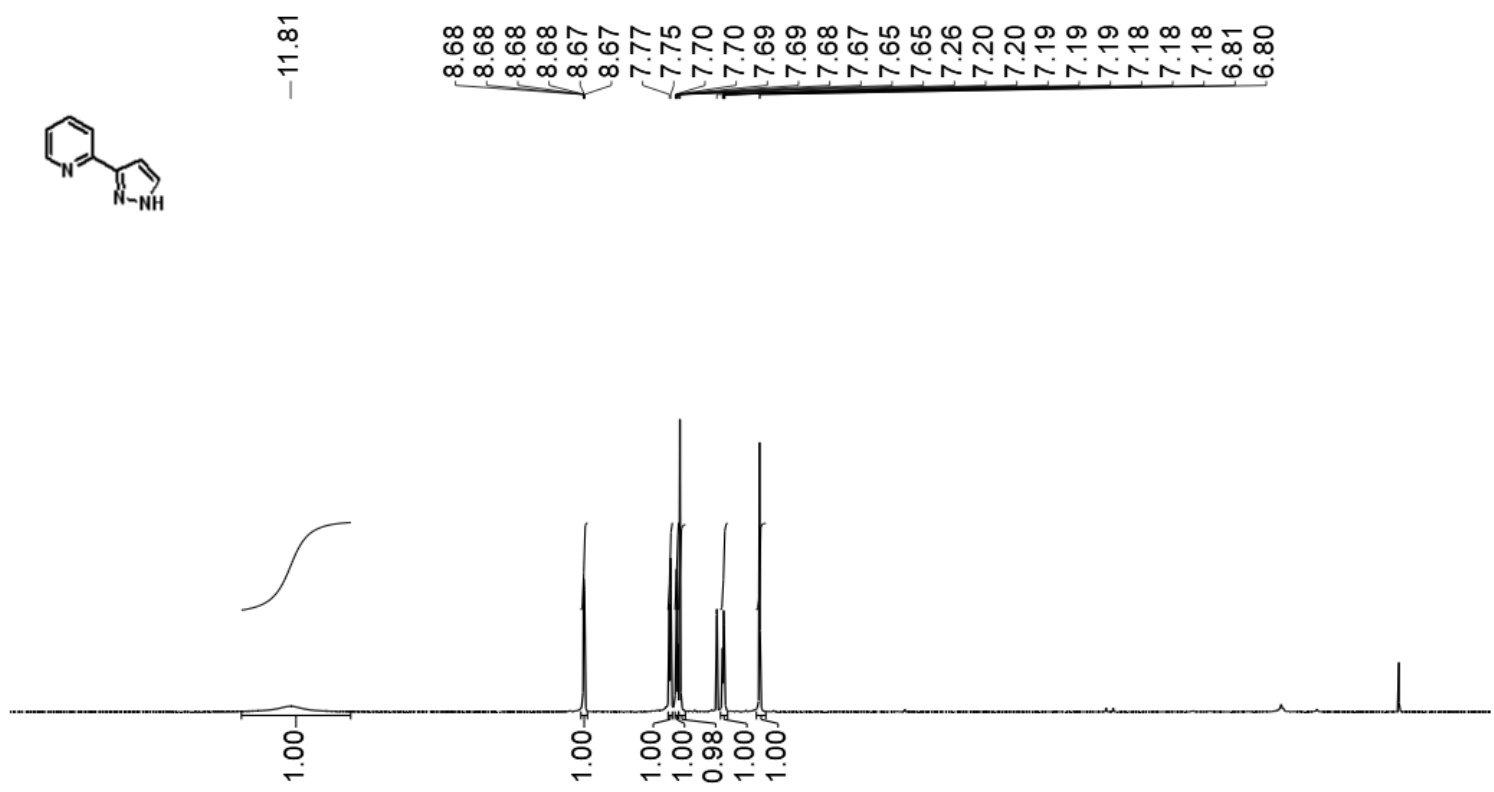

$\begin{array}{lllllllllllllll}14 & 13 & 12 & 11 & 10 & 9 & 8 & 7 & 6 & 5 & 4 & 3 & 2 & 1 & 0\end{array}$

Figure S2. ${ }^{1} \mathrm{H}$ NMR spectrum of $1 \mathbf{a}\left(\mathrm{CDCl}_{3}, 600 \mathrm{MHz}, 23{ }^{\circ} \mathrm{C}\right)$. 

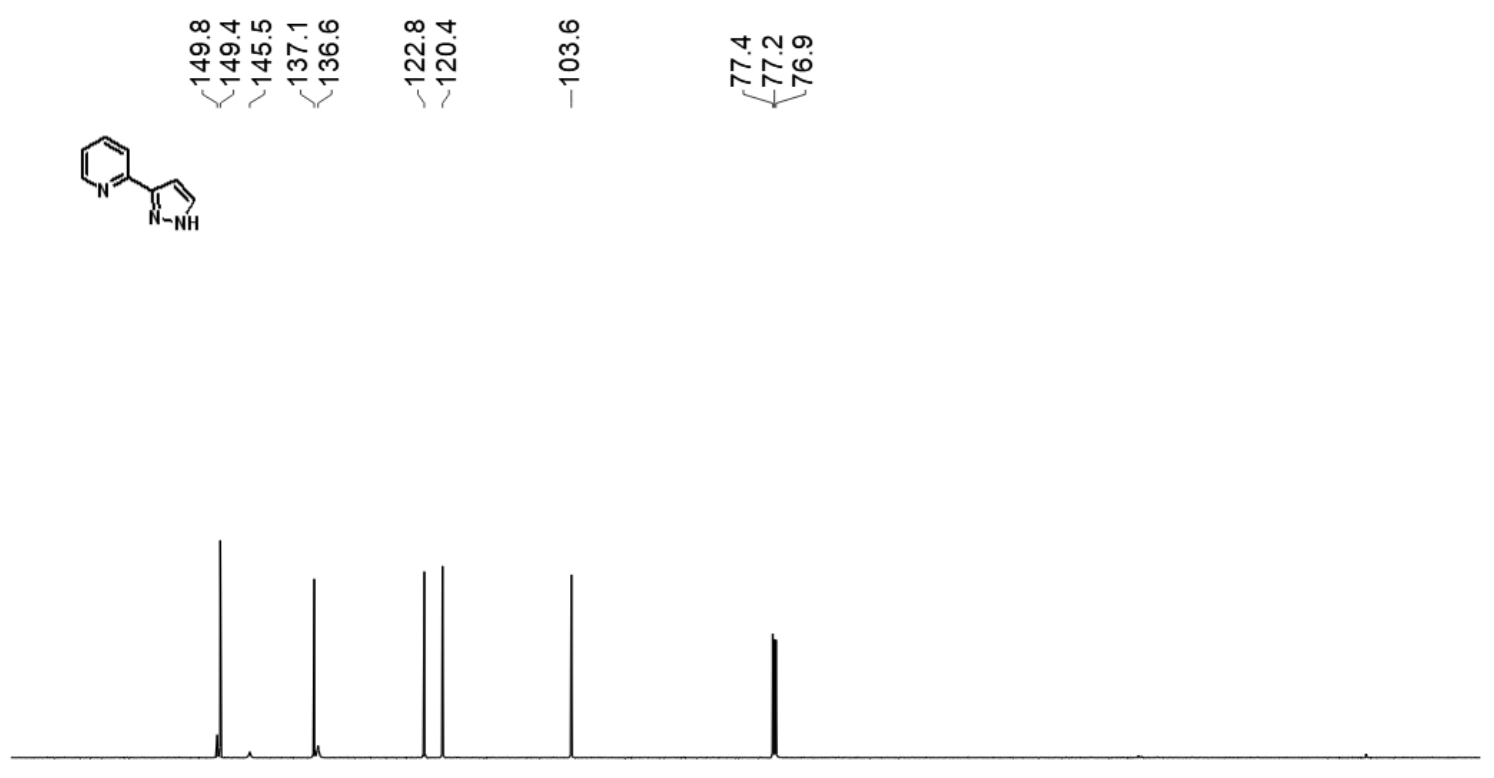

$\begin{array}{lllllllllllllllllll}170 & 160 & 150 & 140 & 130 & 120 & 110 & 100 & 90 & 80 & 70 & 60 & 50 & 40 & 30 & 20 & 10 & 0 & -10\end{array}$

Figure S3. ${ }^{13} \mathrm{C}$ NMR spectrum of $\mathbf{1 a}\left(\mathrm{CDCl}_{3}, 150 \mathrm{MHz}, 23{ }^{\circ} \mathrm{C}\right)$

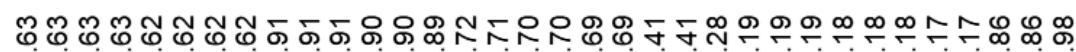

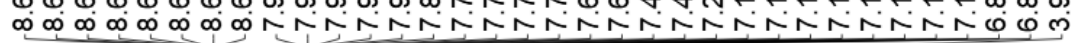<smiles></smiles>

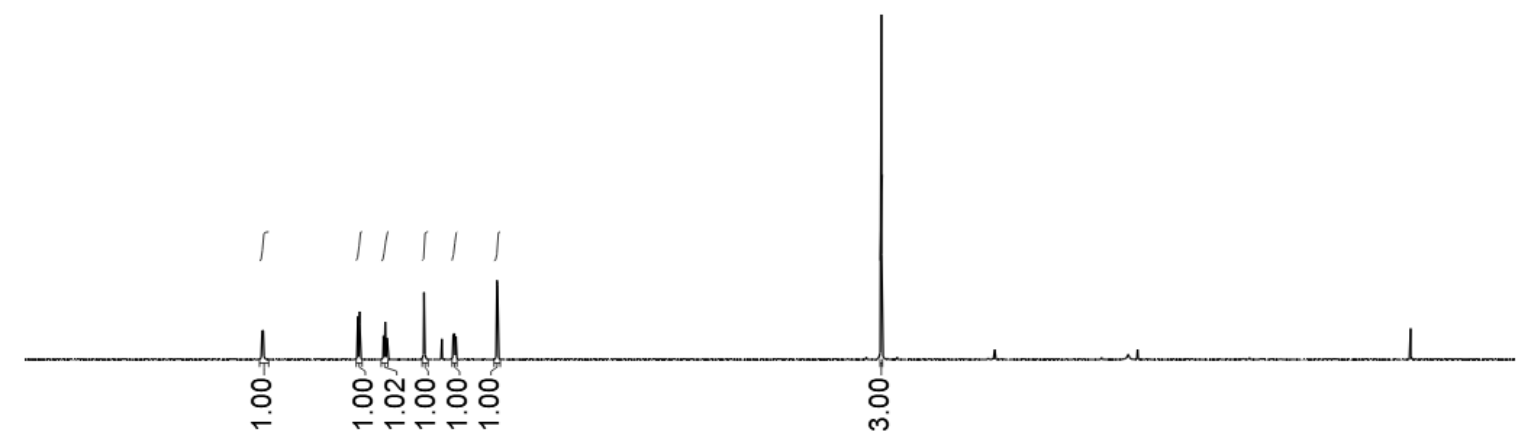

$\begin{array}{lllllllllllllllllllllll}10.0 & 9.5 & 9.0 & 8.5 & 8.0 & 7.5 & 7.0 & 6.5 & 6.0 & 5.5 & \begin{array}{c}5.0 \\ \mathrm{f} 1(\mathrm{ppm})\end{array} & 4.0 & 3.5 & 3.0 & 2.5 & 2.0 & 1.5 & 1.0 & 0.5 & 0.0 & -0.5\end{array}$

Figure S4. ${ }^{1} \mathrm{H} \mathrm{NMR}$ spectrum of $\mathbf{1 b}\left(\mathrm{CDCl}_{3}, 600 \mathrm{MHz}, 23{ }^{\circ} \mathrm{C}\right)$. 


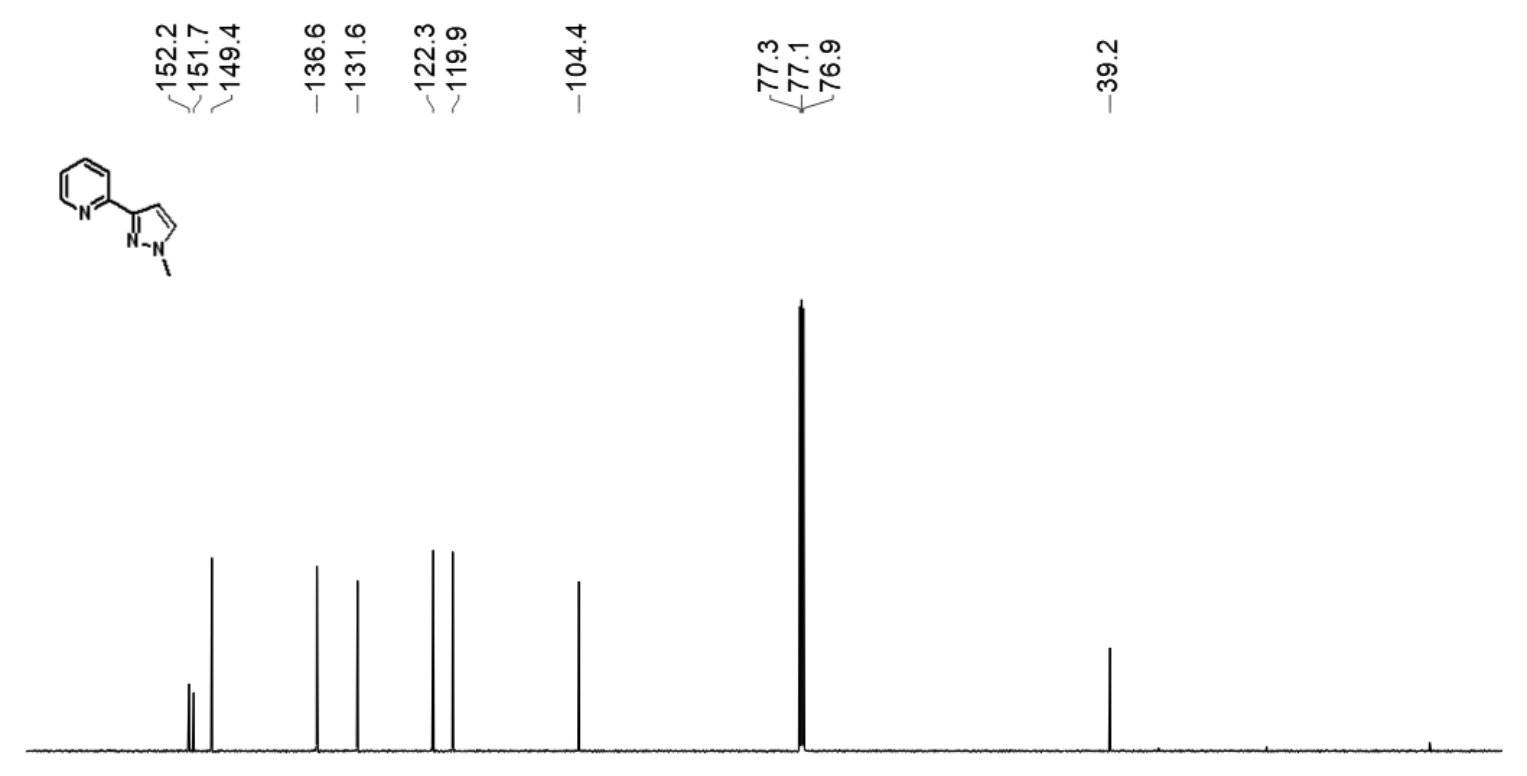

$\begin{array}{lllllllllllllllll}170 & 160 & 150 & 140 & 130 & 120 & 110 & 100 & 90 \underset{\mathrm{f} 1(\mathrm{ppm})}{80} & 70 & 60 & 50 & 40 & 30 & 20 & 10 & 0\end{array}$

Figure S5. ${ }^{13} \mathrm{C}$ NMR spectrum of $\mathbf{1 b}\left(\mathrm{CDCl}_{3}, 150 \mathrm{MHz}, 23{ }^{\circ} \mathrm{C}\right)$.

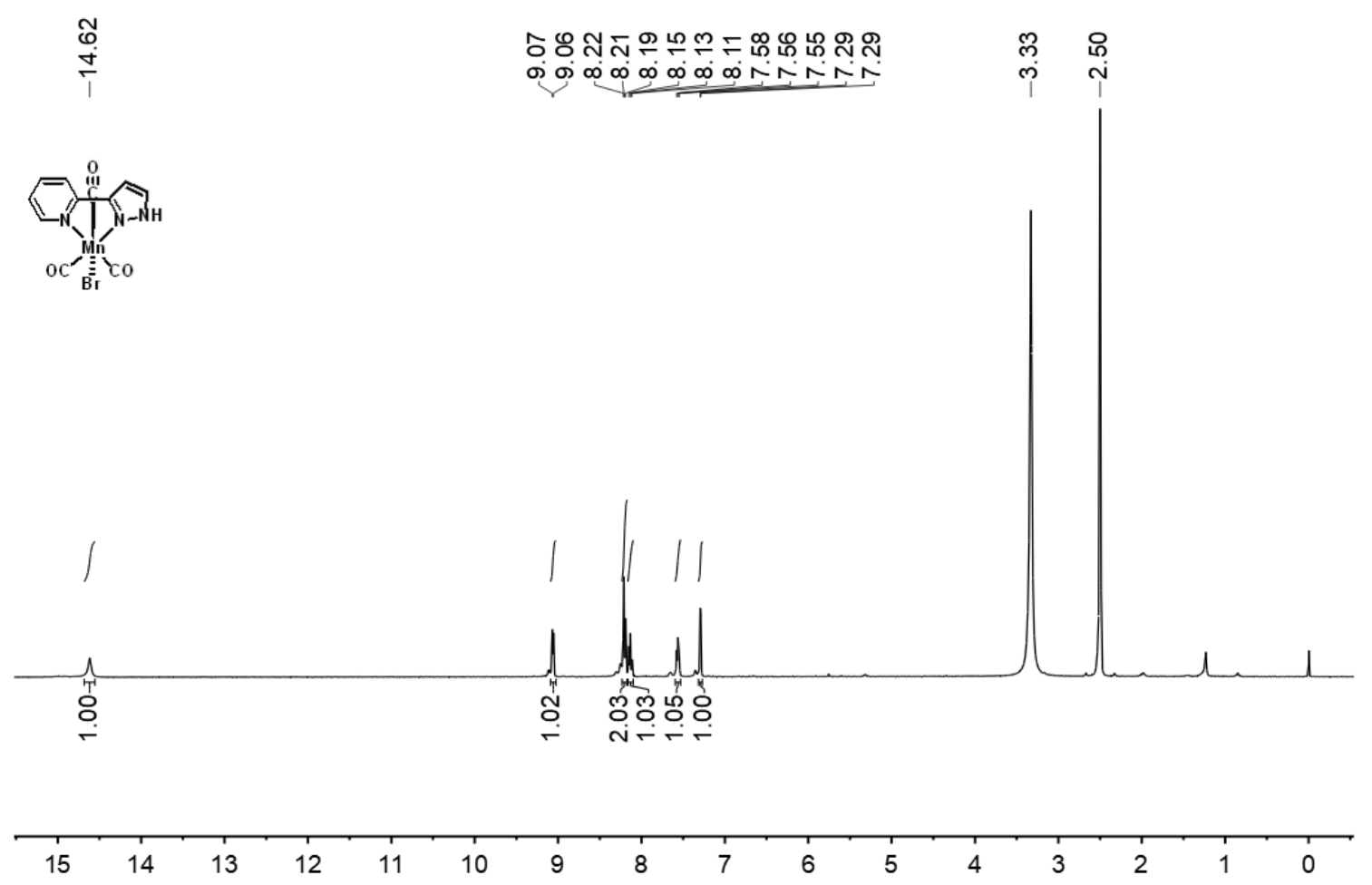

Figure S6. ${ }^{1} \mathrm{H}$ NMR spectrum of $\mathbf{2 a}\left(\mathrm{CD}_{3} \mathrm{SOCD}_{3}, 600 \mathrm{MHz}, 23{ }^{\circ} \mathrm{C}\right)$. 


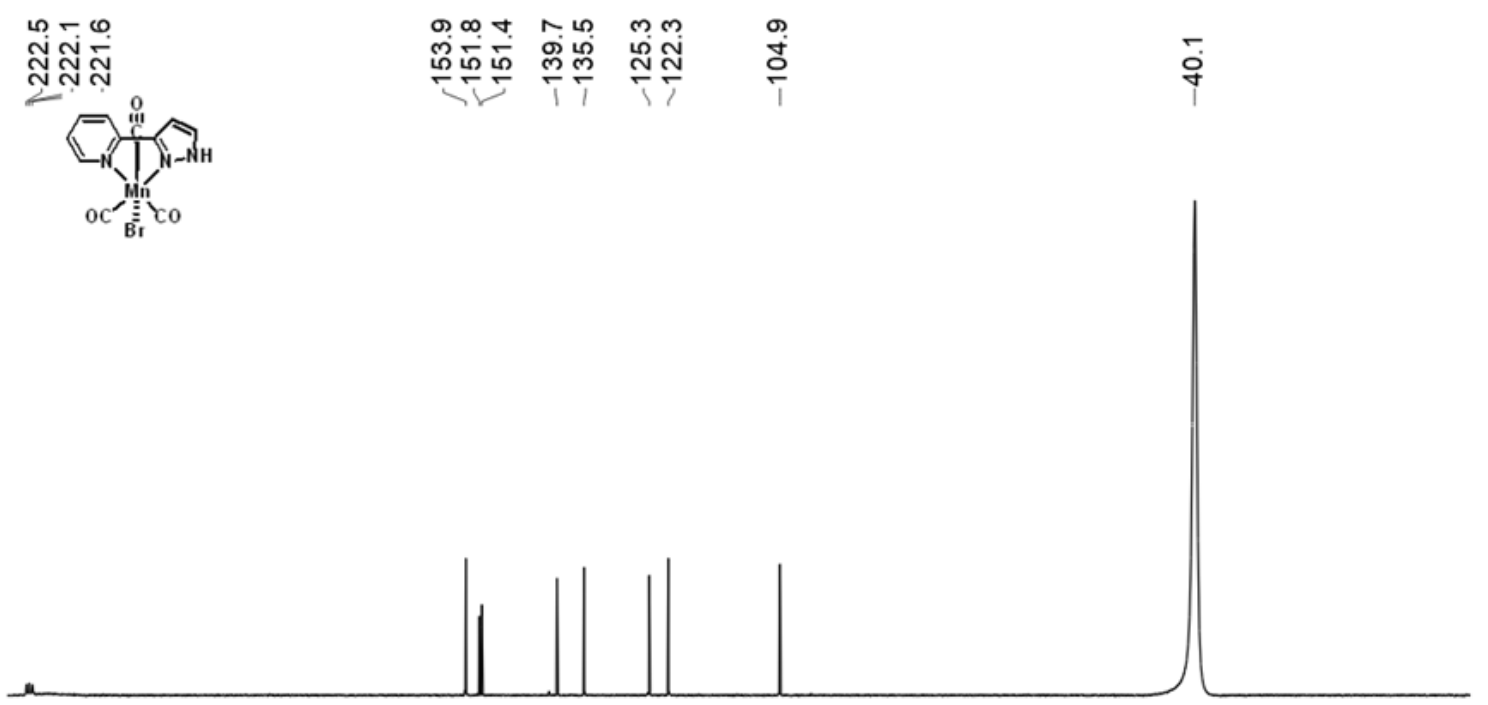

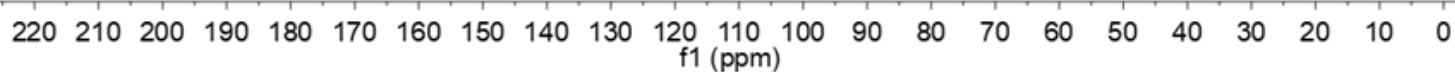

Figure S7. ${ }^{3} \mathrm{C}$ NMR spectrum of $2 \mathbf{a}\left(\mathrm{CD}_{3} \mathrm{SOCD}_{3}, 150 \mathrm{MHz}, 23{ }^{\circ} \mathrm{C}\right)$.
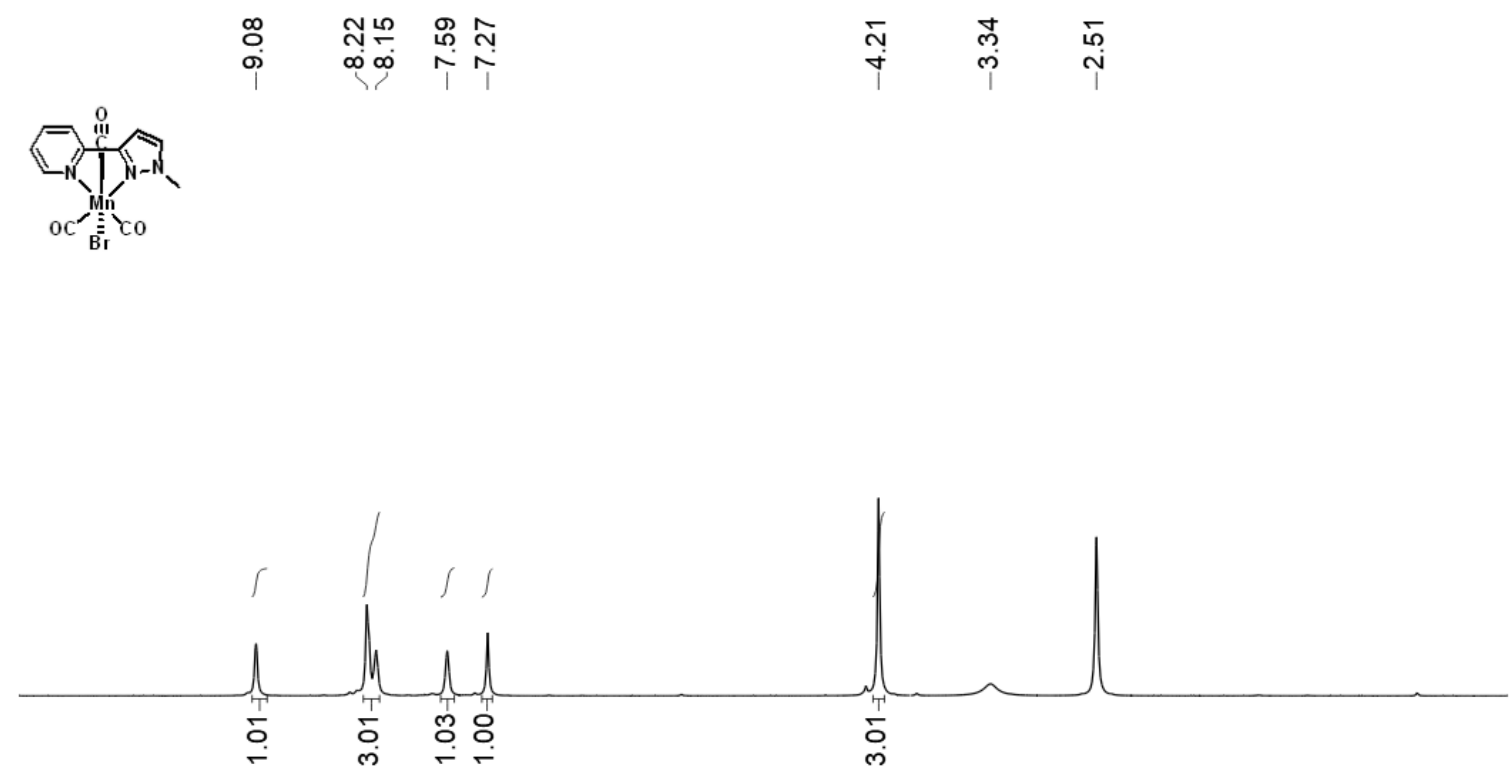

$\begin{array}{lllllllllllllllllllllll}10.5 & 10.0 & 9.5 & 9.0 & 8.5 & 8.0 & 7.5 & 7.0 & 6.5 & 6.0 & \begin{array}{c}5.5 \\ \mathrm{f} 1(\mathrm{ppm})\end{array} \underset{3.0}{(\mathrm{ppm})} & 4.5 & 4.0 & 3.5 & 3.0 & 2.5 & 2.0 & 1.5 & 1.0 & 0.5 & 0.0 & -0.5\end{array}$

Figure S8. ${ }^{1} \mathrm{H}$ NMR spectrum of $\mathbf{2 b}\left(\mathrm{CD}_{3} \mathrm{SOCD}_{3}, 600 \mathrm{MHz}, 23{ }^{\circ} \mathrm{C}\right)$. 


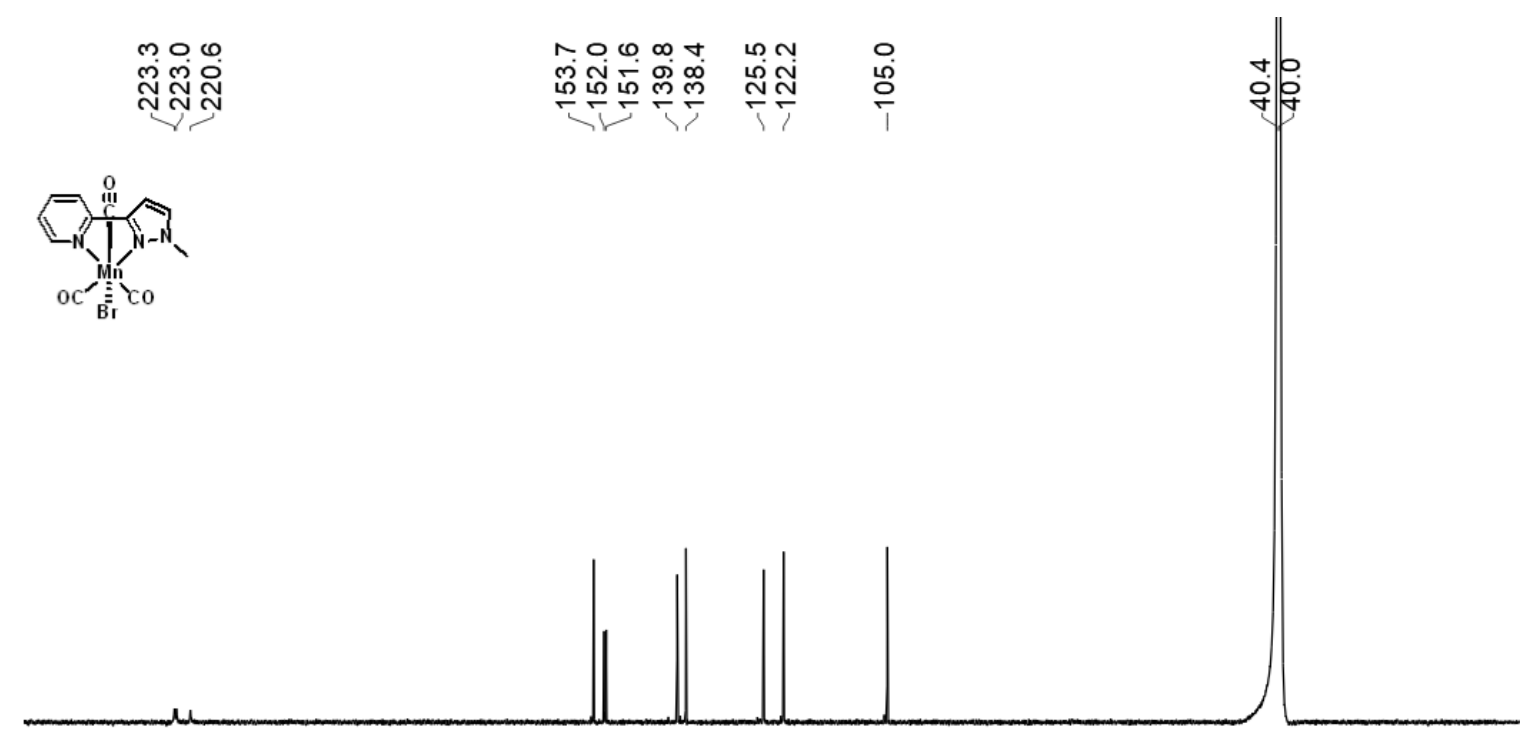

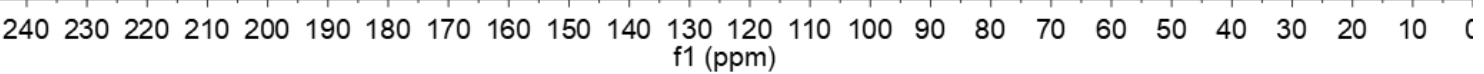

Figure S9. ${ }^{13} \mathrm{C}$ NMR spectrum of $\mathbf{2 b}\left(\mathrm{CD}_{3} \mathrm{SOCD}_{3}, 150 \mathrm{MHz}, 23{ }^{\circ} \mathrm{C}\right)$.
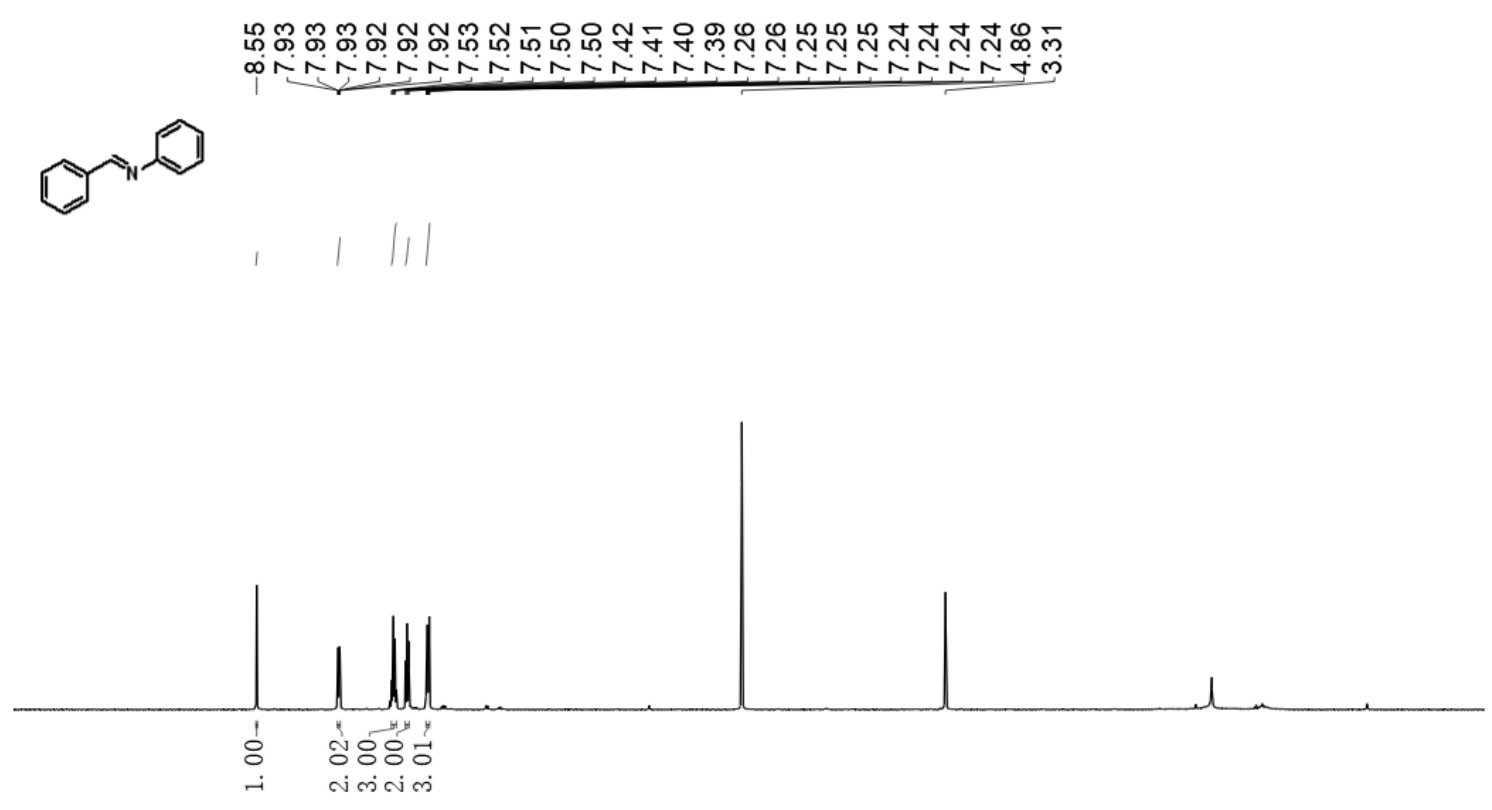

$\begin{array}{llllllllllllllllllllll}10.0 & 9.5 & 9.0 & 8.5 & 8.0 & 7.5 & 7.0 & 6.5 & 6.0 & 5.5 & \begin{array}{c}5.0 \\ \mathrm{f} 1(\mathrm{ppm})\end{array} & 4.0 & 3.5 & 3.0 & 2.5 & 2.0 & 1.5 & 1.0 & 0.5 & 0.0 & -0.5\end{array}$

Figure S10. ${ }^{1} \mathrm{H}$ NMR spectrum of $\mathbf{5 a}\left(\mathrm{CD}_{3} \mathrm{OD}, 600 \mathrm{MHz}, 23^{\circ} \mathrm{C}\right)$. 


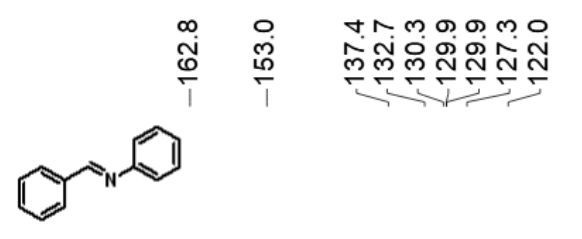

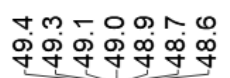

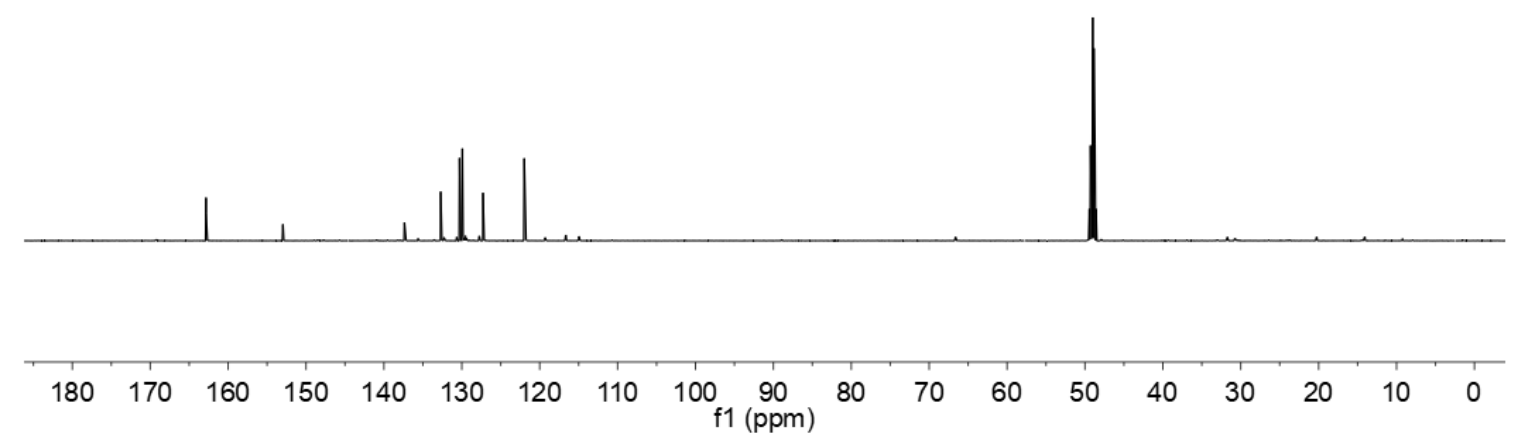

Figure S11. ${ }^{13} \mathrm{C}$ NMR spectrum of $\mathbf{5 a}\left(\mathrm{CD}_{3} \mathrm{OD}, 150 \mathrm{MHz}, 23{ }^{\circ} \mathrm{C}\right)$.

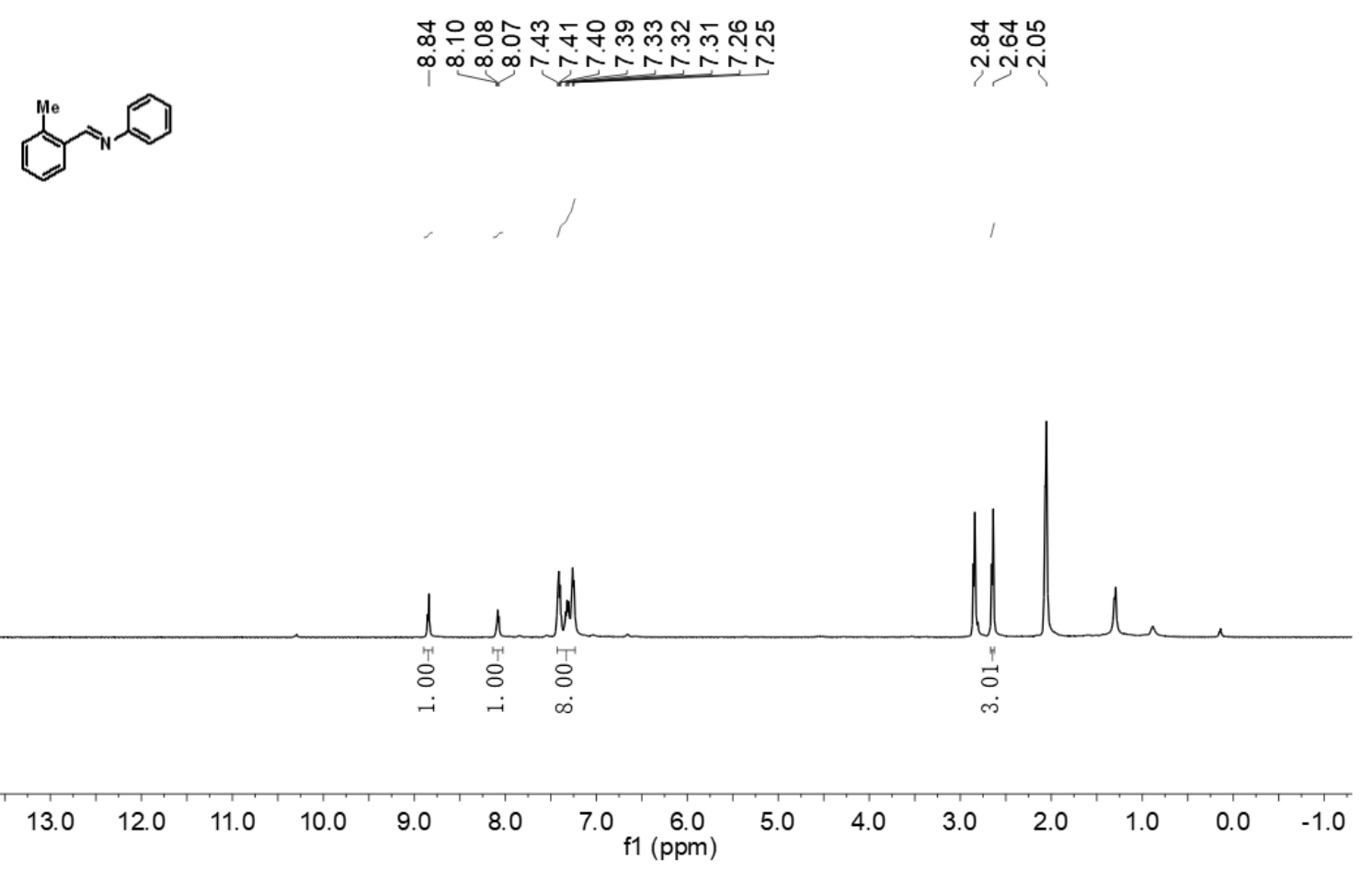

Figure S12. ${ }^{1} \mathrm{H}$ NMR spectrum of $\mathbf{5 b}\left(\mathrm{CD}_{3} \mathrm{COCD}_{3}, 600 \mathrm{MHz}, 23{ }^{\circ} \mathrm{C}\right)$. 


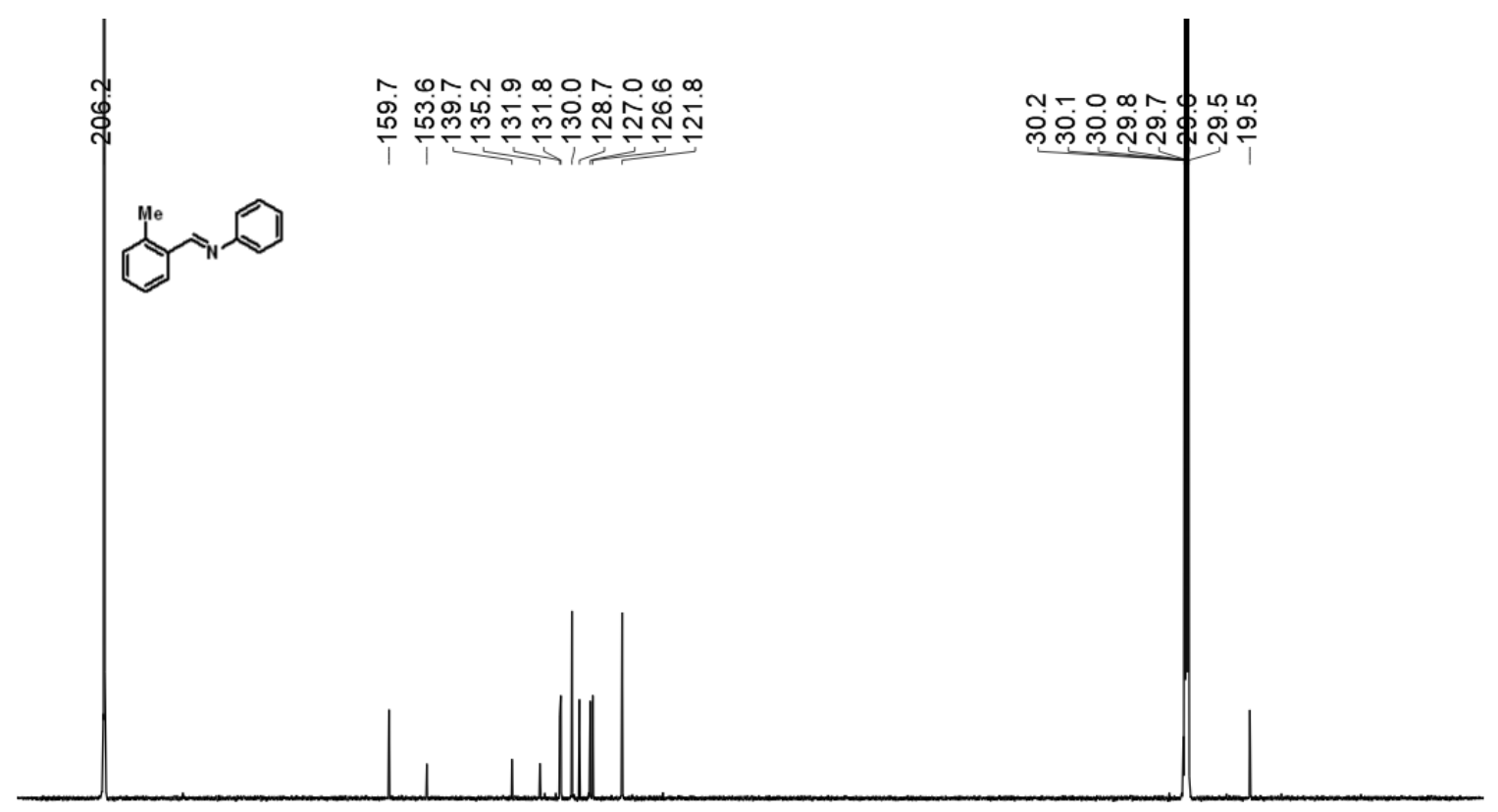

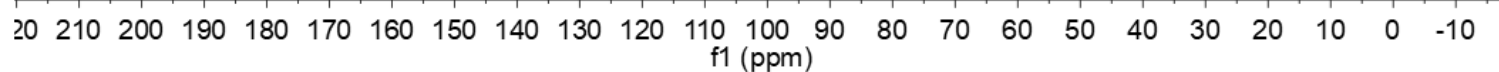

Figure S13. ${ }^{13} \mathrm{C}$ NMR spectrum of $\mathbf{5 b}\left(\mathrm{CD}_{3} \mathrm{COCD}_{3}, 150 \mathrm{MHz}, 23{ }^{\circ} \mathrm{C}\right)$.

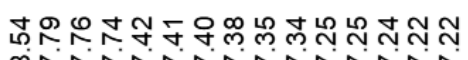

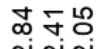

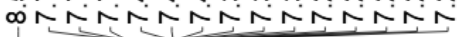

i่ง

Men

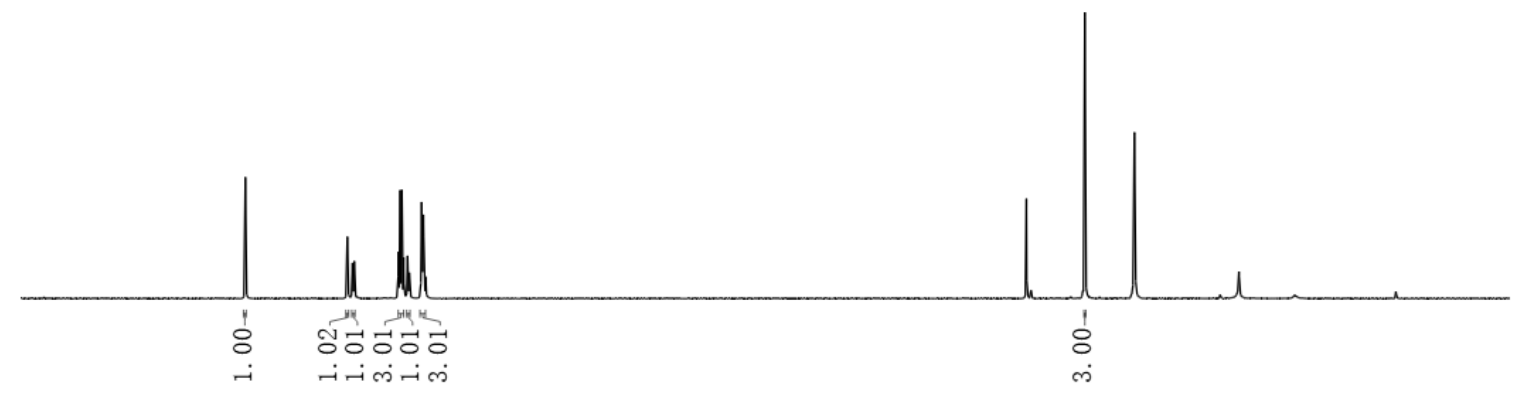

$\begin{array}{llllllllllllllllllllll}10.0 & 9.5 & 9.0 & 8.5 & 8.0 & 7.5 & 7.0 & 6.5 & 6.0 & 5.5 & \begin{array}{r}5.0 \\ \mathrm{f} 1(\mathrm{ppm})\end{array} & 4.5 & 4.0 & 3.5 & 3.0 & 2.5 & 2.0 & 1.5 & 1.0 & 0.5 & 0.0 & -0.5\end{array}$

Figure S14. ${ }^{1} \mathrm{H}$ NMR spectrum of $\mathbf{5 c}\left(\mathrm{CD}_{3} \mathrm{COCD}_{3}, 600 \mathrm{MHz}, 23^{\circ} \mathrm{C}\right)$. 

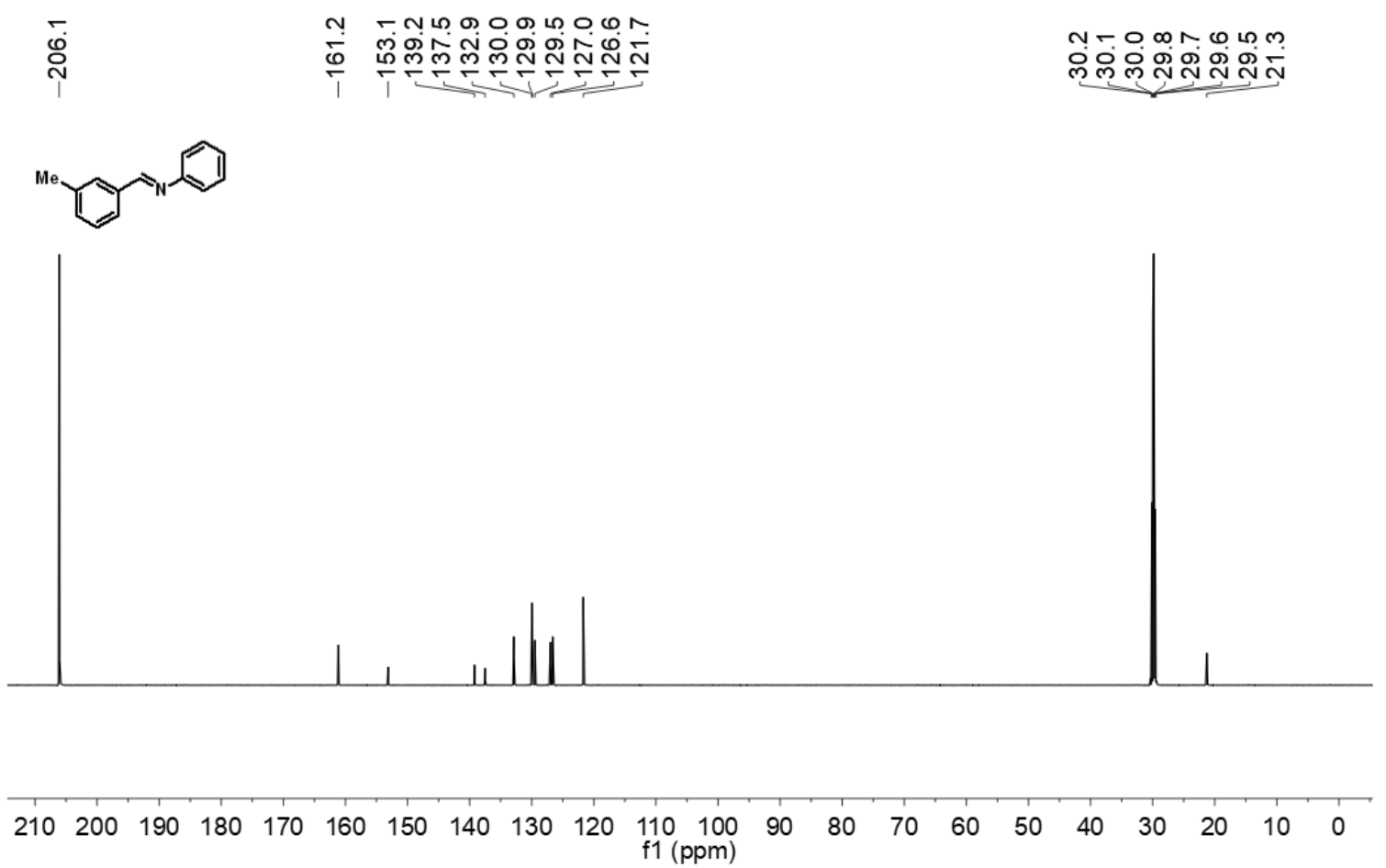

Figure S15. ${ }^{13} \mathrm{C}$ NMR spectrum of $\mathbf{5 c}\left(\mathrm{CD}_{3} \mathrm{COCD}_{3}, 150 \mathrm{MHz}, 23{ }^{\circ} \mathrm{C}\right)$.
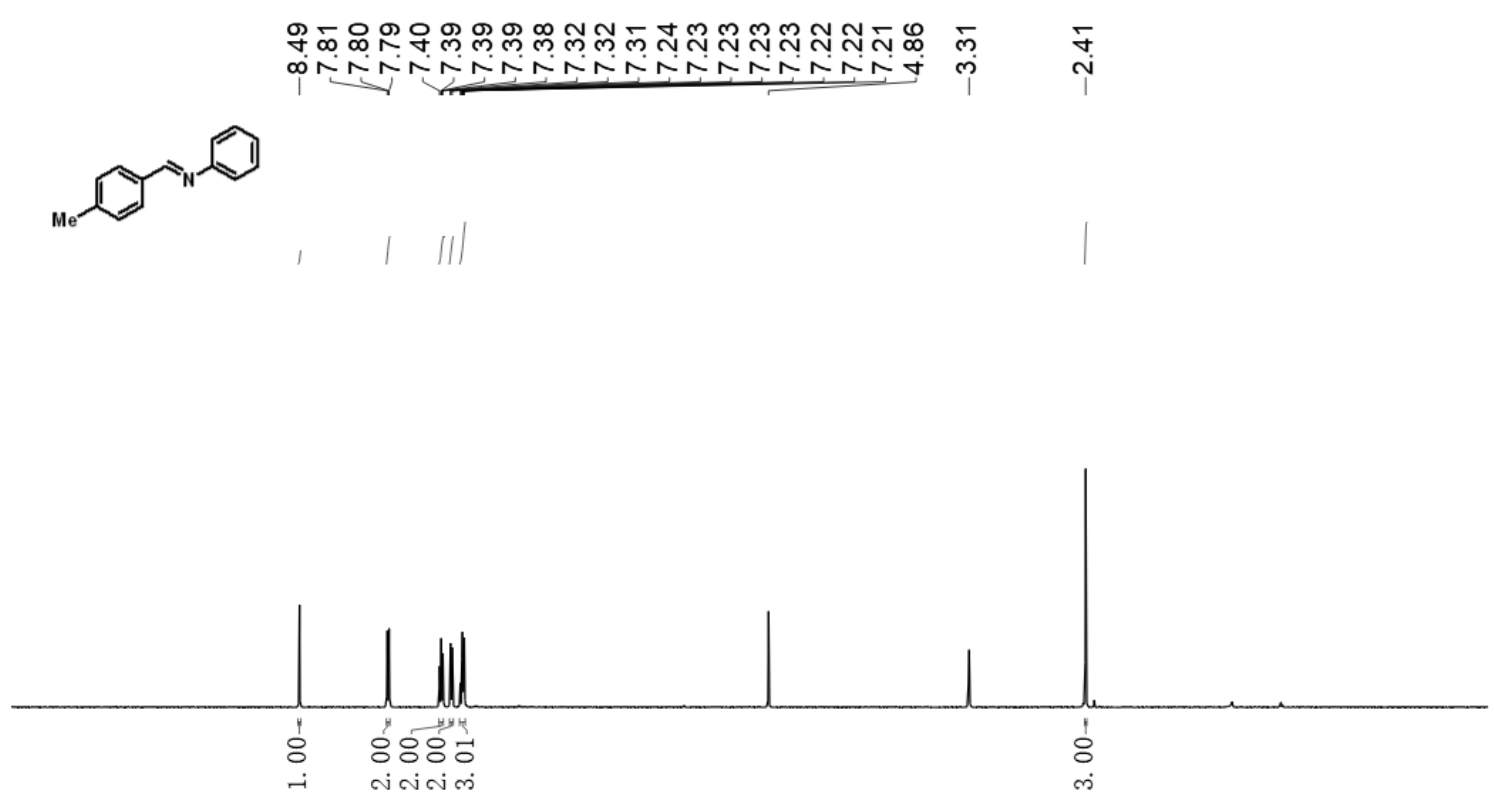

$\begin{array}{lllllllllllllllllllllllllllllll}10.5 & 10.0 & 9.5 & 9.0 & 8.5 & 8.0 & 7.5 & 7.0 & 6.5 & 6.0 & 5.5 & \begin{array}{c}5.0 \\ f 1\end{array}(\mathrm{ppm}) & 4.5 & 4.0 & 3.5 & 3.0 & 2.5 & 2.0 & 1.5 & 1.0 & 0.5 & 0.0 & -0.5\end{array}$

Figure S16. ${ }^{1} \mathrm{H}$ NMR spectrum of $\mathbf{5 d}\left(\mathrm{CD}_{3} \mathrm{OD}, 600 \mathrm{MHz}, 23^{\circ} \mathrm{C}\right)$. 

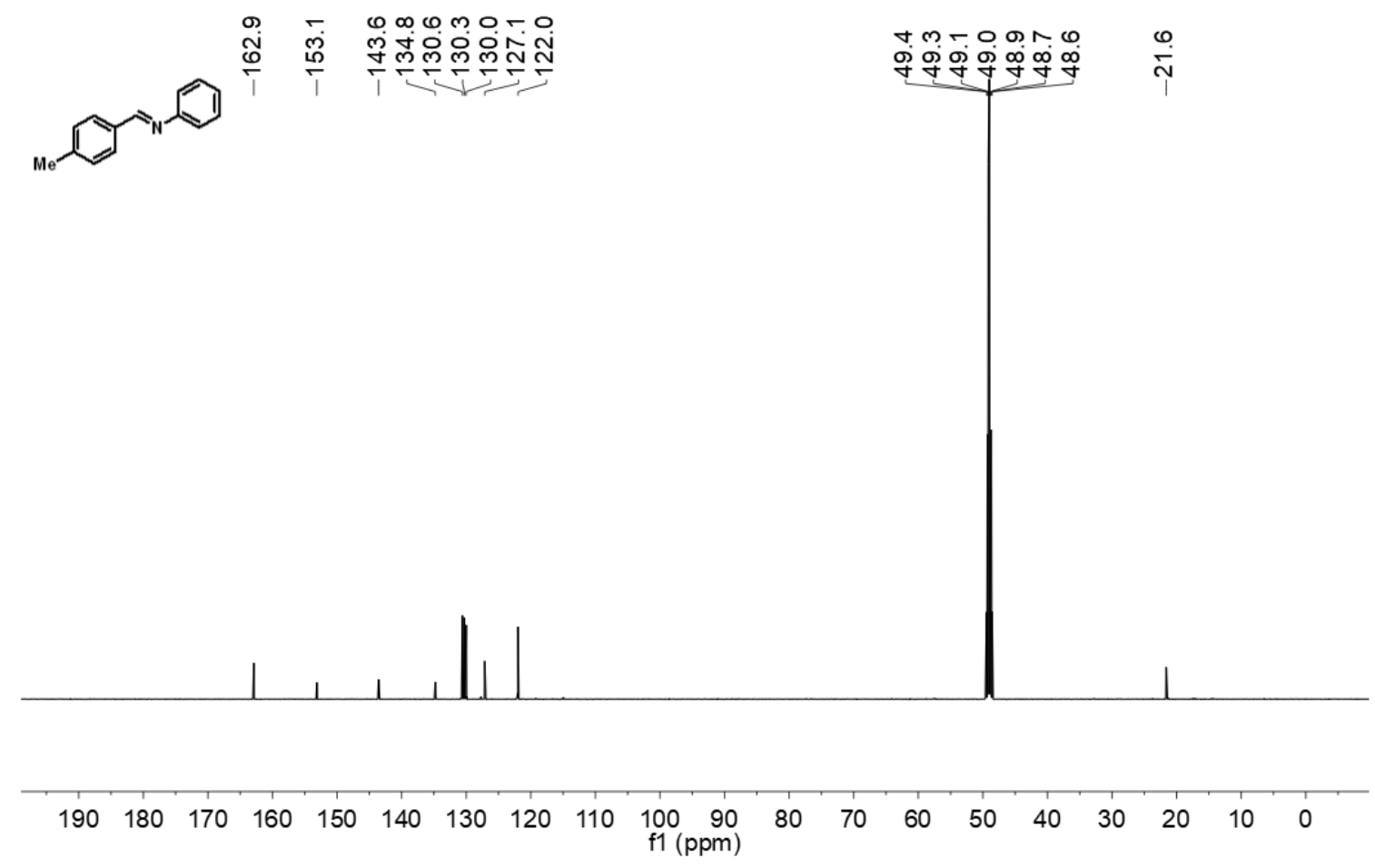

Figure S17. ${ }^{13} \mathrm{C}$ NMR spectrum of $\mathbf{5 d}\left(\mathrm{CD}_{3} \mathrm{OD}, 150 \mathrm{MHz}, 23{ }^{\circ} \mathrm{C}\right)$.

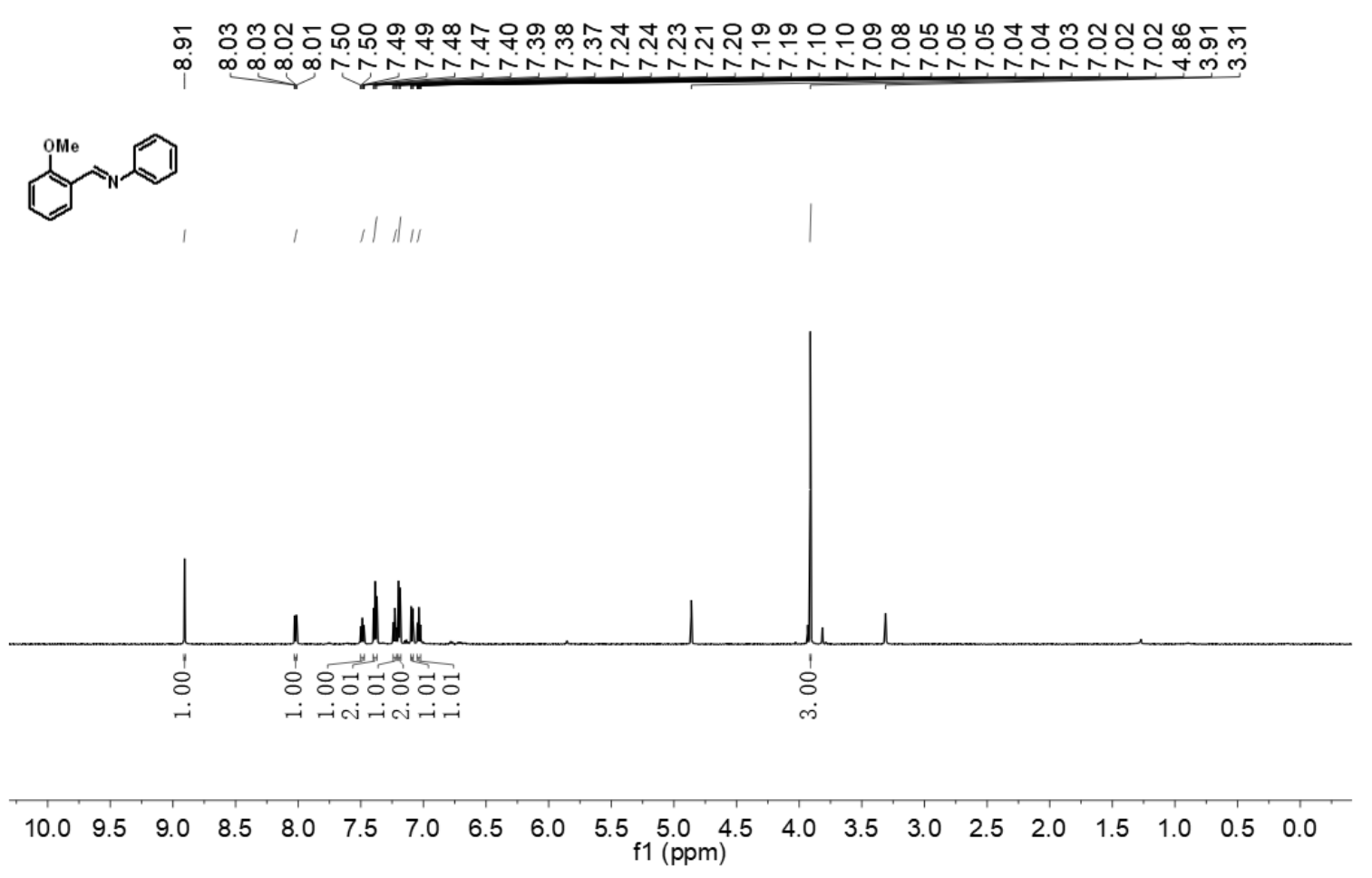

Figure S18. ${ }^{1} \mathrm{H}$ NMR spectrum of $\mathbf{5 e}\left(\mathrm{CD}_{3} \mathrm{OD}, 600 \mathrm{MHz}, 23{ }^{\circ} \mathrm{C}\right)$. 


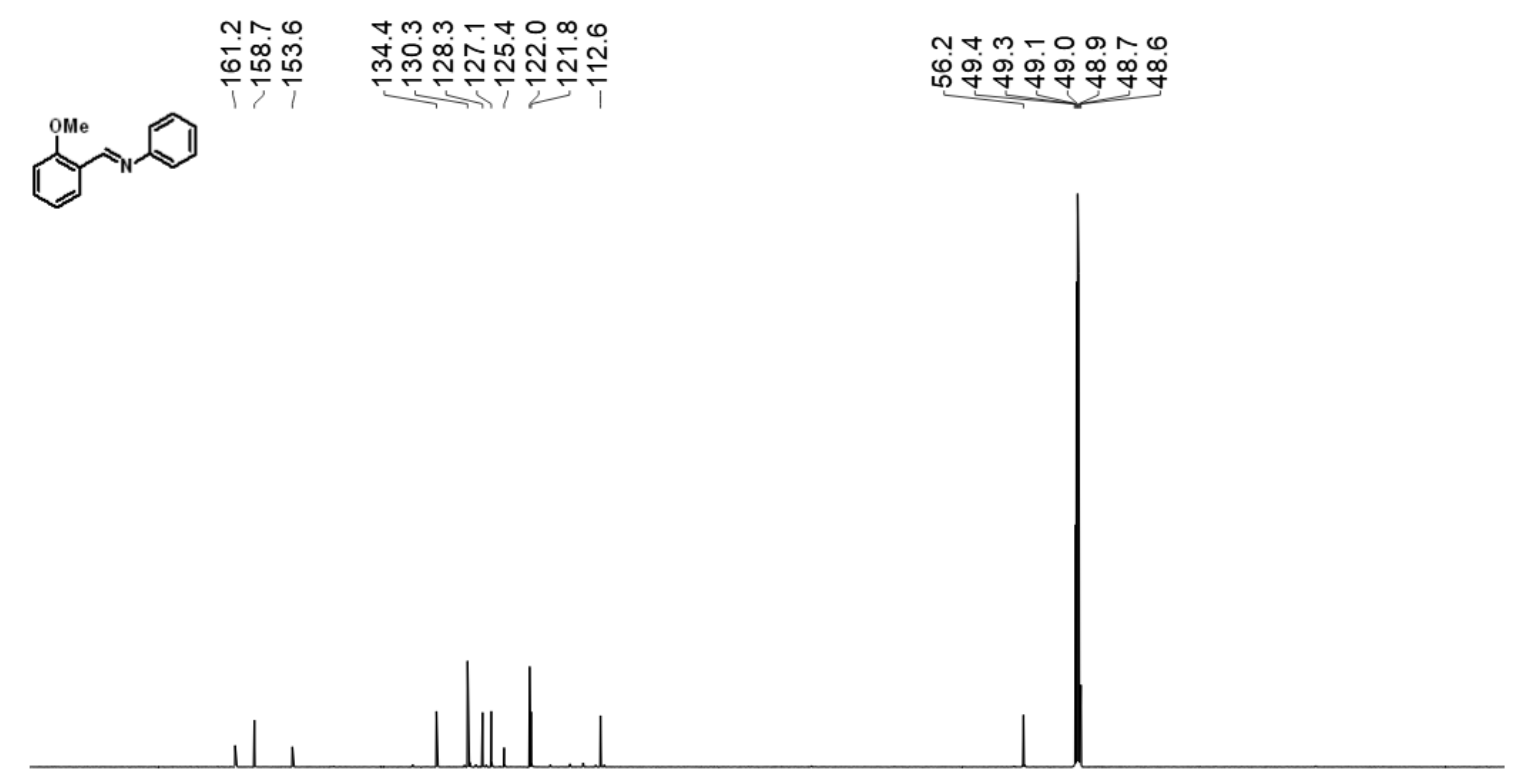

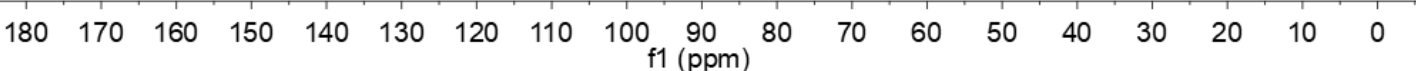

Figure S19. ${ }^{13} \mathrm{C}$ NMR spectrum of $\mathbf{5 e}\left(\mathrm{CD}_{3} \mathrm{OD}, 150 \mathrm{MHz}, 23{ }^{\circ} \mathrm{C}\right)$.

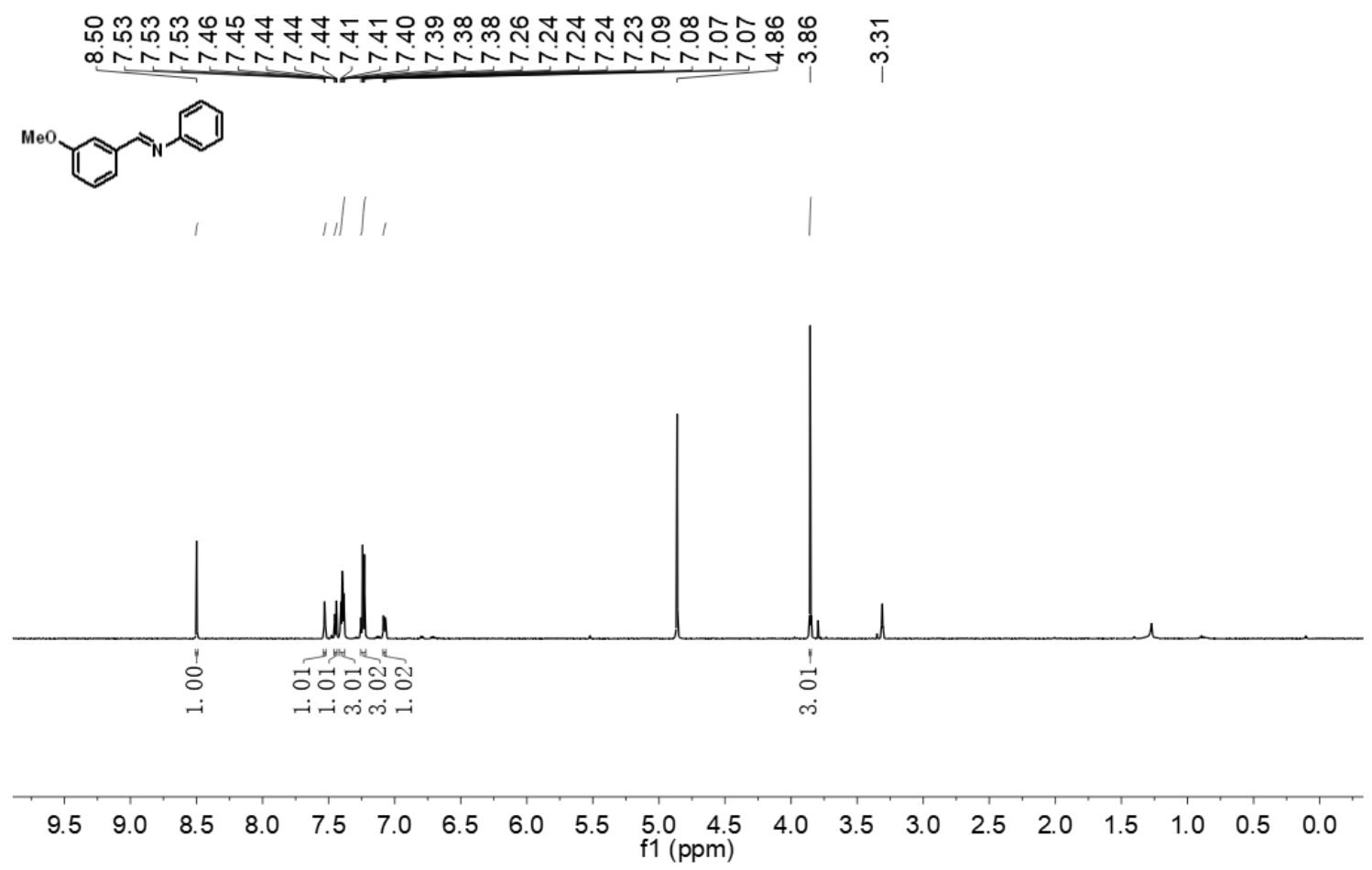

Figure S20. ${ }^{1} \mathrm{H}$ NMR spectrum of $\mathbf{5 f}\left(\mathrm{CD}_{3} \mathrm{OD}, 600 \mathrm{MHz}, 23{ }^{\circ} \mathrm{C}\right)$. 


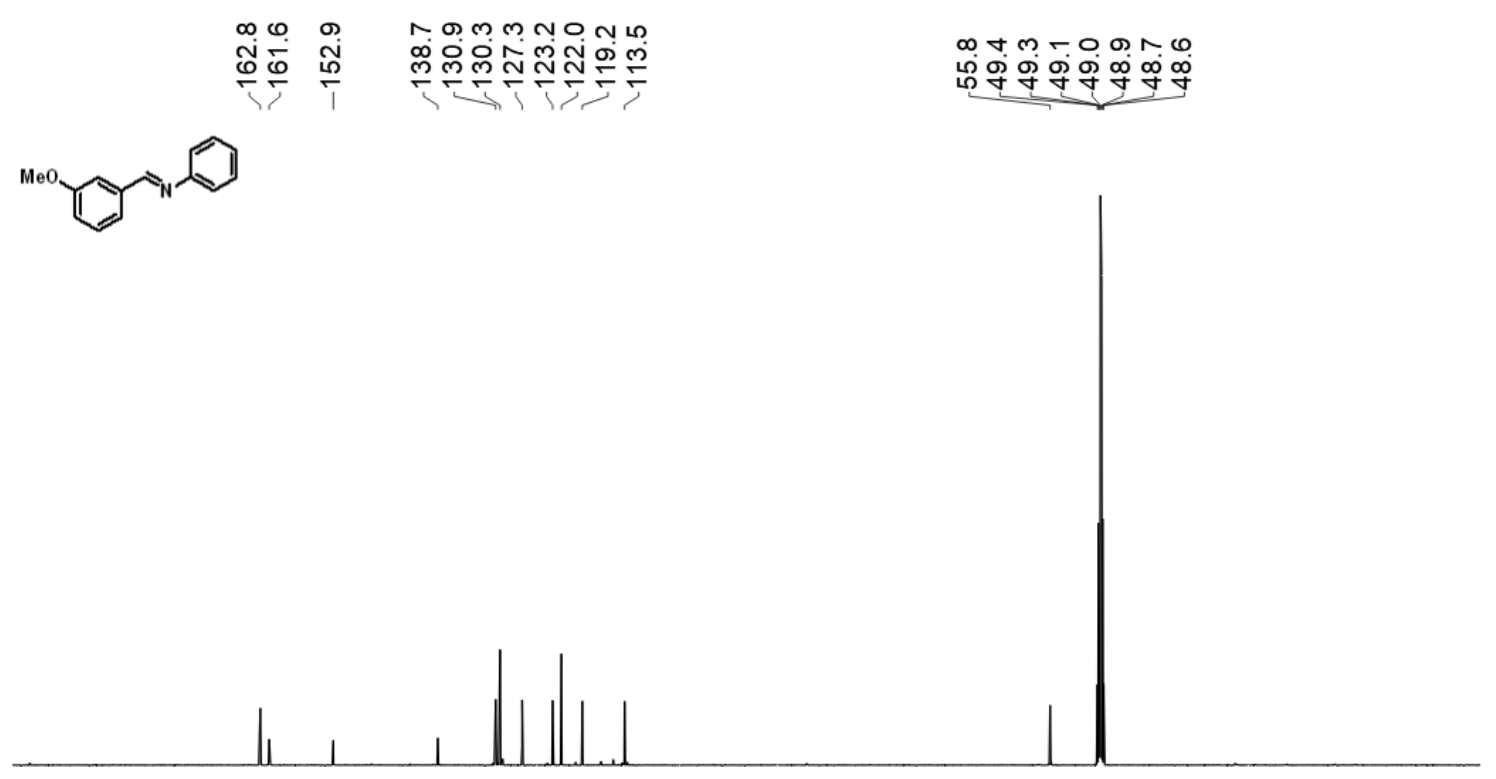

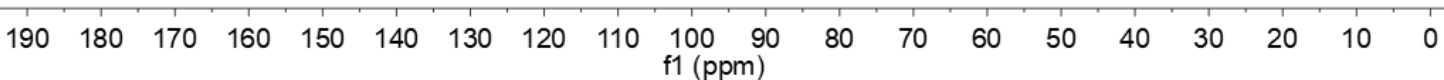

Figure S21. ${ }^{13} \mathrm{C}$ NMR spectrum of $\mathbf{5 f}\left(\mathrm{CD}_{3} \mathrm{OD}, 150 \mathrm{MHz}, 23{ }^{\circ} \mathrm{C}\right)$.

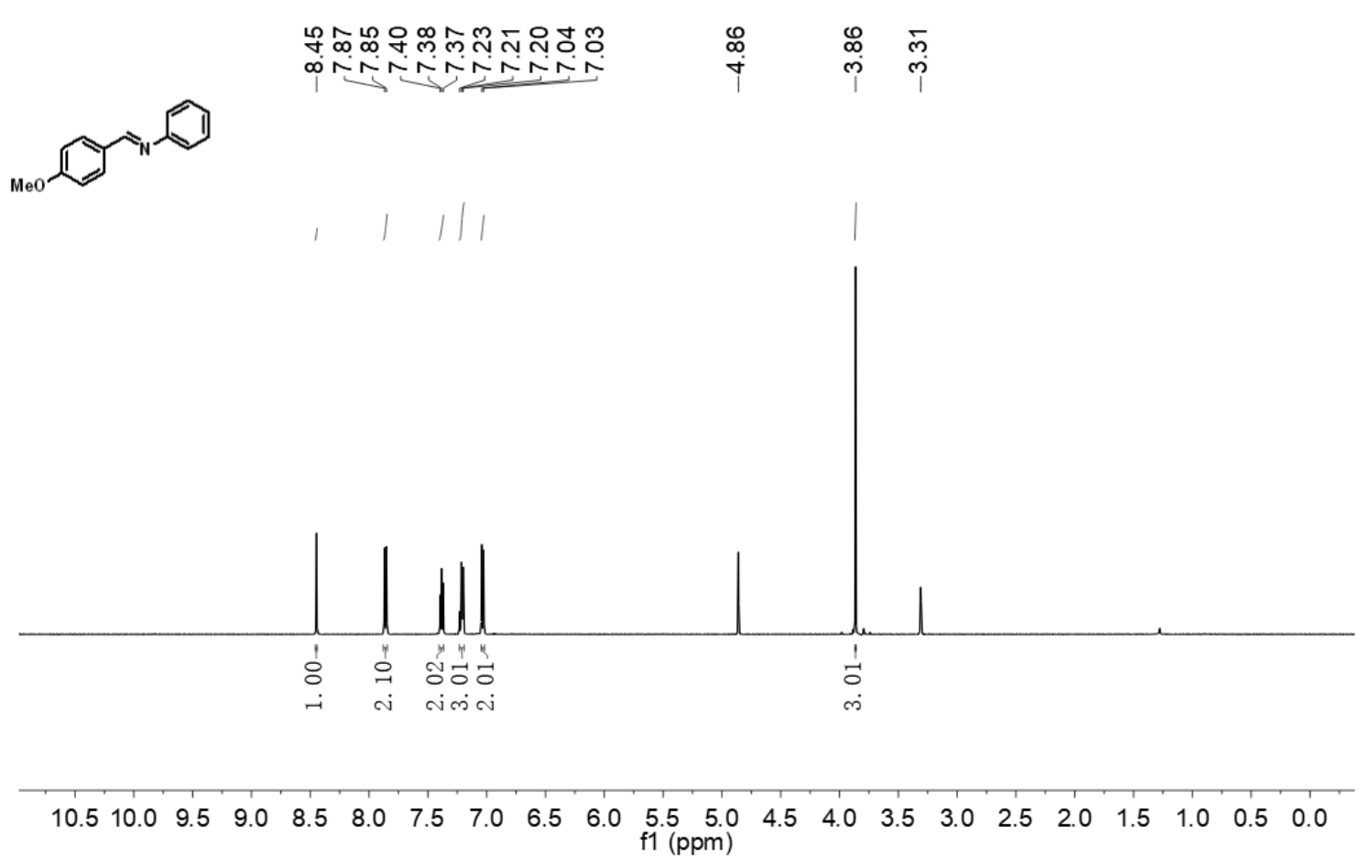

Figure S22. ${ }^{1} \mathrm{H}$ NMR spectrum of $\mathbf{5 g}\left(\mathrm{CD}_{3} \mathrm{OD}, 600 \mathrm{MHz}, 23{ }^{\circ} \mathrm{C}\right)$. 


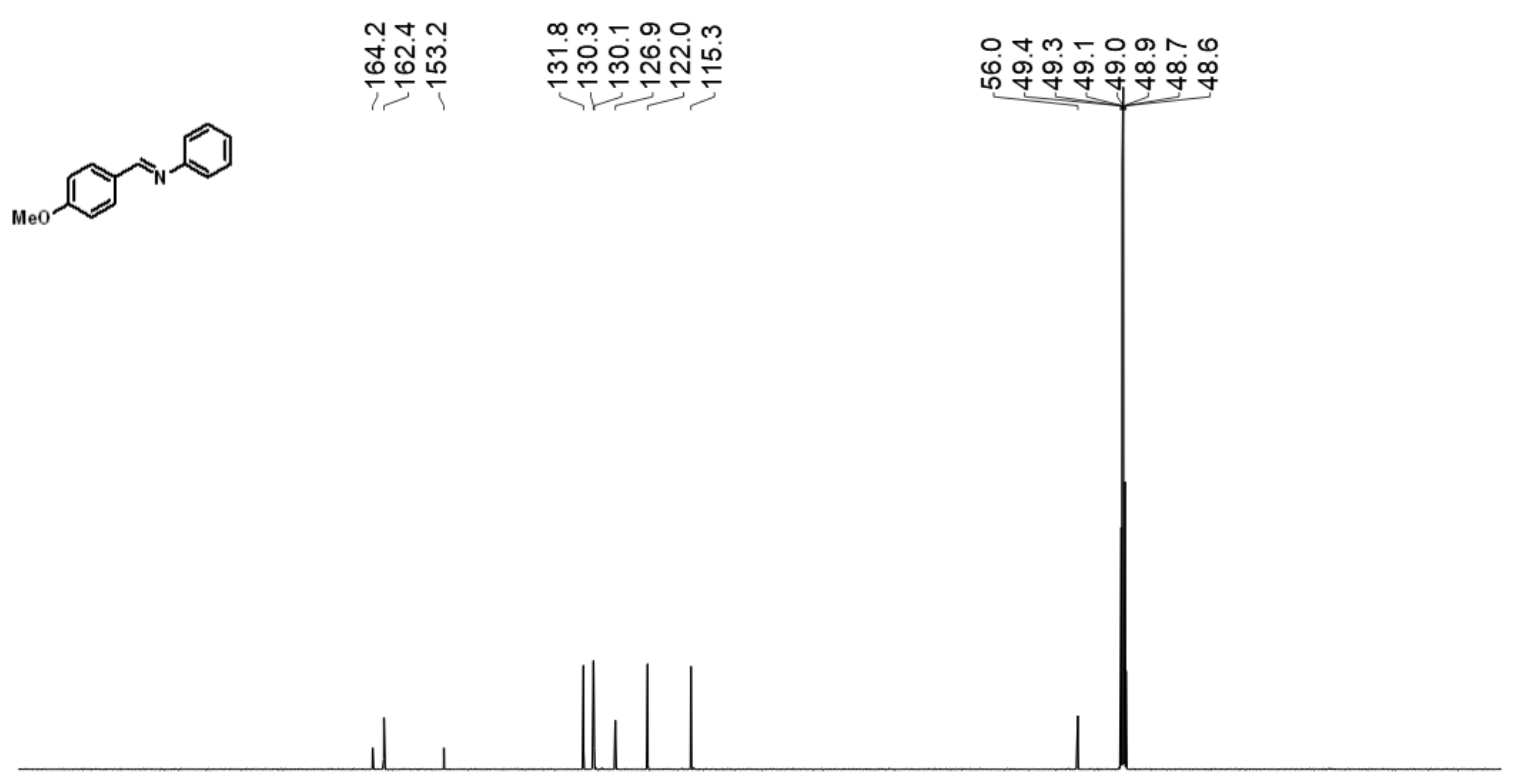

$\begin{array}{lllllllllllllllllllll}210 & 200 & 190 & 180 & 170 & 160 & 150 & 140 & 130 & 120 & \begin{array}{l}110 \\ \mathrm{f} 1(\mathrm{ppm})\end{array} & 100 & 80 & 70 & 60 & 50 & 40 & 30 & 20 & 10 & 0\end{array}$

Figure S23. ${ }^{13} \mathrm{C}$ NMR spectrum of $\mathbf{5 g}\left(\mathrm{CD}_{3} \mathrm{OD}, 150 \mathrm{MHz}, 23^{\circ} \mathrm{C}\right)$.

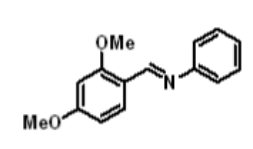

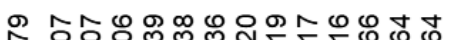

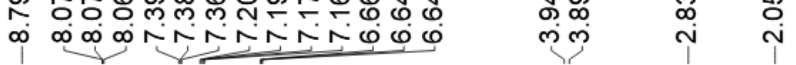

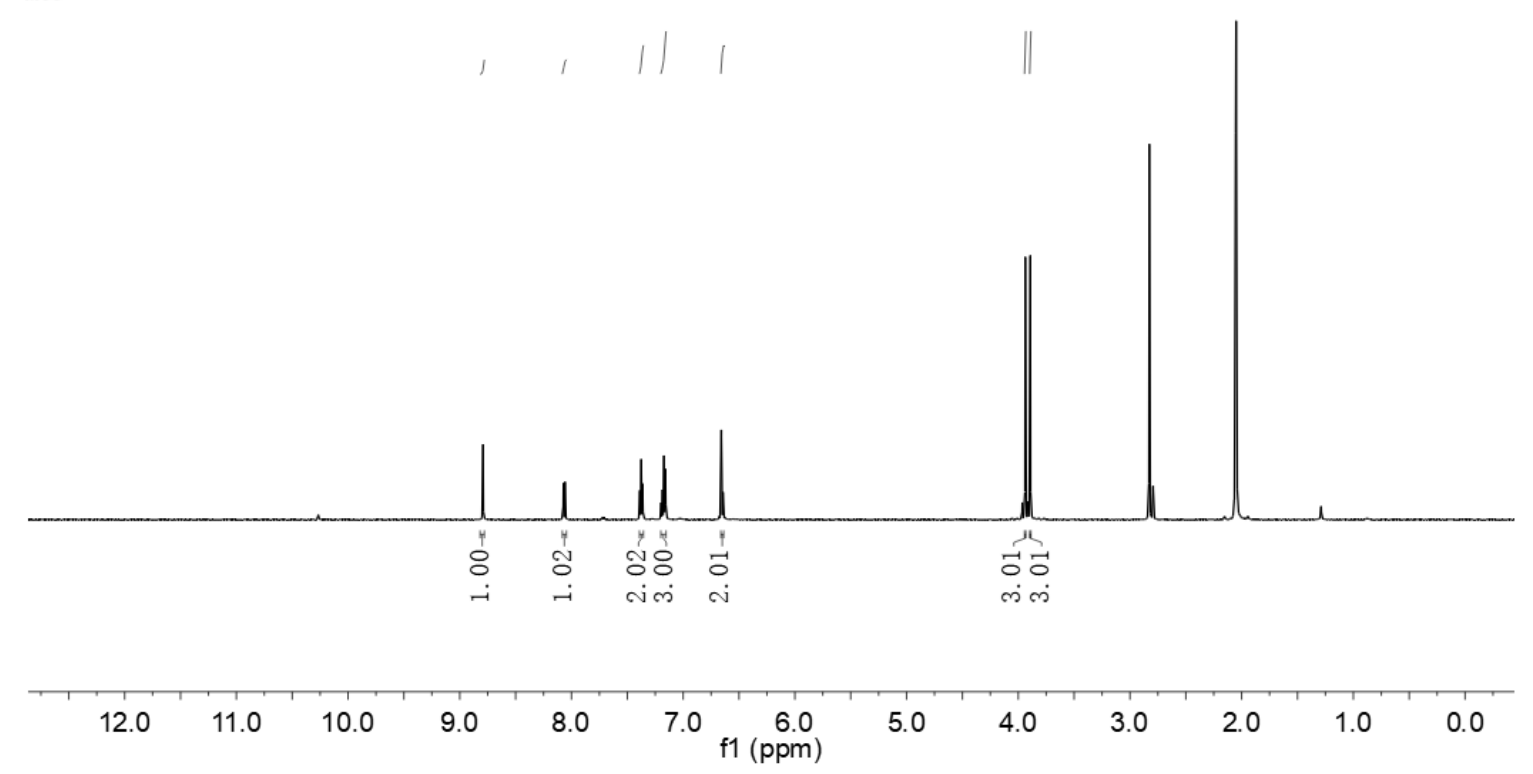

Figure S24. ${ }^{1} \mathrm{H}$ NMR spectrum of $\mathbf{5 h}\left(\mathrm{CD}_{3} \mathrm{COCD}_{3}, 600 \mathrm{MHz}, 23{ }^{\circ} \mathrm{C}\right)$. 


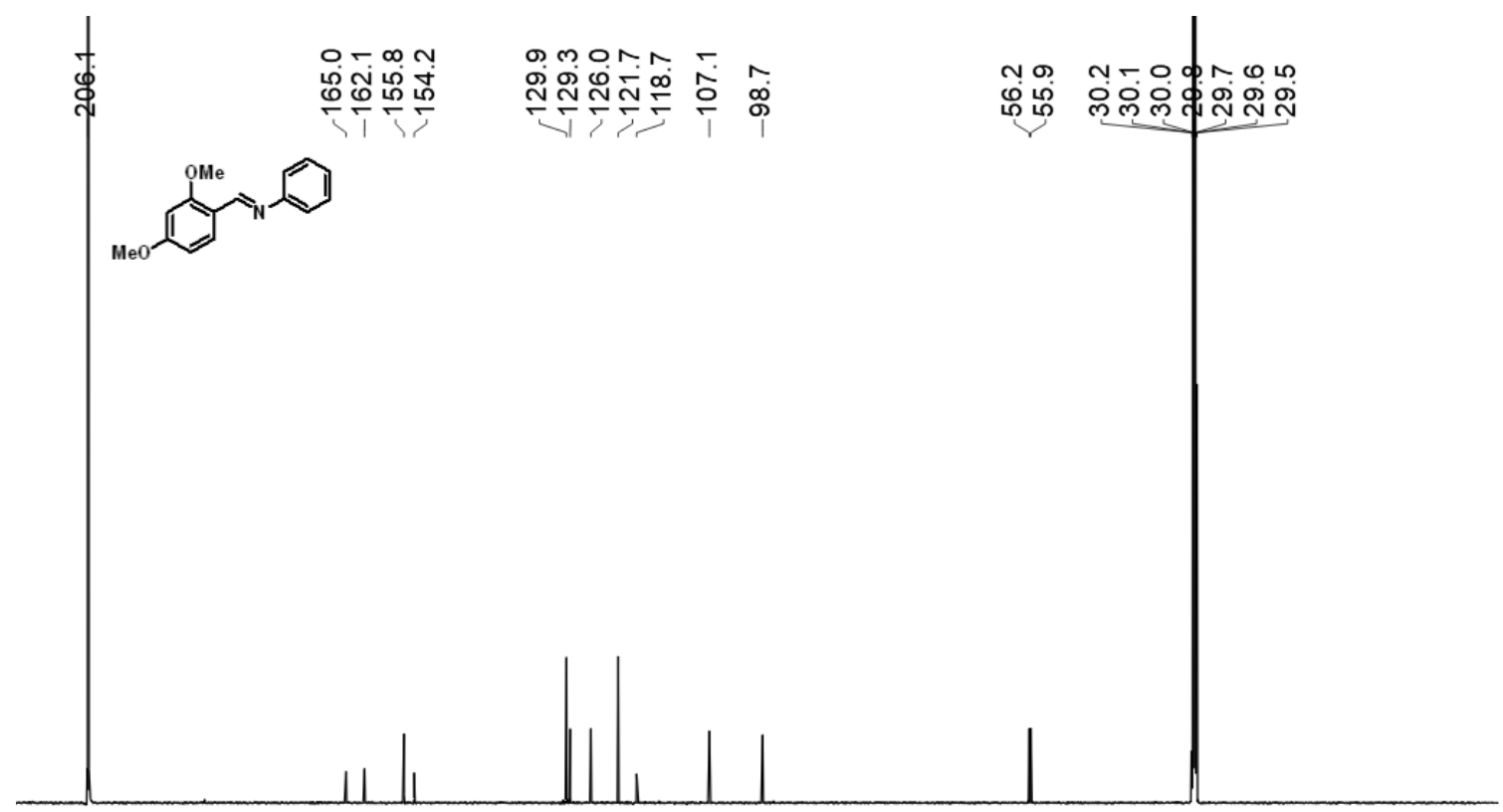

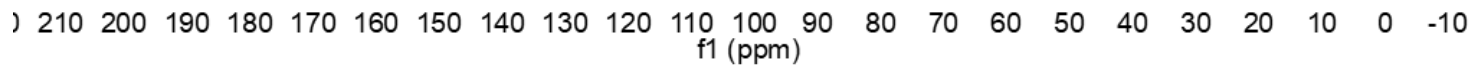

Figure S25. ${ }^{13} \mathrm{C}$ NMR spectrum of $\mathbf{5 h}\left(\mathrm{CD}_{3} \mathrm{COCD}_{3}, 150 \mathrm{MHz}, 23{ }^{\circ} \mathrm{C}\right)$.

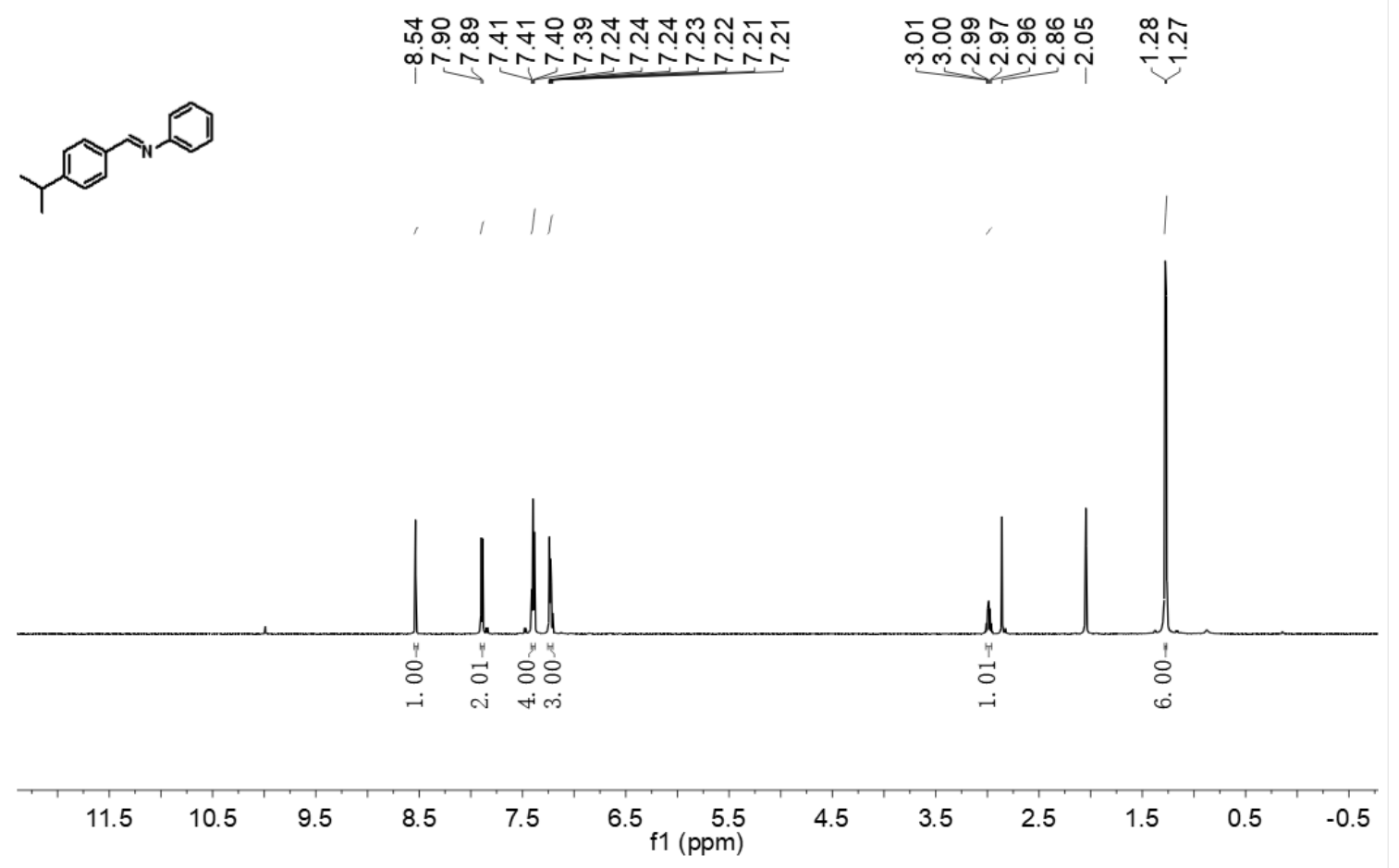

Figure S26. ${ }^{1} \mathrm{H}$ NMR spectrum of $\mathbf{5 i}\left(\mathrm{CD}_{3} \mathrm{COCD}_{3}, 600 \mathrm{MHz}, 23{ }^{\circ} \mathrm{C}\right)$. 


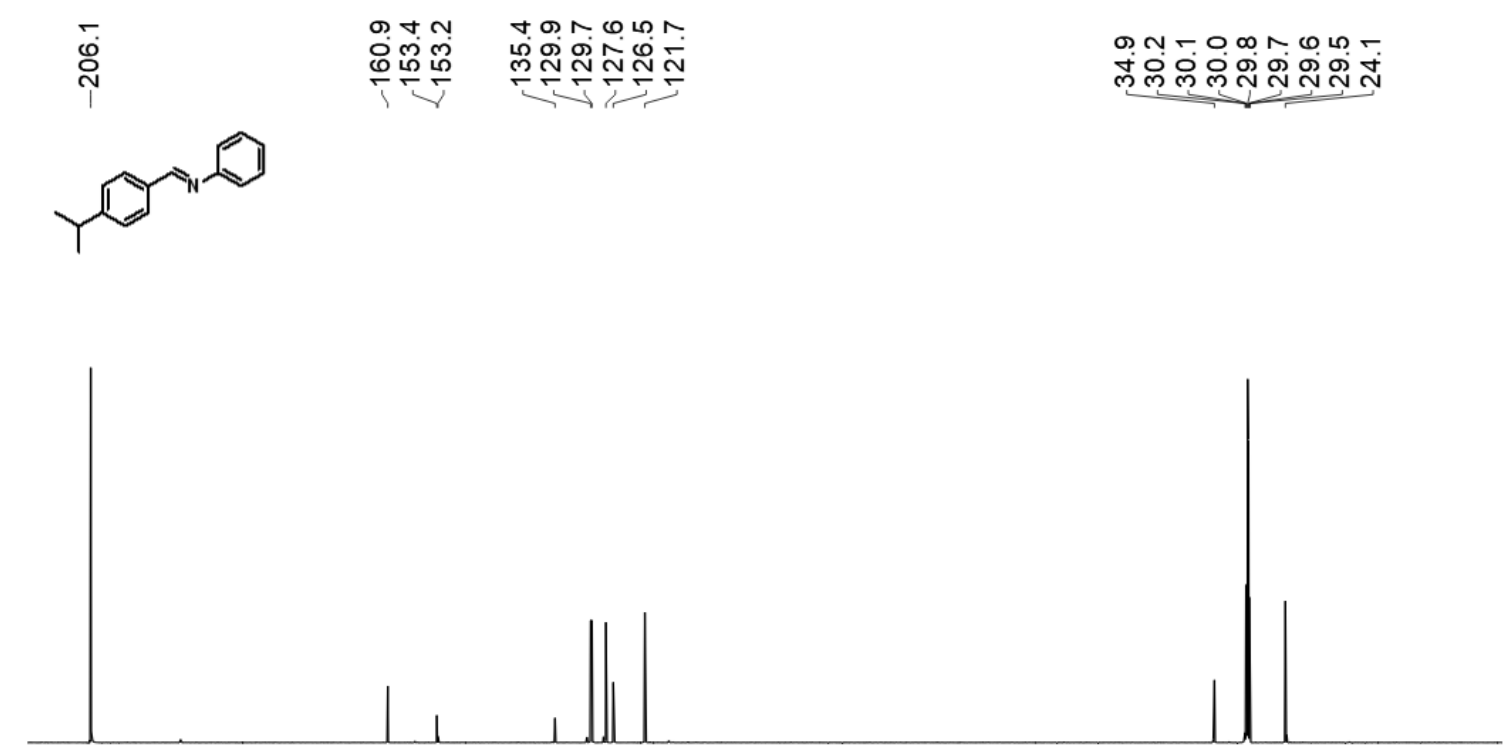

$\begin{array}{llllllllllllllllllllll}210 & 200 & 190 & 180 & 170 & 160 & 150 & 140 & 130 & 120 & \begin{array}{c}110 \\ \mathrm{f} 1(\mathrm{ppm})\end{array} & 90 & 80 & 70 & 60 & 50 & 40 & 30 & 20 & 10 & 0\end{array}$

Figure S27. ${ }^{13} \mathrm{C}$ NMR spectrum of $\mathbf{5 i}\left(\mathrm{CD}_{3} \mathrm{COCD}_{3}, 150 \mathrm{MHz}, 23{ }^{\circ} \mathrm{C}\right)$.
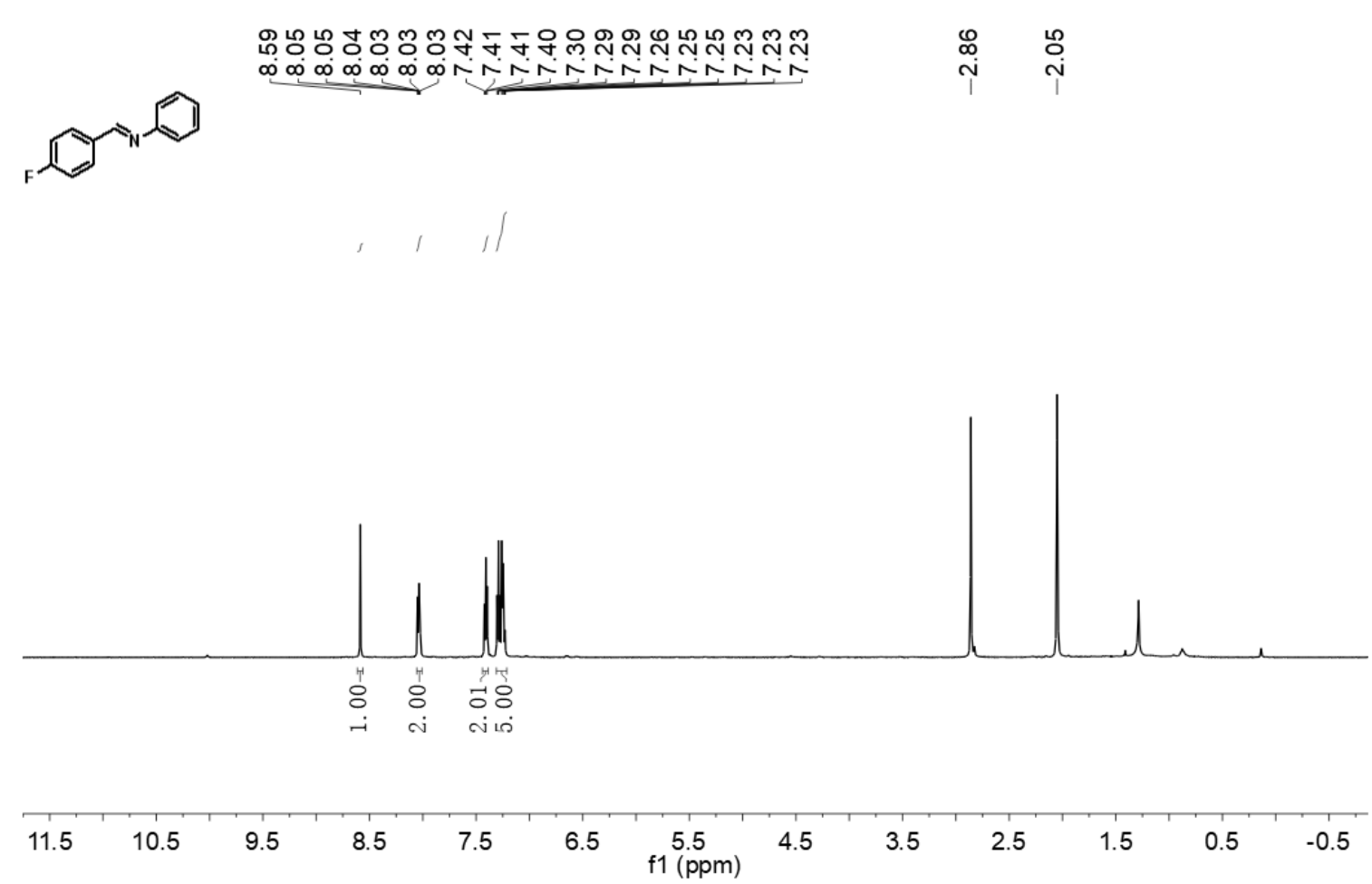

Figure S28. ${ }^{1} \mathrm{H}$ NMR spectrum of $\mathbf{5 j}\left(\mathrm{CD}_{3} \mathrm{COCD}_{3}, 600 \mathrm{MHz}, 23{ }^{\circ} \mathrm{C}\right)$. 

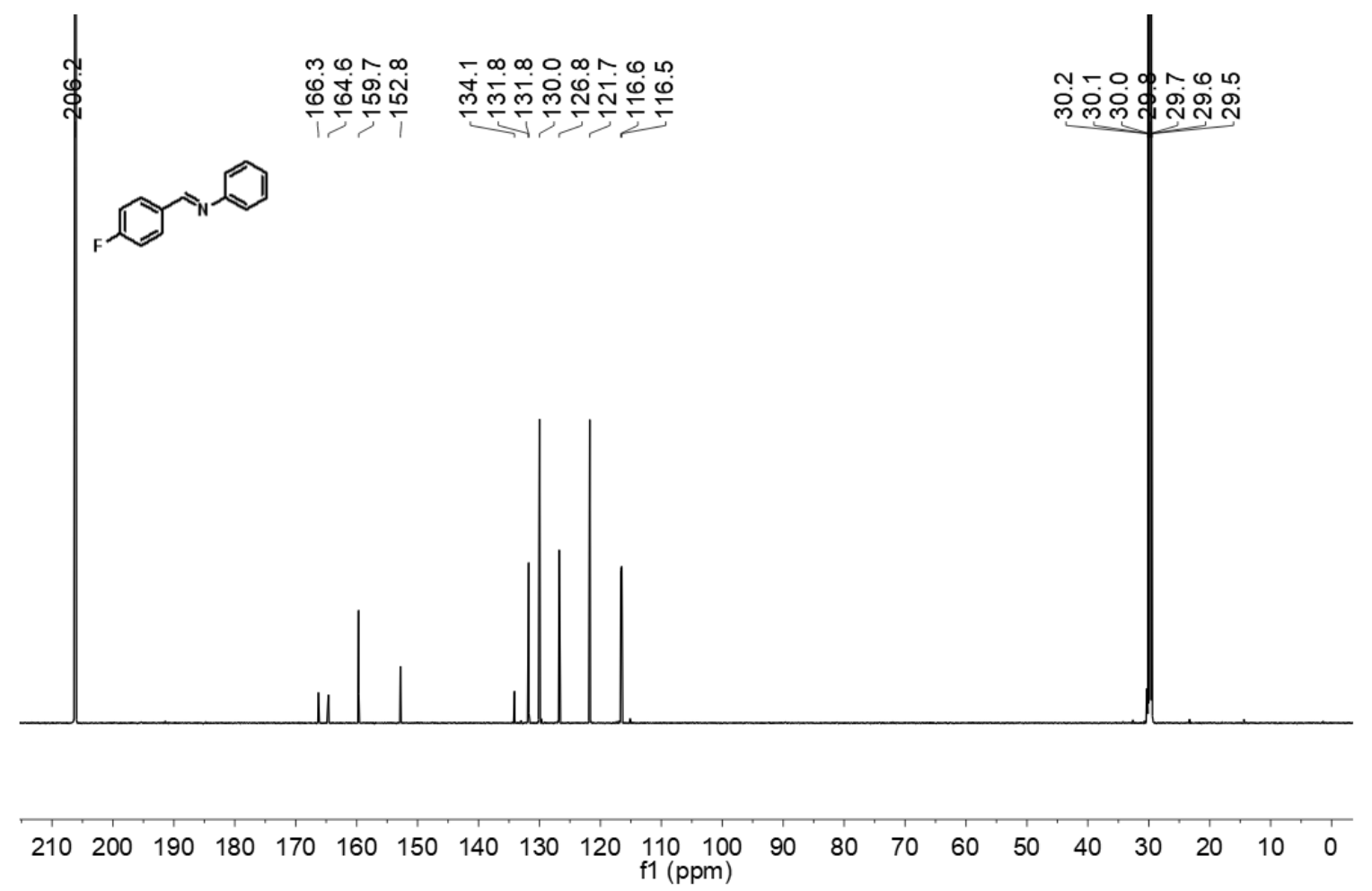

Figure S29. ${ }^{13} \mathrm{C}$ NMR spectrum of $\mathbf{5 j}\left(\mathrm{CD}_{3} \mathrm{COCD}_{3}, 150 \mathrm{MHz}, 23{ }^{\circ} \mathrm{C}\right)$.

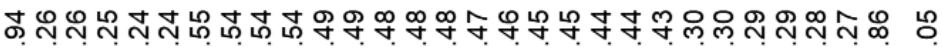

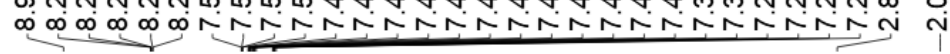
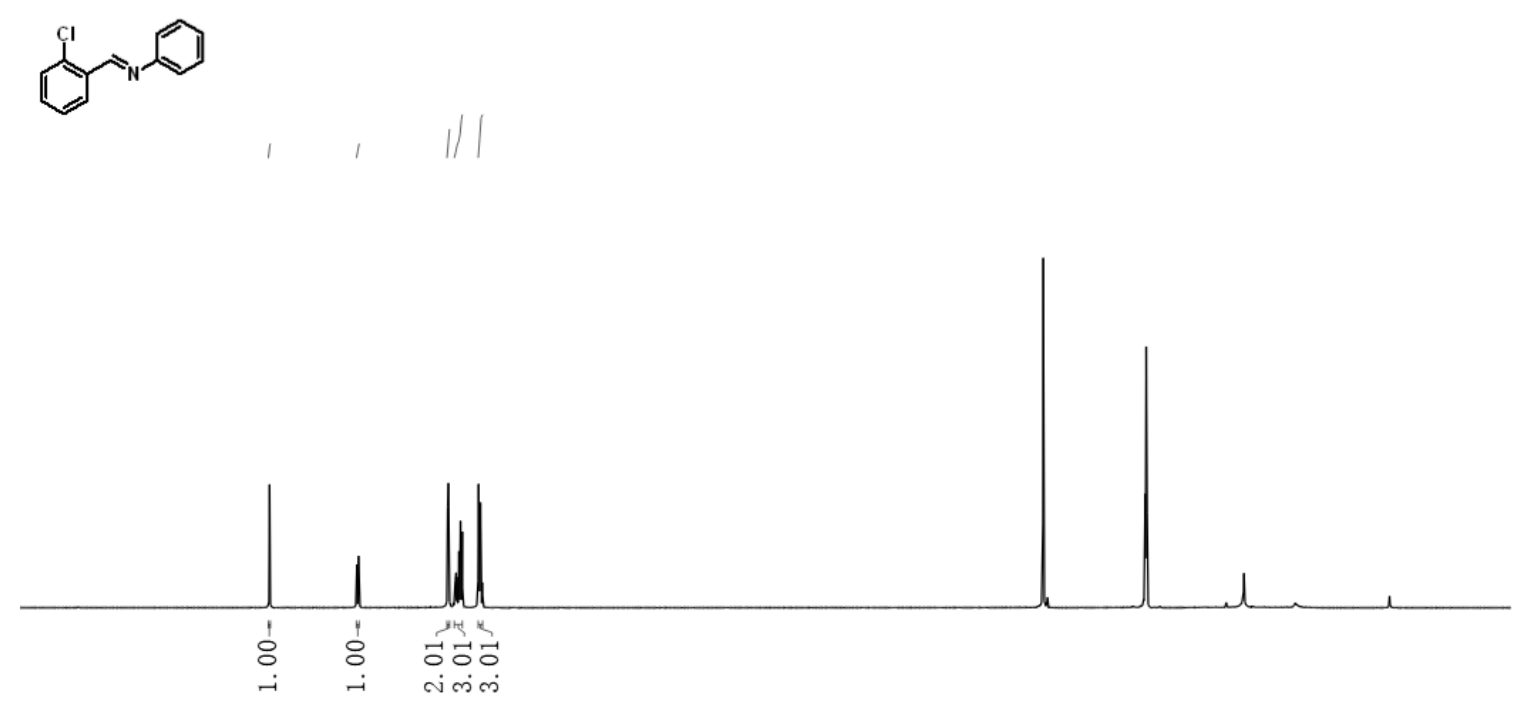

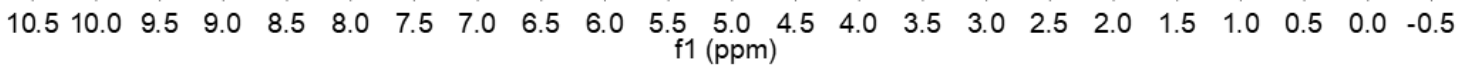

Figure S30. ${ }^{1} \mathrm{H}$ NMR spectrum of $\mathbf{5 k}\left(\mathrm{CD}_{3} \mathrm{COCD}_{3}, 600 \mathrm{MHz}, 23{ }^{\circ} \mathrm{C}\right)$. 


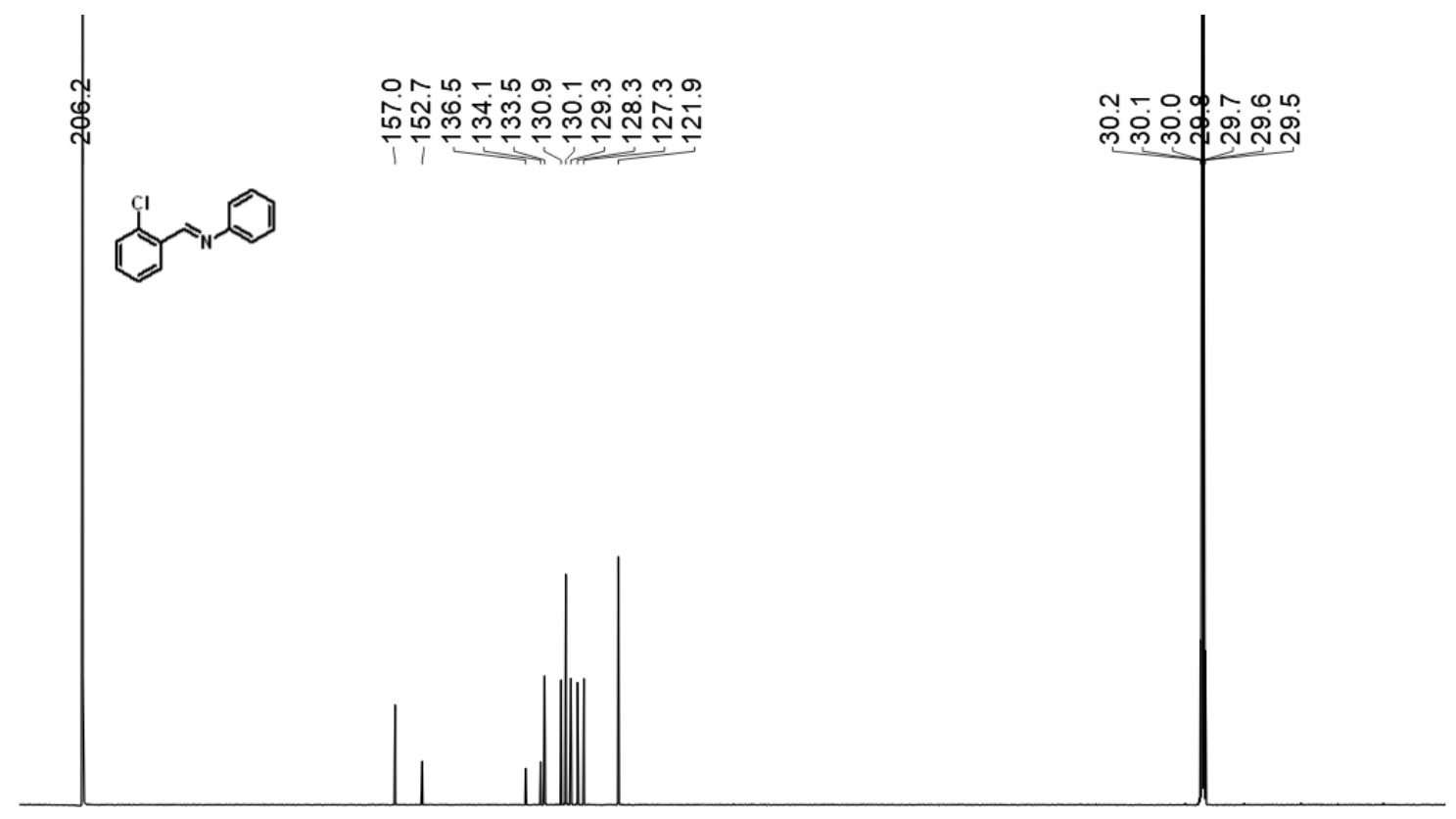

$\begin{array}{lllllllllllllllllllllll}210 & 200 & 190 & 180 & 170 & 160 & 150 & 140 & 130 & 120 & \begin{array}{r}110 \\ \mathrm{f} 1(\mathrm{ppm})\end{array} & 100 & 90 & 80 & 70 & 60 & 50 & 40 & 30 & 20 & 10 & 0\end{array}$

Figure S31. ${ }^{13} \mathrm{C}$ NMR spectrum of $\mathbf{5 k}\left(\mathrm{CD}_{3} \mathrm{COCD}_{3}, 150 \mathrm{MHz}, 23{ }^{\circ} \mathrm{C}\right)$.

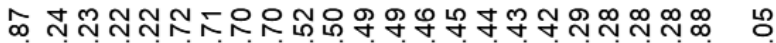

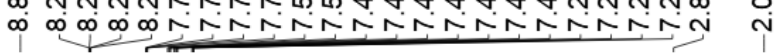
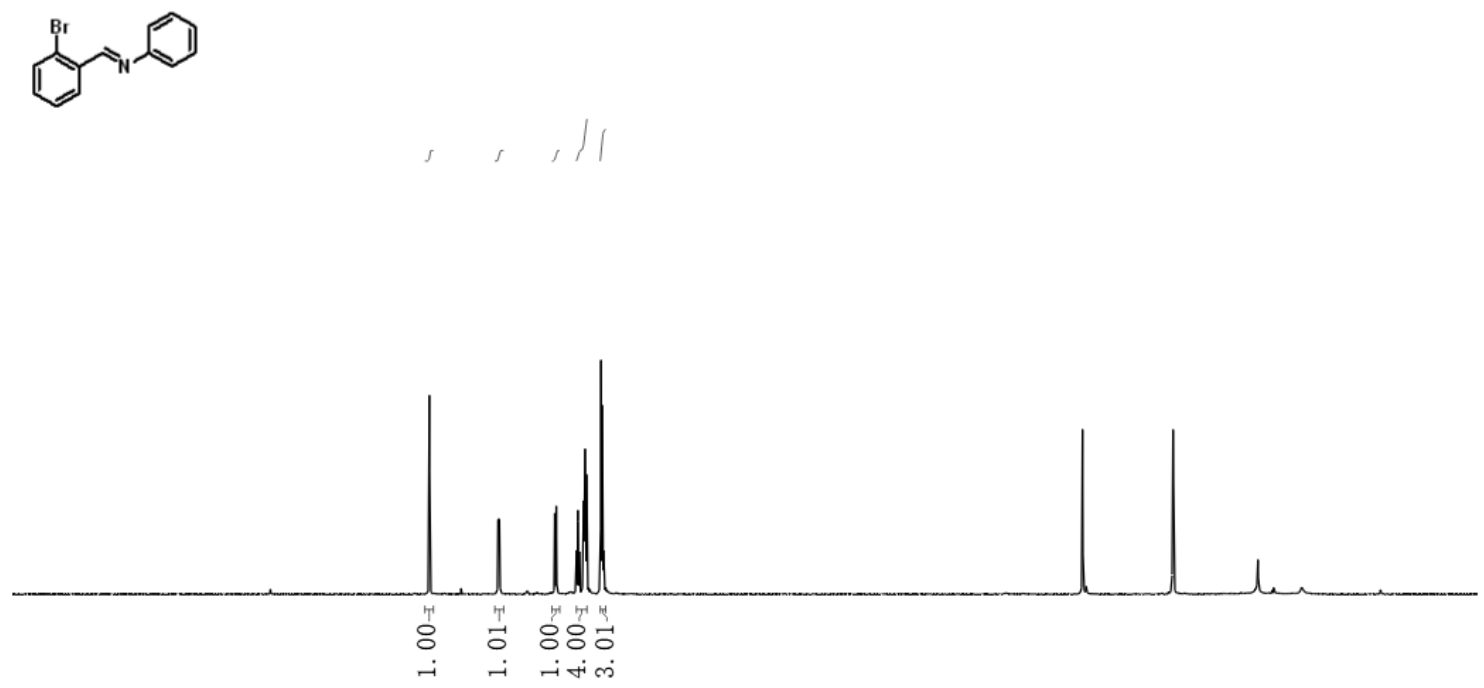

$\begin{array}{llllllllllllll}12.5 & 11.5 & 10.5 & 9.5 & 8.5 & 7.5 & 6.5 & 6.5 & 4.5 & 3.5 & 2.5 & 1.5 & 0.5 & -0.5\end{array}$

Figure S32. ${ }^{1} \mathrm{H}$ NMR spectrum of $\mathbf{5 l}\left(\mathrm{CD}_{3} \mathrm{COCD}_{3}, 600 \mathrm{MHz}, 23{ }^{\circ} \mathrm{C}\right)$. 
:<smiles>Brc1ccccc1C=Nc1ccccc1</smiles>

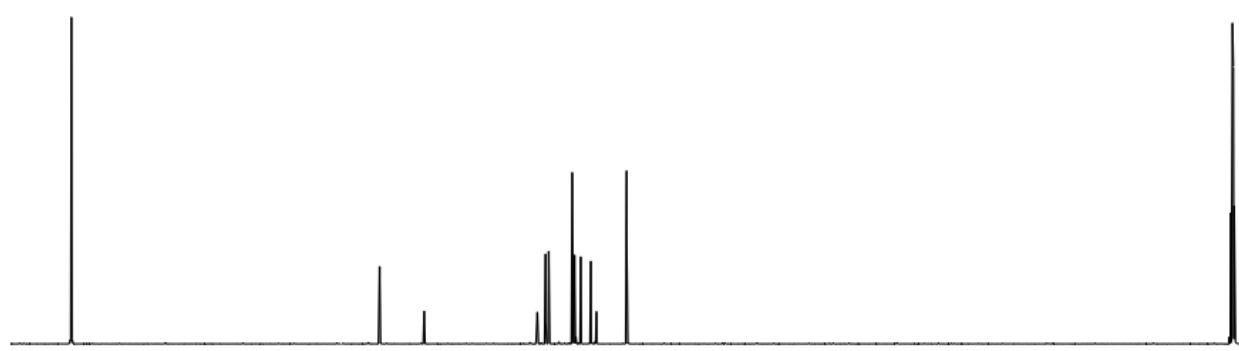

$\begin{array}{llllllllllllllllllllll}210 & 200 & 190 & 180 & 170 & 160 & 150 & 140 & 130 & 120 & \begin{array}{c}110 \\ \mathrm{f} 1(\mathrm{ppm})\end{array} & 100 & 80 & 70 & 60 & 50 & 40 & 30 & 20 & 10 & 0\end{array}$

Figure S33. ${ }^{13} \mathrm{C}$ NMR spectrum of $\mathbf{5 l}\left(\mathrm{CD}_{3} \mathrm{COCD}_{3}, 150 \mathrm{MHz}, 23{ }^{\circ} \mathrm{C}\right)$.

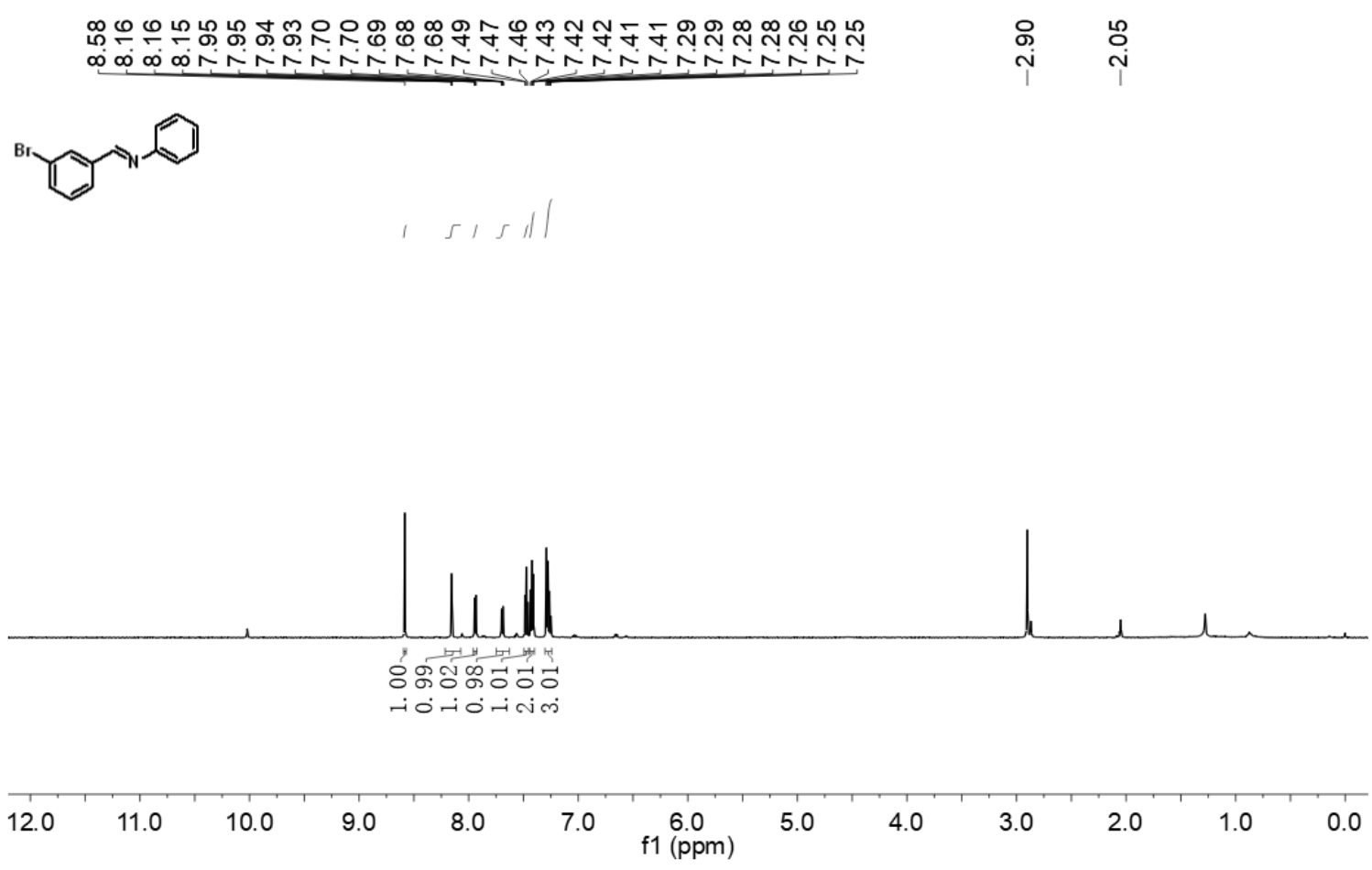

Figure S34. ${ }^{1} \mathrm{H}$ NMR spectrum of $\mathbf{5 m}\left(\mathrm{CD}_{3} \mathrm{COCD}_{3}, 600 \mathrm{MHz}, 23{ }^{\circ} \mathrm{C}\right)$. 

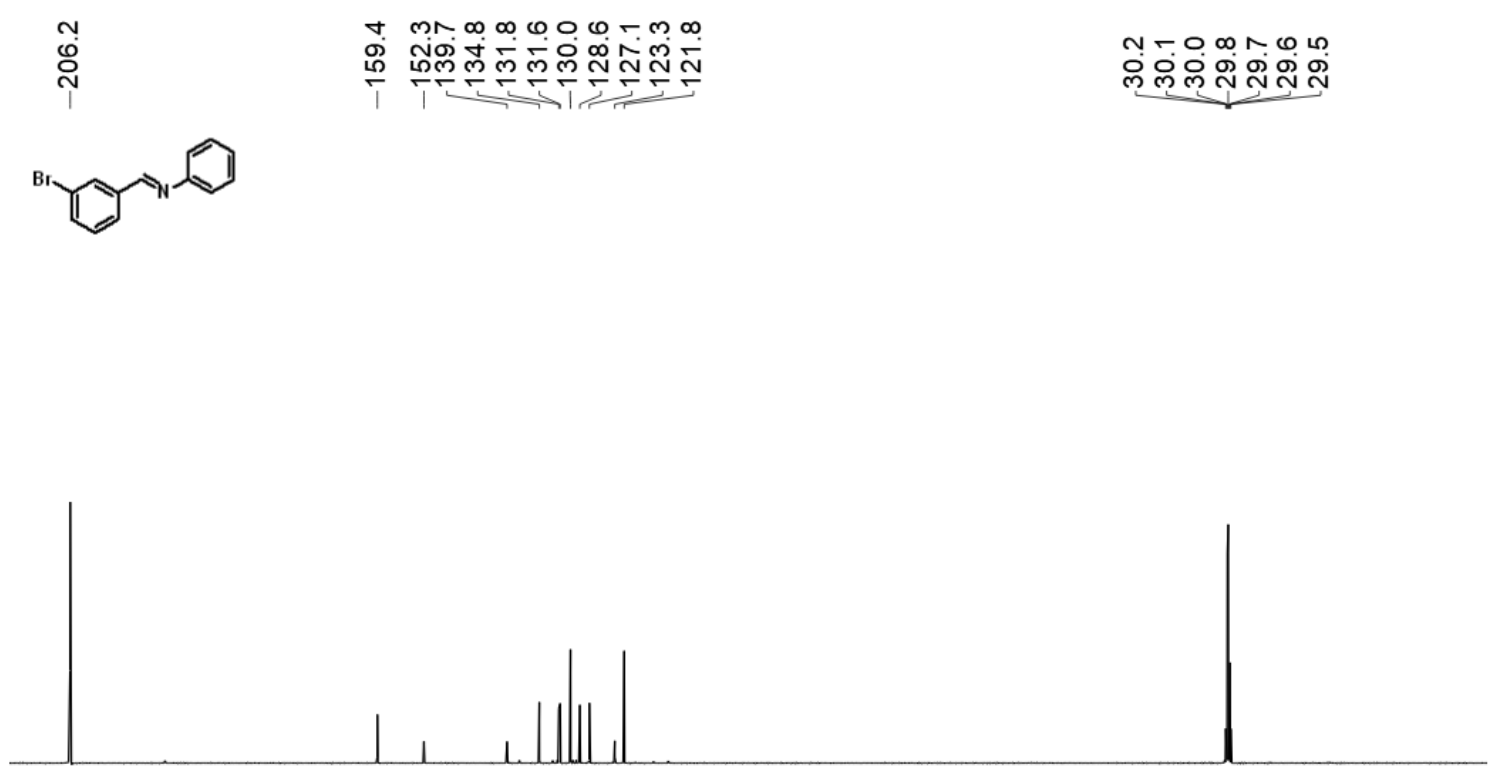

$\begin{array}{llllllllllllllllllllll}210 & 200 & 190 & 180 & 170 & 160 & 150 & 140 & 130 & 120 & \begin{array}{c}110 \quad 100 \\ \mathrm{f} 1(\mathrm{ppm})\end{array} & 90 & 80 & 70 & 60 & 50 & 40 & 30 & 20 & 10 & 0\end{array}$

Figure S35. ${ }^{13} \mathrm{C}$ NMR spectrum of $\mathbf{5 m}\left(\mathrm{CD}_{3} \mathrm{COCD}_{3}, 150 \mathrm{MHz}, 23{ }^{\circ} \mathrm{C}\right)$.

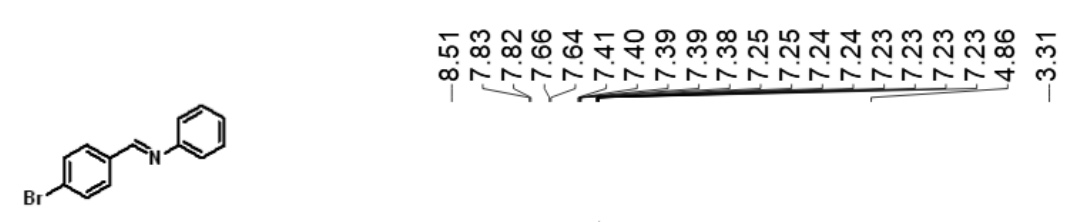

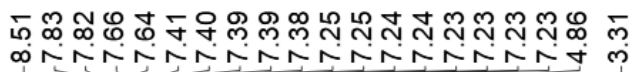

满

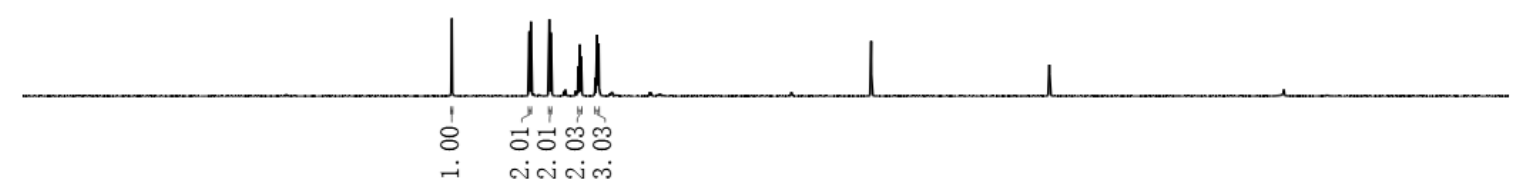

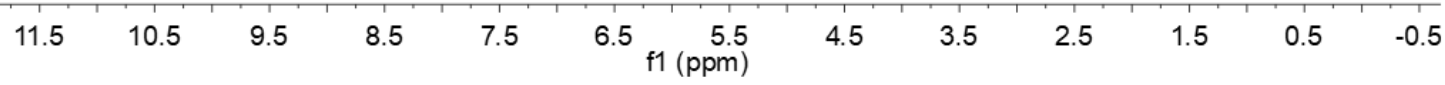

Figure S36. ${ }^{1} \mathrm{H}$ NMR spectrum of $\mathbf{5 n}\left(\mathrm{CD}_{3} \mathrm{OD}, 600 \mathrm{MHz}, 23{ }^{\circ} \mathrm{C}\right)$. 


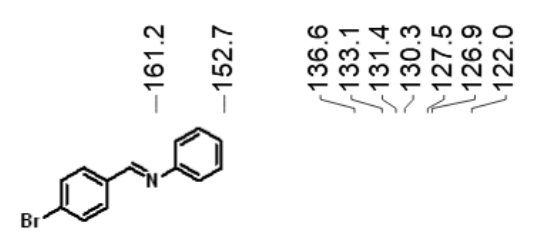

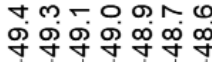

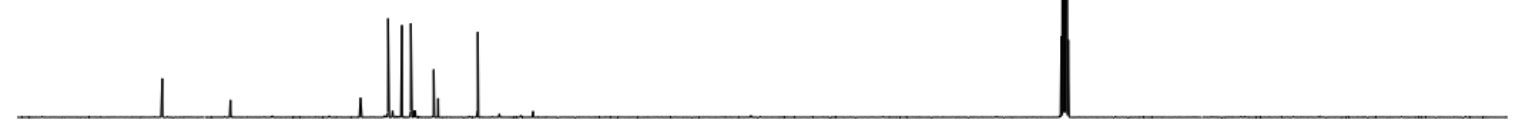

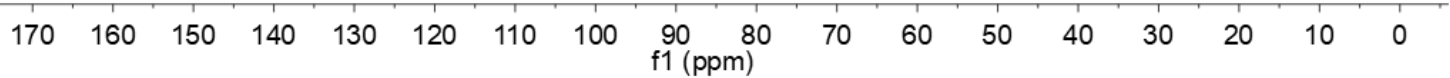

Figure S37. ${ }^{13} \mathrm{C}$ NMR spectrum of $\mathbf{5 n}\left(\mathrm{CD}_{3} \mathrm{OD}, 150 \mathrm{MHz}, 23{ }^{\circ} \mathrm{C}\right)$.

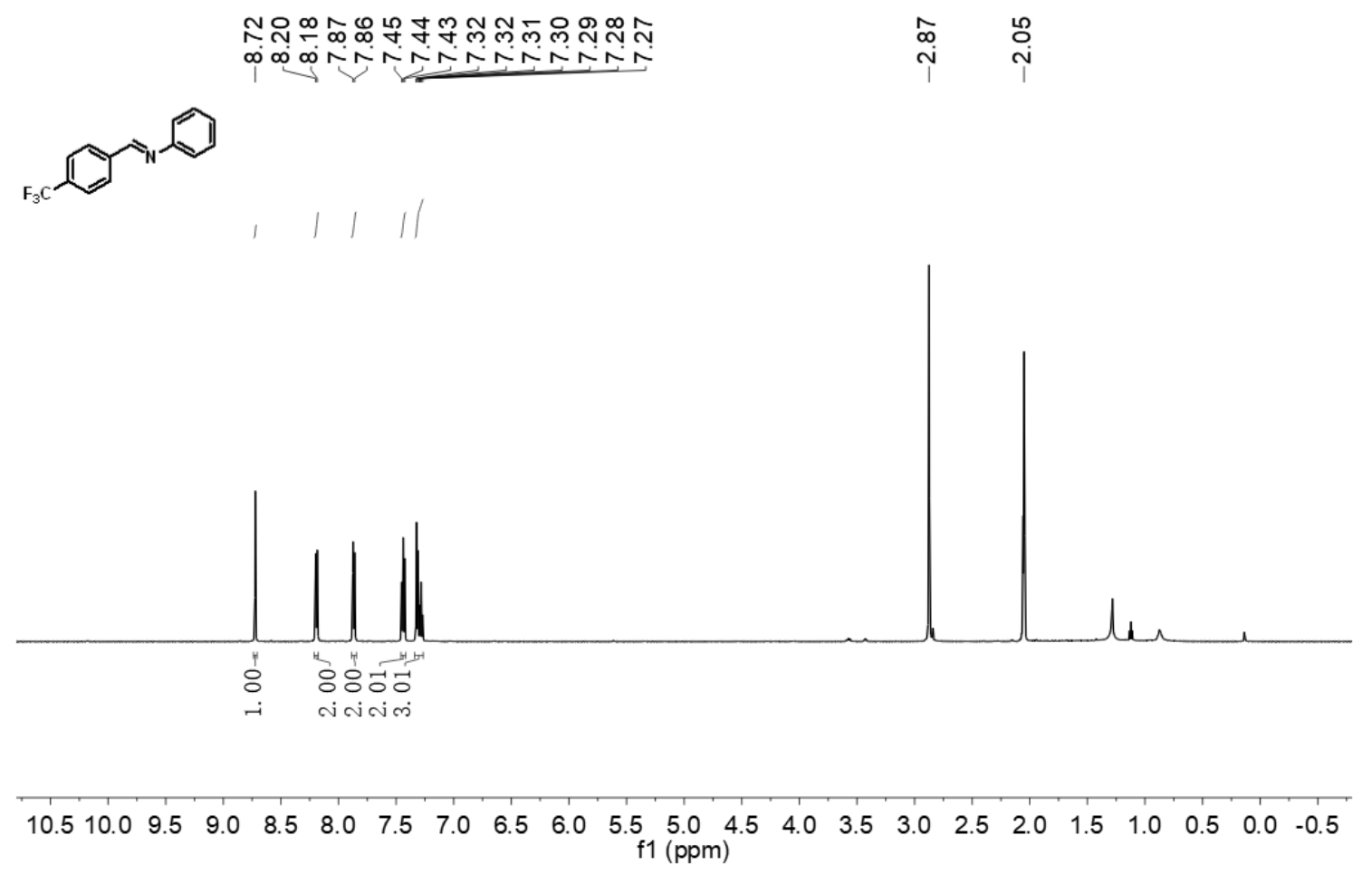

Figure S38. ${ }^{1} \mathrm{H}$ NMR spectrum of $\mathbf{5 o}\left(\mathrm{CD}_{3} \mathrm{COCD}_{3}, 600 \mathrm{MHz}, 2{ }^{\circ} \mathrm{C}\right)$. 


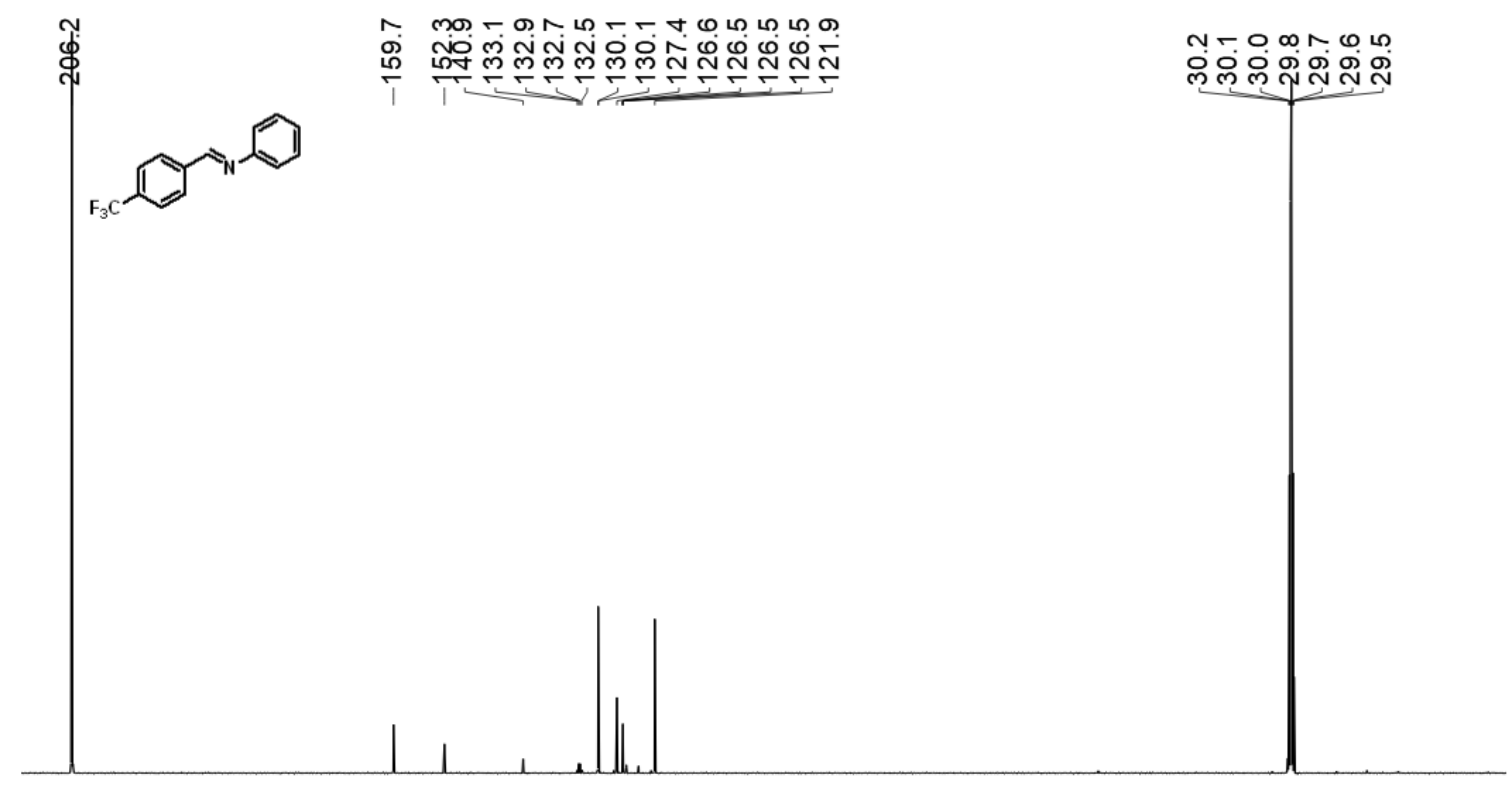

$\begin{array}{llllllllllllllllllllll}210 & 200 & 190 & 180 & 170 & 160 & 150 & 140 & 130 & 120 & \begin{array}{c}110 \\ \mathrm{f} 1(\mathrm{ppm})\end{array} & 90 & 80 & 70 & 60 & 50 & 40 & 30 & 20 & 10 & 0\end{array}$

Figure S39. ${ }^{13} \mathrm{C}$ NMR spectrum of $\mathbf{5 o}\left(\mathrm{CD}_{3} \mathrm{COCD}_{3}, 150 \mathrm{MHz}, 23{ }^{\circ} \mathrm{C}\right)$.

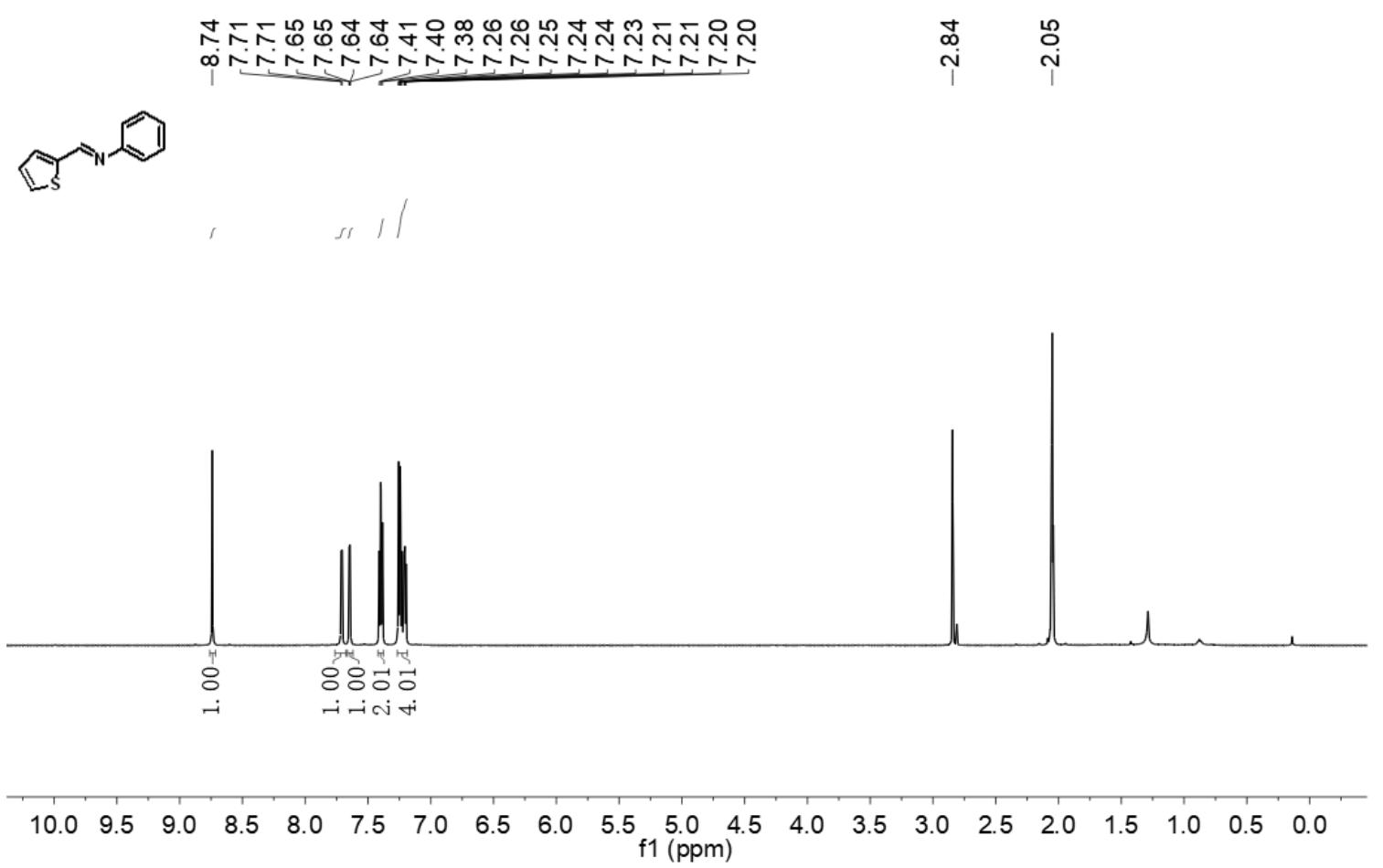

Figure S40. ${ }^{1} \mathrm{H}$ NMR spectrum of $\mathbf{5 p}\left(\mathrm{CD}_{3} \mathrm{COCD}_{3}, 600 \mathrm{MHz}, 23{ }^{\circ} \mathrm{C}\right)$. 


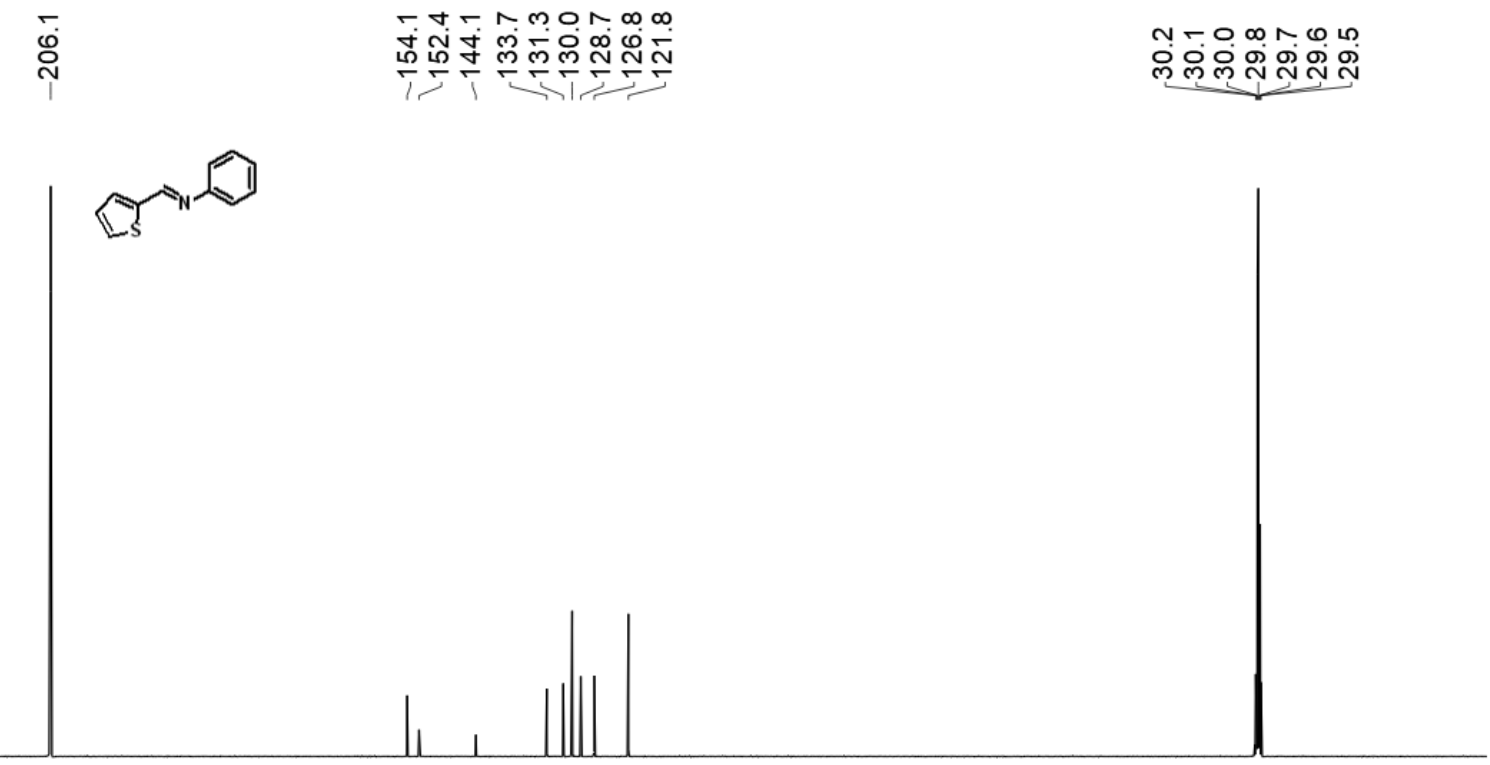

$\begin{array}{lllllllllllllllllllll}210 & 200 & 190 & 180 & 170 & 160 & 150 & 140 & 130 & 120 & \begin{array}{l}110 \\ \mathrm{f} 1(\mathrm{ppm})\end{array} & 90 & 80 & 70 & 60 & 50 & 40 & 30 & 20 & 10 & 0\end{array}$

Figure S41. ${ }^{1} \mathrm{H}$ NMR spectrum of $\mathbf{5 p}\left(\mathrm{CD}_{3} \mathrm{COCD}_{3}, 150 \mathrm{MHz}, 23{ }^{\circ} \mathrm{C}\right)$.

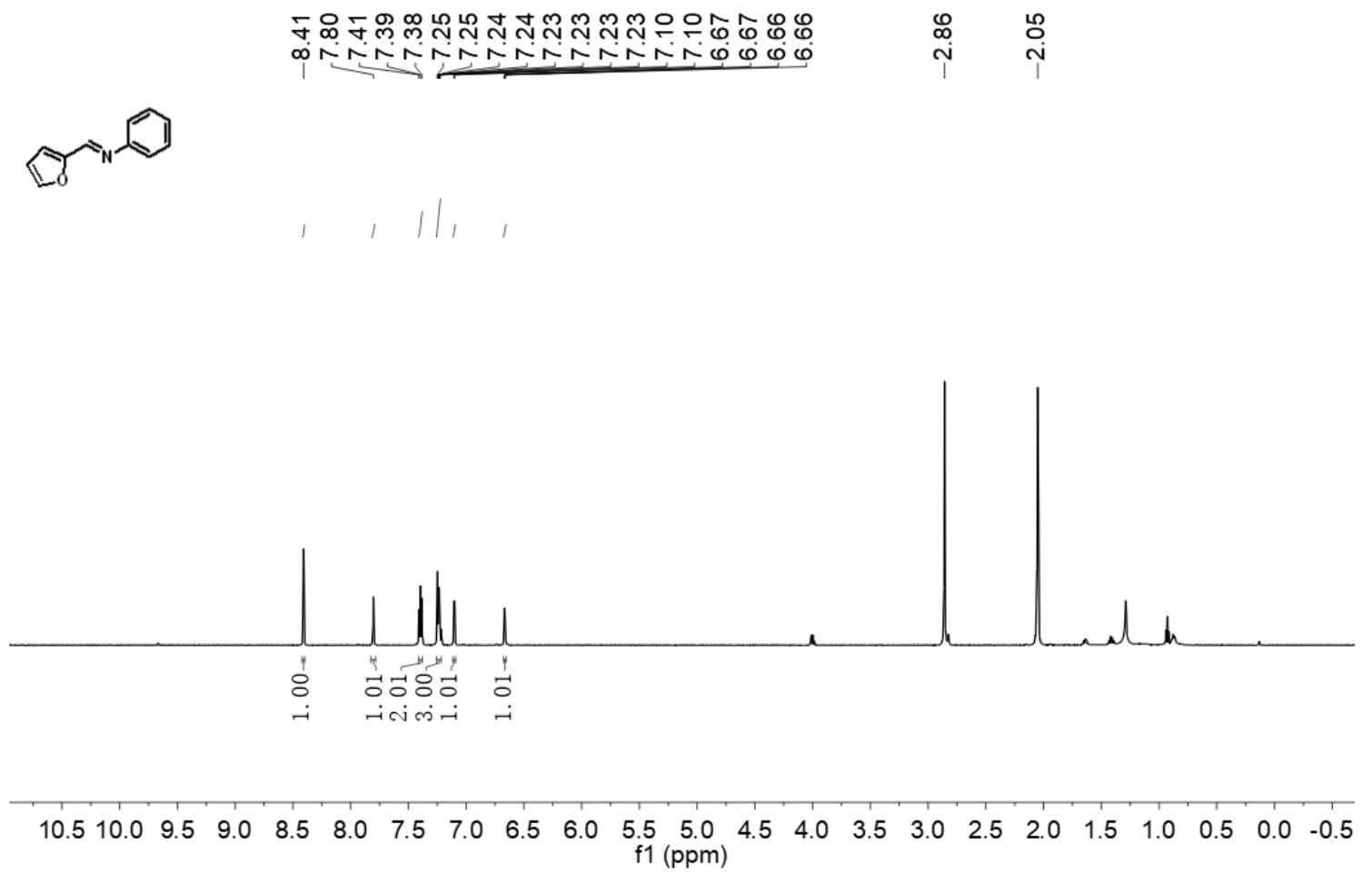

Figure S42. ${ }^{1} \mathrm{H}$ NMR spectrum of $\mathbf{5 q}\left(\mathrm{CD}_{3} \mathrm{COCD}_{3}, 600 \mathrm{MHz}, 23{ }^{\circ} \mathrm{C}\right)$. 


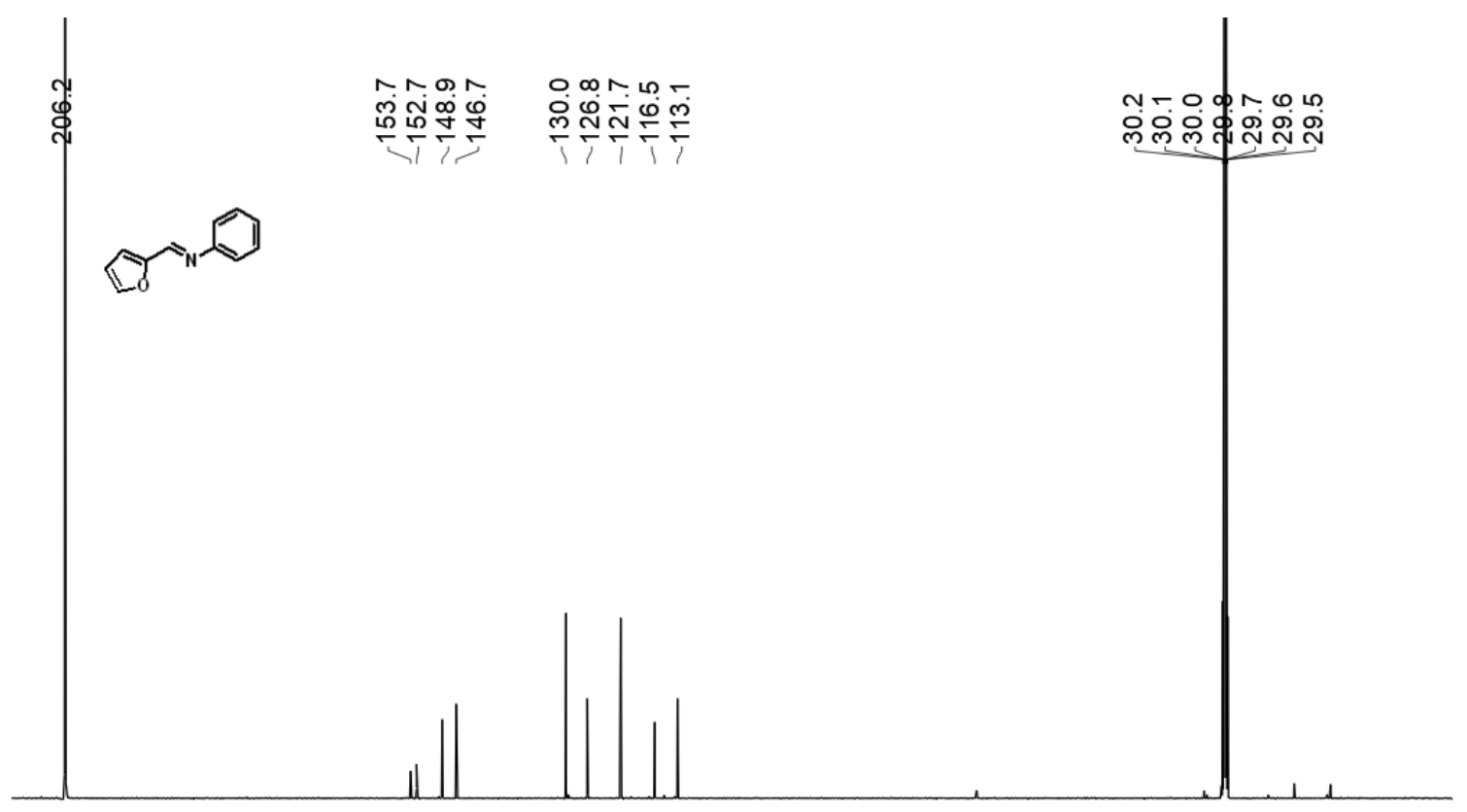

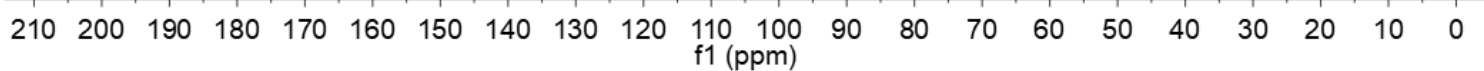

Figure S43. ${ }^{13} \mathrm{C}$ NMR spectrum of $\mathbf{5 q}\left(\mathrm{CD}_{3} \mathrm{COCD}_{3}, 150 \mathrm{MHz}, 23{ }^{\circ} \mathrm{C}\right)$.

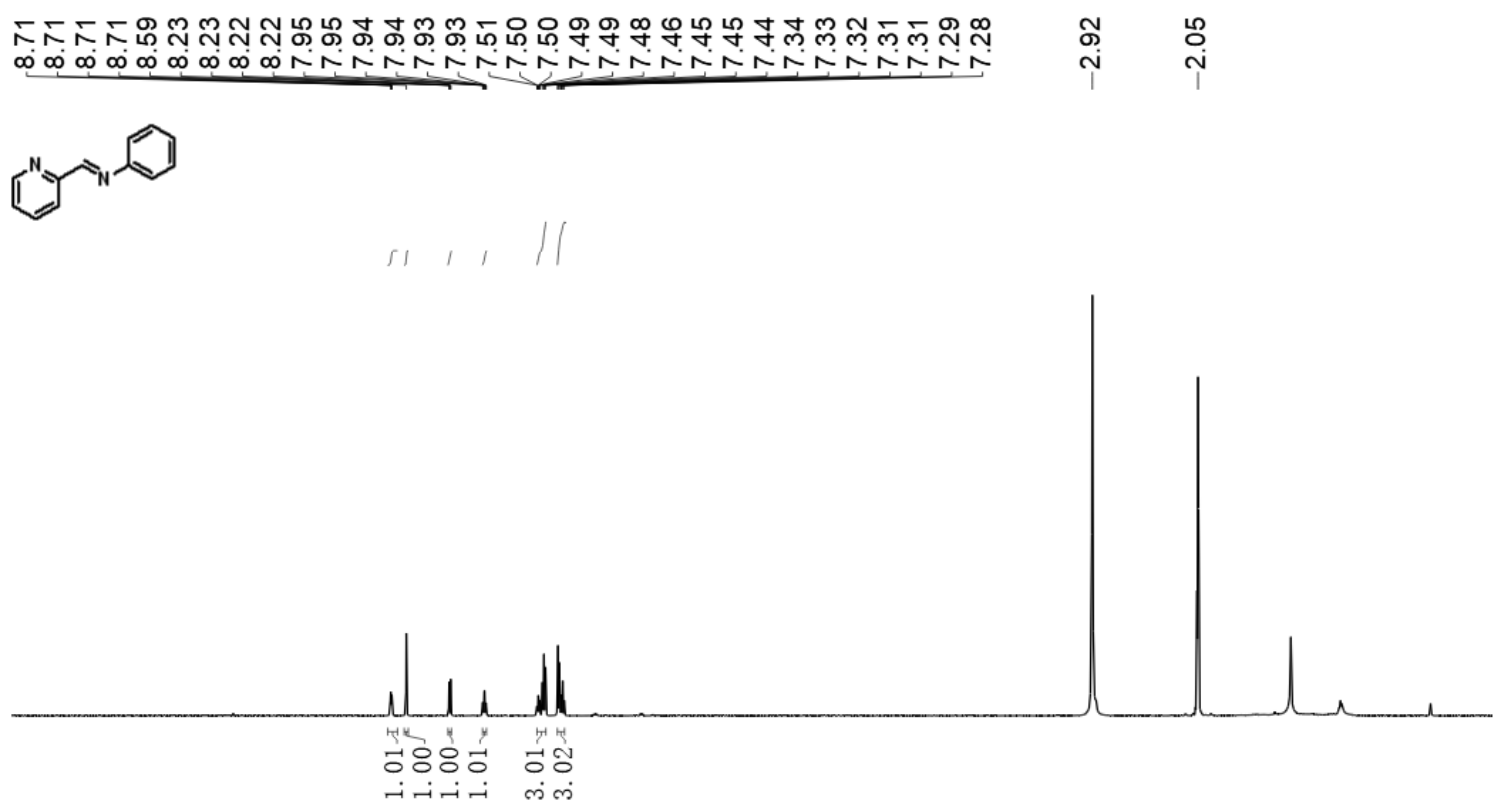

$\begin{array}{lllllllllllllllllllllllll}11.5 & 11.0 & 10.5 & 10.0 & 9.5 & 9.0 & 8.5 & 8.0 & 7.5 & 7.0 & 6.5 & 6.0 & 5.5 & 5.0 & 4.5 & 4.0 & 3.5 & 3.0 & 2.5 & 2.0 & 1.5 & 1.0 & 0.5 & 0.0\end{array}$

Figure S44. ${ }^{1} \mathrm{H}$ NMR spectrum of $\mathbf{5 r}\left(\mathrm{CD}_{3} \mathrm{COCD}_{3}, 600 \mathrm{MHz}, 23{ }^{\circ} \mathrm{C}\right)$. 

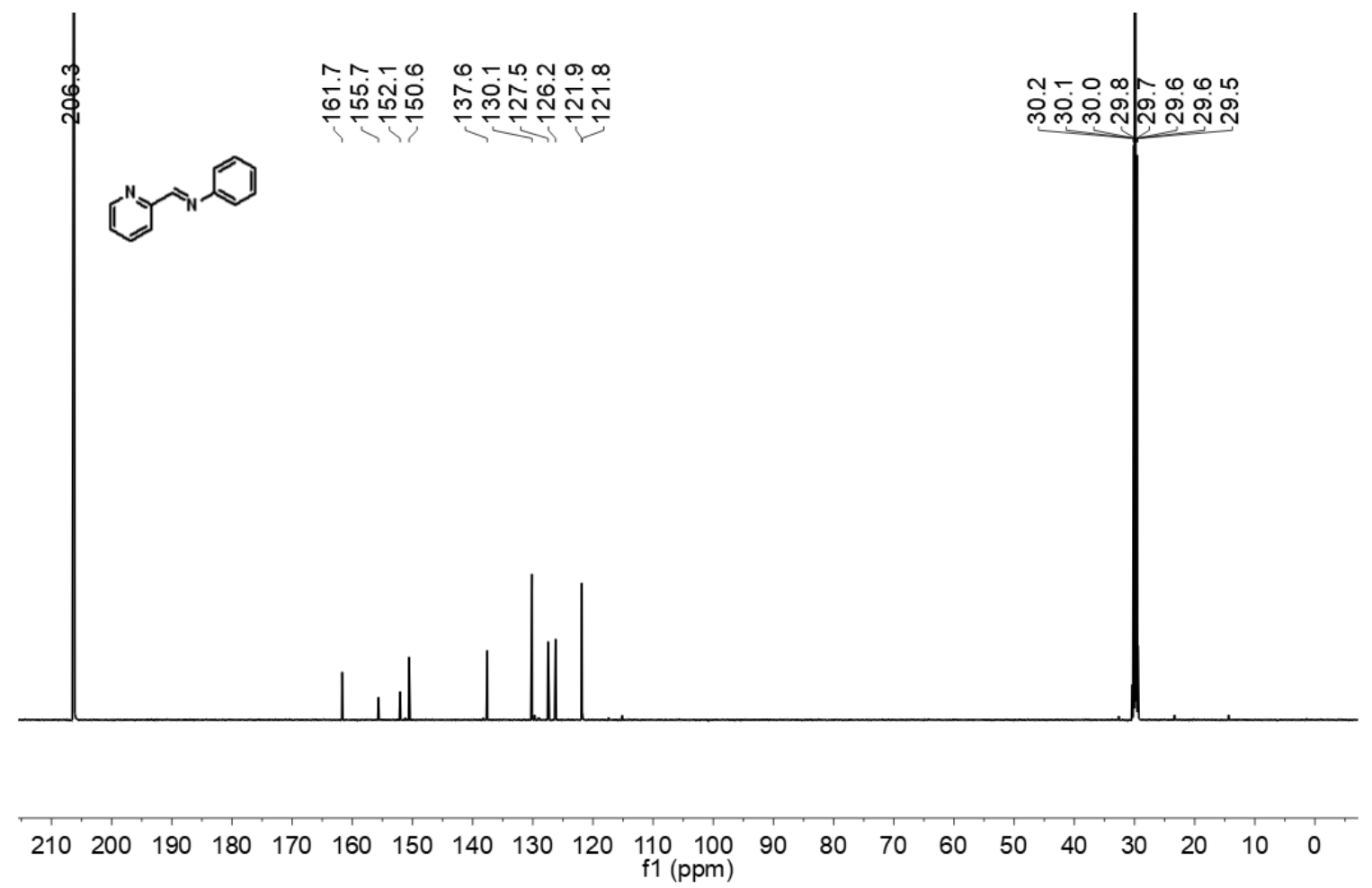

Figure $\mathbf{S 4 5} .{ }^{13} \mathrm{C}$ NMR spectrum of $\mathbf{5 r}\left(\mathrm{CD}_{3} \mathrm{COCD}_{3}, 150 \mathrm{MHz}, 23{ }^{\circ} \mathrm{C}\right)$.
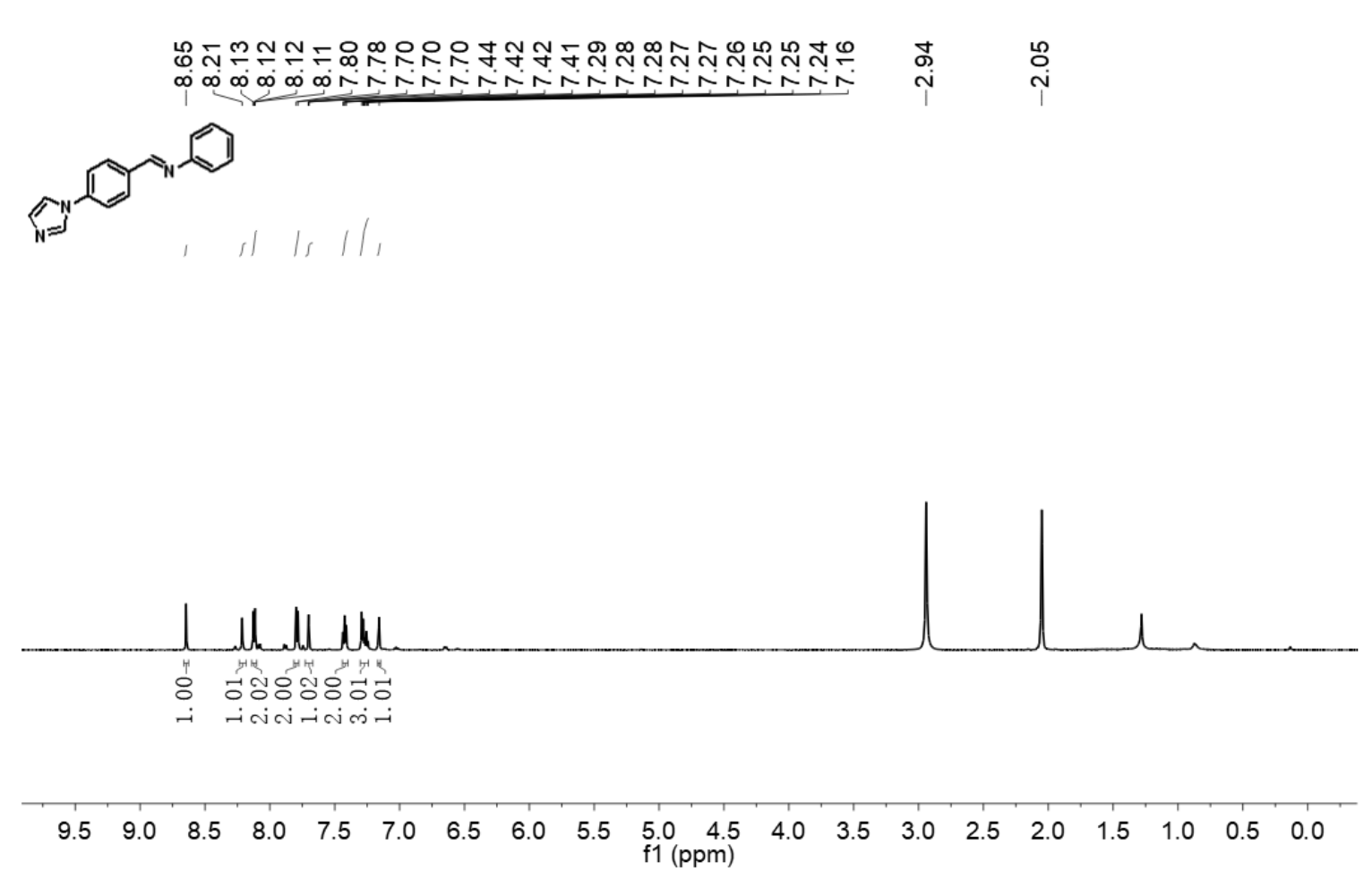

Figure S46. ${ }^{1} \mathrm{H}$ NMR spectrum of $\mathbf{5 s}\left(\mathrm{CD}_{3} \mathrm{COCD}_{3}, 600 \mathrm{MHz}, 23^{\circ} \mathrm{C}\right)$. 


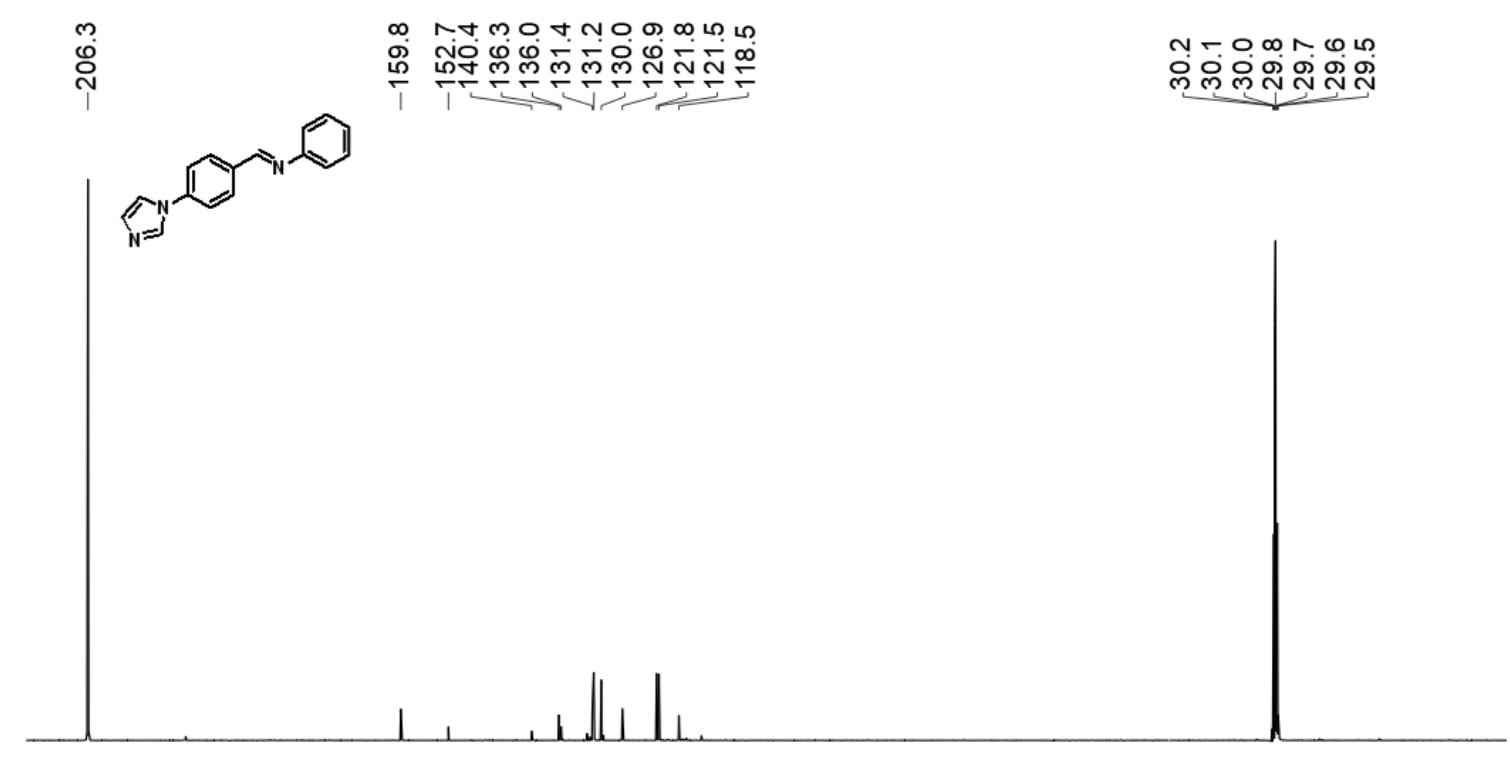

$\begin{array}{llllllllllllllllllllll}210 & 200 & 190 & 180 & 170 & 160 & 150 & 140 & 130 & 120 & \begin{array}{c}110 \\ \mathrm{f} 1(\mathrm{ppm})\end{array} & 90 & 80 & 70 & 60 & 50 & 40 & 30 & 20 & 10 & 0\end{array}$

Figure S47. ${ }^{13} \mathrm{C}$ NMR spectrum of $5 \mathbf{s}\left(\mathrm{CD}_{3} \mathrm{COCD}_{3}, 150 \mathrm{MHz}, 23{ }^{\circ} \mathrm{C}\right)$.

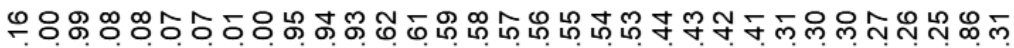

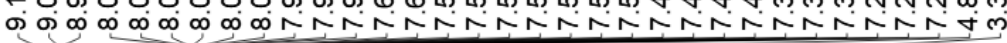
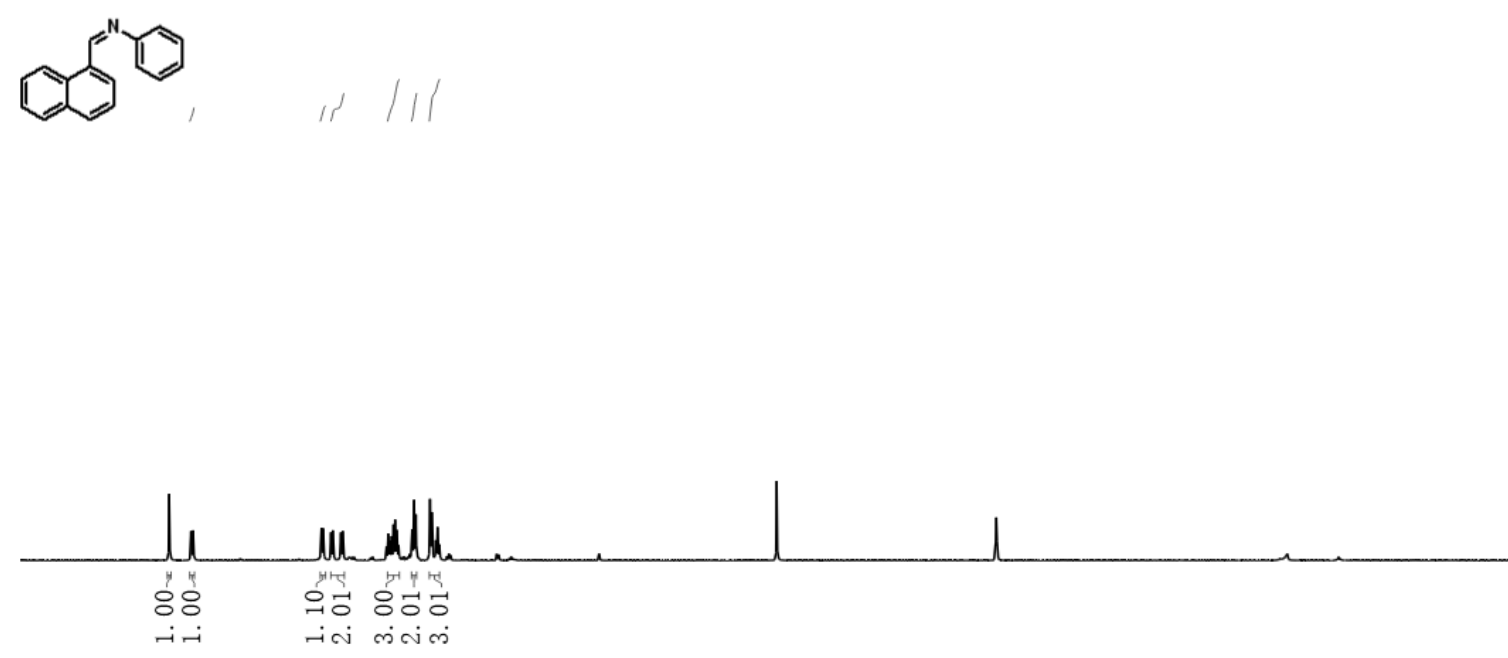

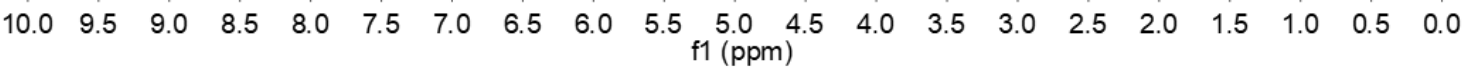

Figure S48. ${ }^{1} \mathrm{H}$ NMR spectrum of $\mathbf{5 t}\left(\mathrm{CD}_{3} \mathrm{OD}, 600 \mathrm{MHz}, 23{ }^{\circ} \mathrm{C}\right)$. 


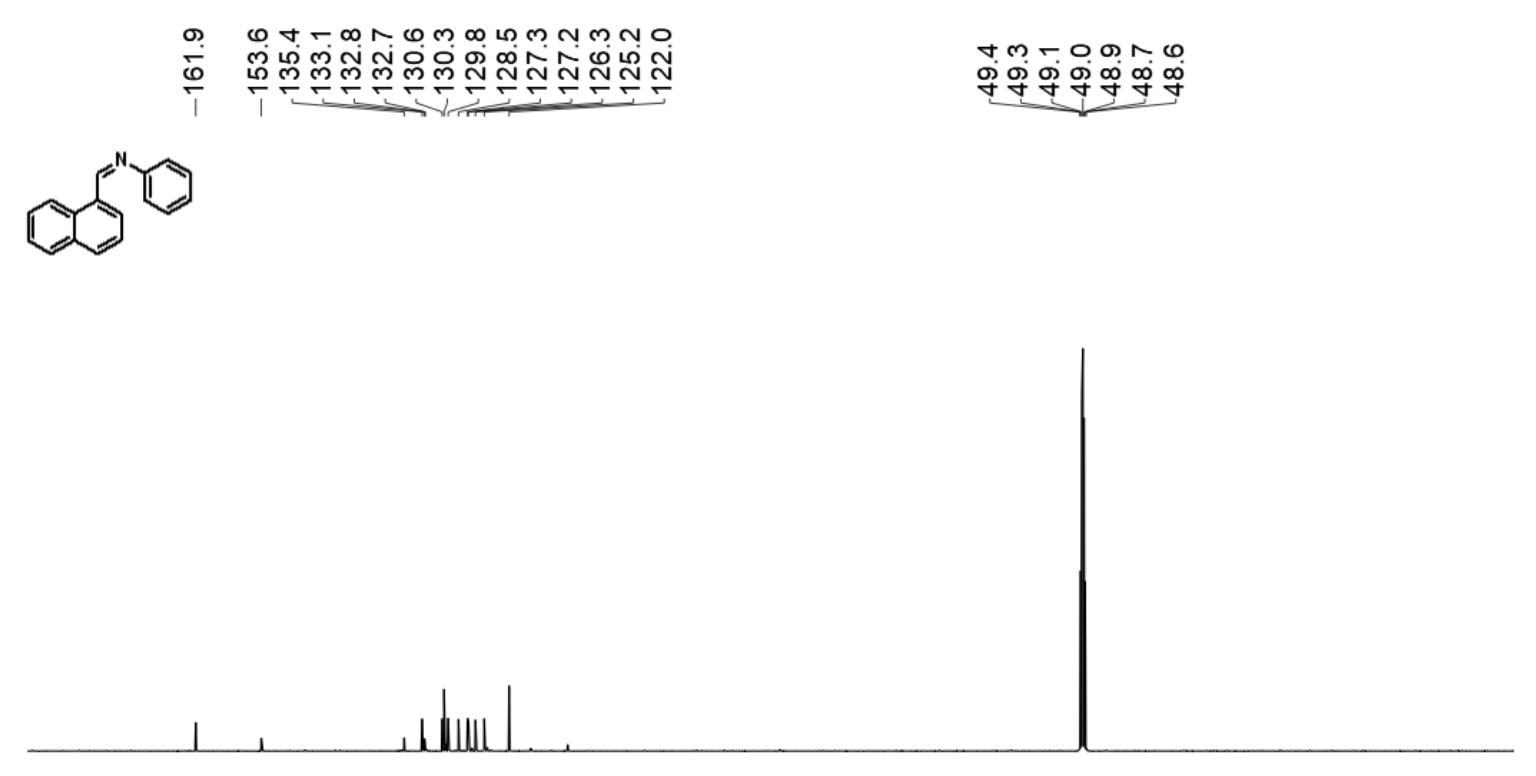

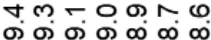

๑.

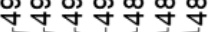

$\begin{array}{lllllllllllllllllll}180 & 170 & 160 & 150 & 140 & 130 & 120 & 110 & 100 & \underset{\mathrm{f} 1(\mathrm{ppm})}{90} & 80 & 70 & 60 & 50 & 40 & 30 & 20 & 10 & 0\end{array}$

Figure S49. ${ }^{13} \mathrm{C}$ NMR spectrum of $\mathbf{5 t}\left(\mathrm{CD}_{3} \mathrm{OD}, 150 \mathrm{MHz}, 23{ }^{\circ} \mathrm{C}\right)$.
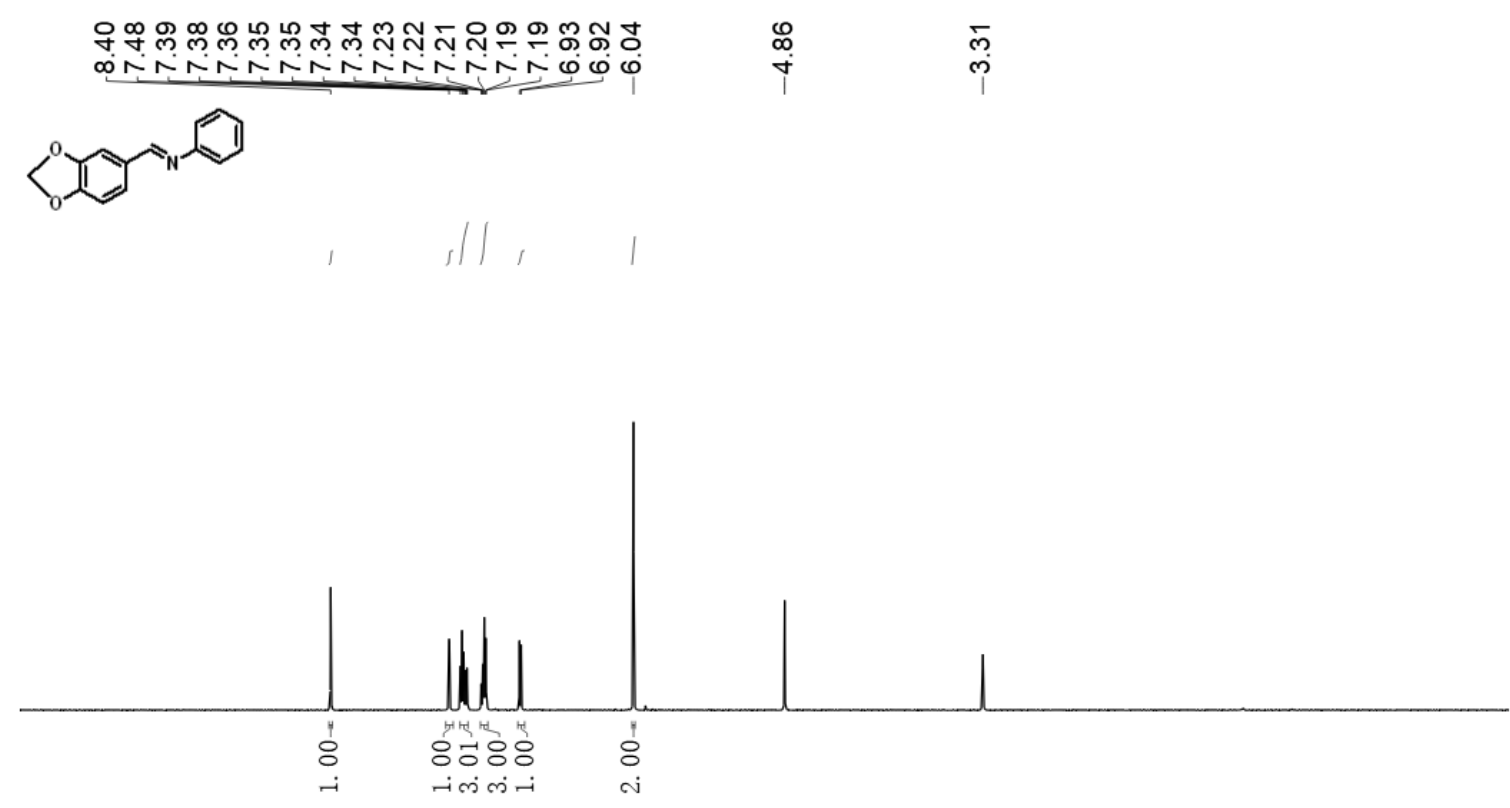

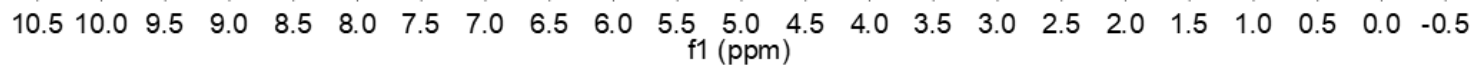

Figure S50. ${ }^{1} \mathrm{H}$ NMR spectrum of $\mathbf{5 u}\left(\mathrm{CD}_{3} \mathrm{OD}, 600 \mathrm{MHz}, 23{ }^{\circ} \mathrm{C}\right)$. 

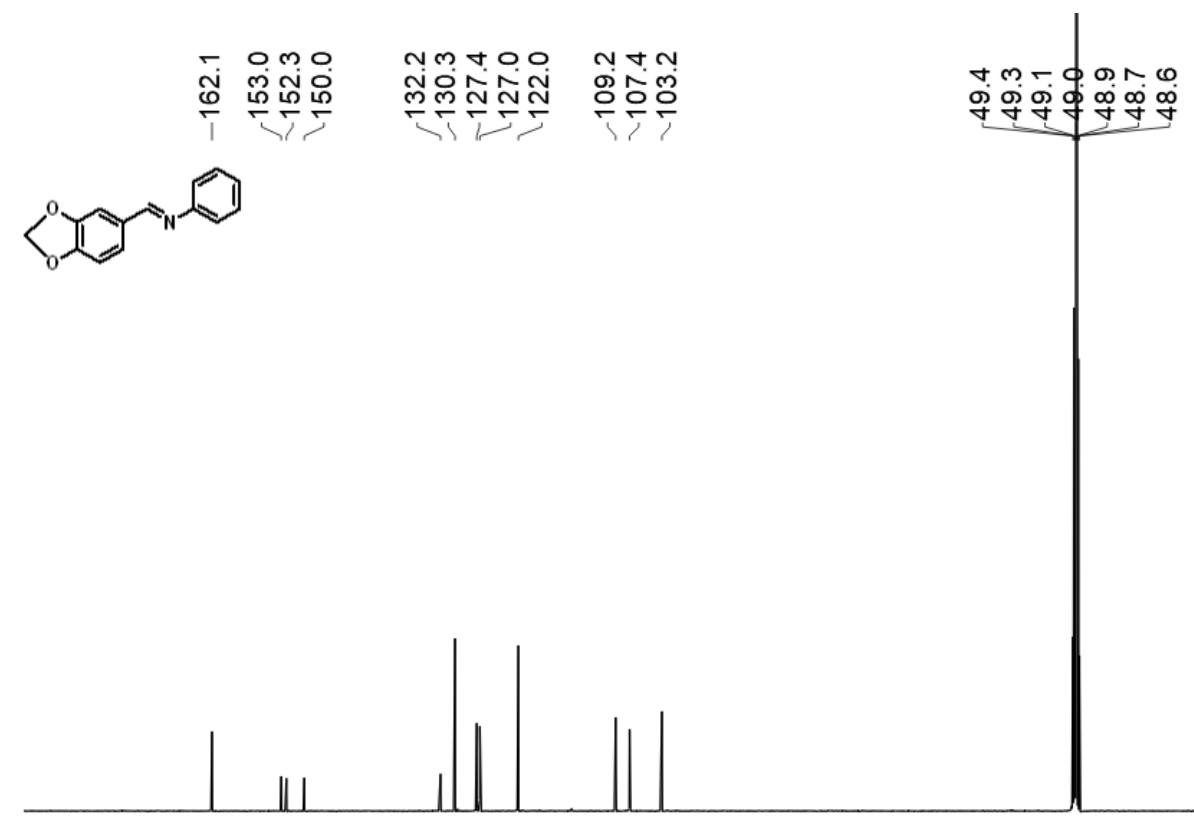

$\begin{array}{llllllllllllllllll}180 & 170 & 160 & 150 & 140 & 130 & 120 & 110 & 100 & \underset{\mathrm{f} 1}{9(\mathrm{ppm})} 80 & 70 & 60 & 50 & 40 & 30 & 20 & 10 & 0\end{array}$

Figure S51. ${ }^{13} \mathrm{C}$ NMR spectrum of $\mathbf{5 u}\left(\mathrm{CD}_{3} \mathrm{OD}, 150 \mathrm{MHz}, 23{ }^{\circ} \mathrm{C}\right)$.

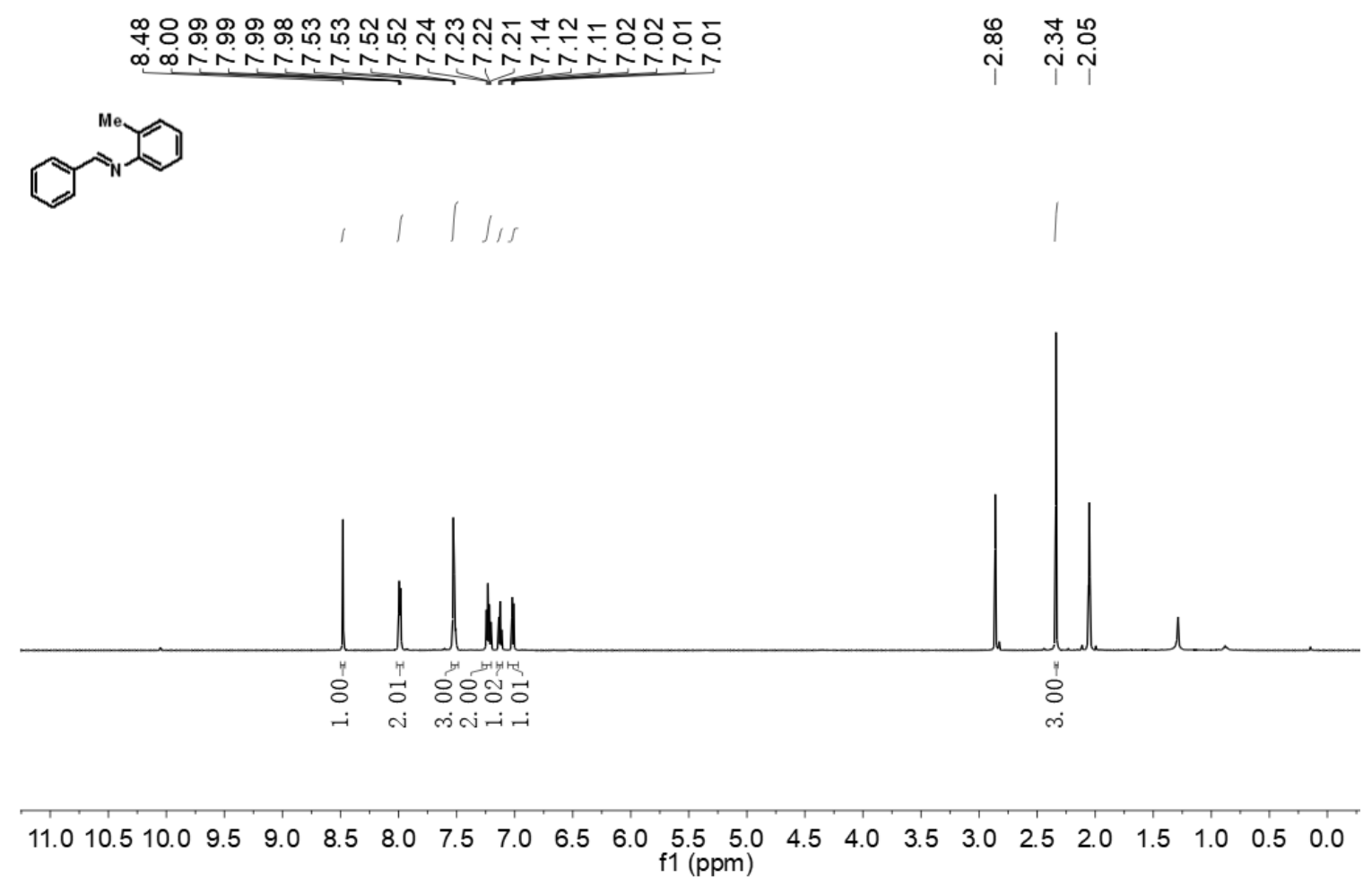

Figure S52. ${ }^{1} \mathrm{H}$ NMR spectrum of $5 \mathbf{v}\left(\mathrm{CD}_{3} \mathrm{COCD}_{3}, 600 \mathrm{MHz}, 23{ }^{\circ} \mathrm{C}\right)$. 


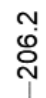
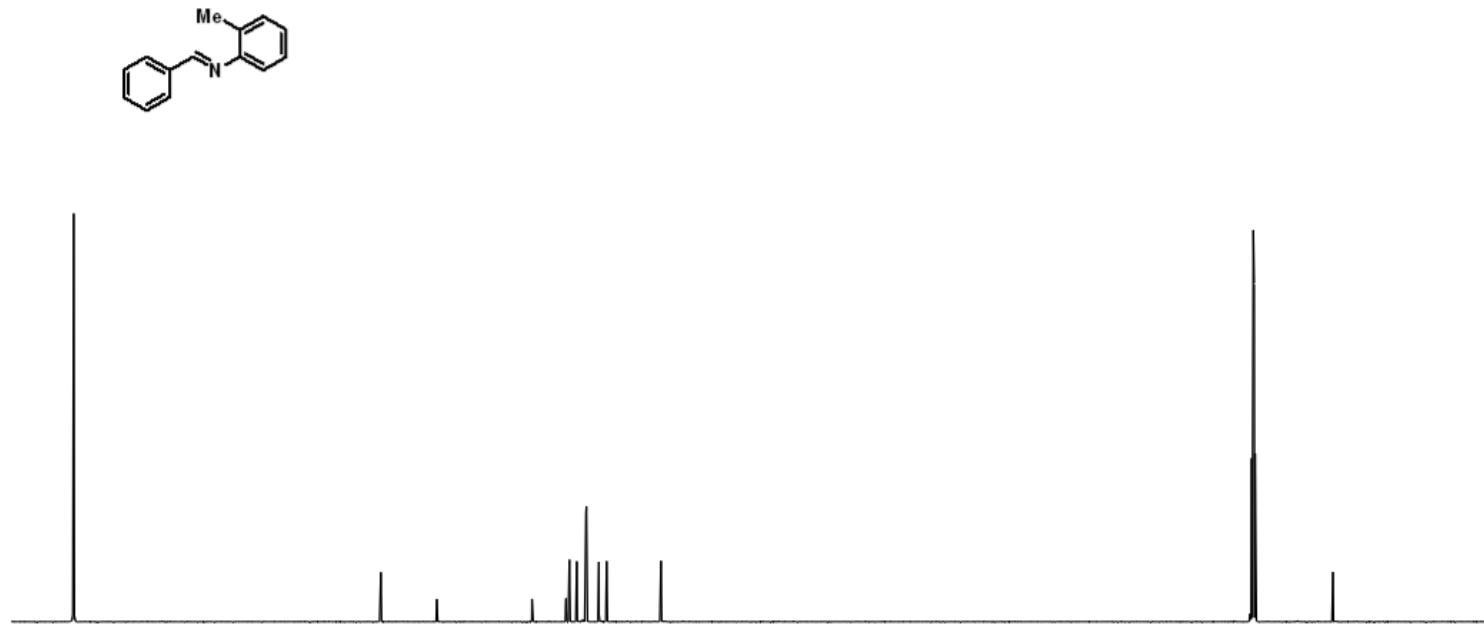

$\begin{array}{llllllllllllllllllllll}210 & 200 & 190 & 180 & 170 & 160 & 150 & 140 & 130 & 120 & \begin{array}{l}110 \\ \mathrm{f} 1(\mathrm{ppm})\end{array} & 100 & 90 & 80 & 70 & 60 & 50 & 40 & 30 & 20 & 10 & 0\end{array}$

Figure S53. ${ }^{13} \mathrm{C}$ NMR spectrum of $\mathbf{5 v}\left(\mathrm{CD}_{3} \mathrm{COCD}_{3}, 150 \mathrm{MHz}, 23{ }^{\circ} \mathrm{C}\right)$.

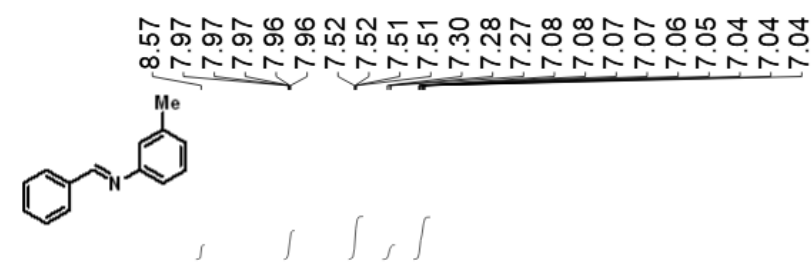

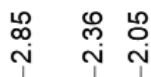

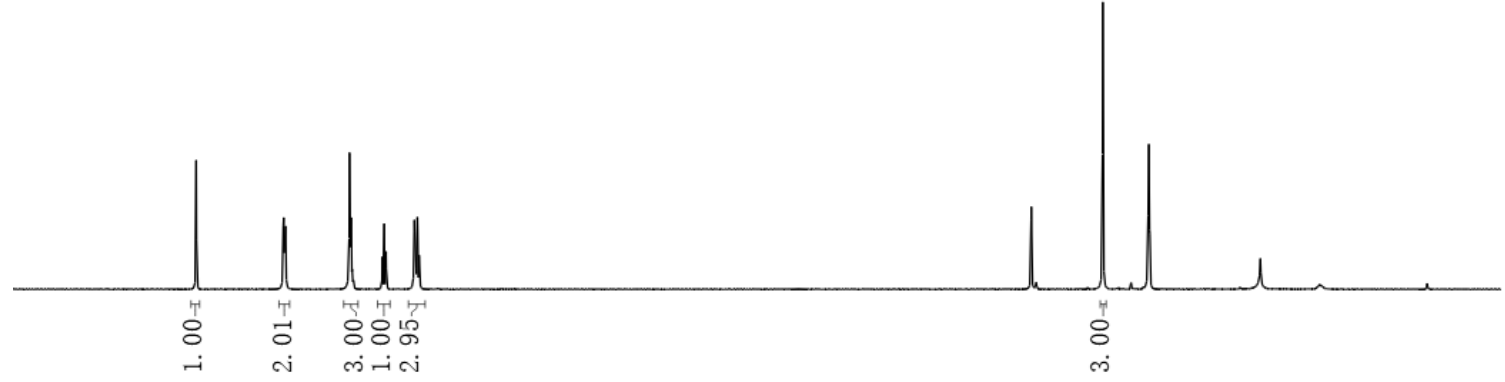

$\begin{array}{llllllllllllllllllll}9.5 & 9.0 & 8.5 & 8.0 & 7.5 & 7.0 & 6.5 & 6.0 & 5.5 & \begin{array}{l}5.0 \\ \mathrm{f} 1(\mathrm{ppm})\end{array} & 4.0 & 3.5 & 3.0 & 2.5 & 2.0 & 1.5 & 1.0 & 0.5 & 0.0\end{array}$

Figure S54. ${ }^{1} \mathrm{H}$ NMR spectrum of $5 \mathbf{w}\left(\mathrm{CD}_{3} \mathrm{COCD}_{3}, 600 \mathrm{MHz}, 23{ }^{\circ} \mathrm{C}\right)$. 


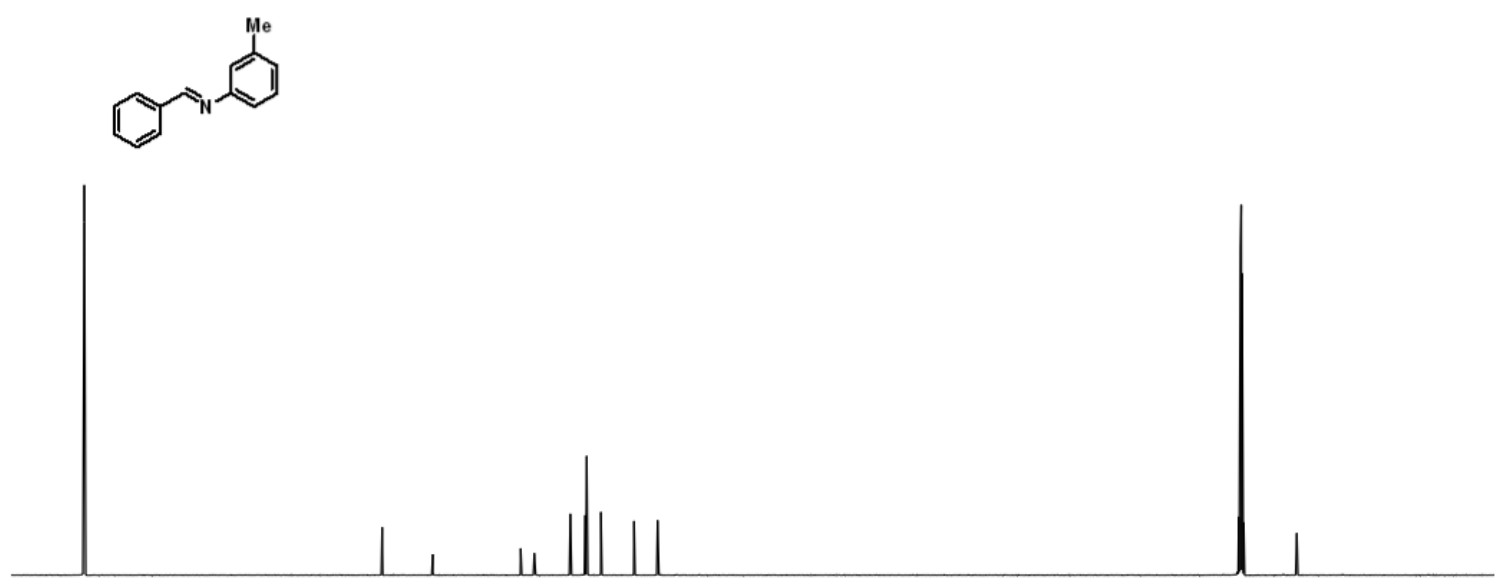

$\begin{array}{llllllllllllllllllllll}210 & 200 & 190 & 180 & 170 & 160 & 150 & 140 & 130 & 120 & \begin{array}{l}110 \\ \mathrm{f} 1(\mathrm{ppm})\end{array} & 90 & 80 & 70 & 60 & 50 & 40 & 30 & 20 & 10 & 0\end{array}$

Figure S55. ${ }^{13} \mathrm{C}$ NMR spectrum of $\mathbf{5 w}\left(\mathrm{CD}_{3} \mathrm{COCD}_{3}, 150 \mathrm{MHz}, 23{ }^{\circ} \mathrm{C}\right)$.
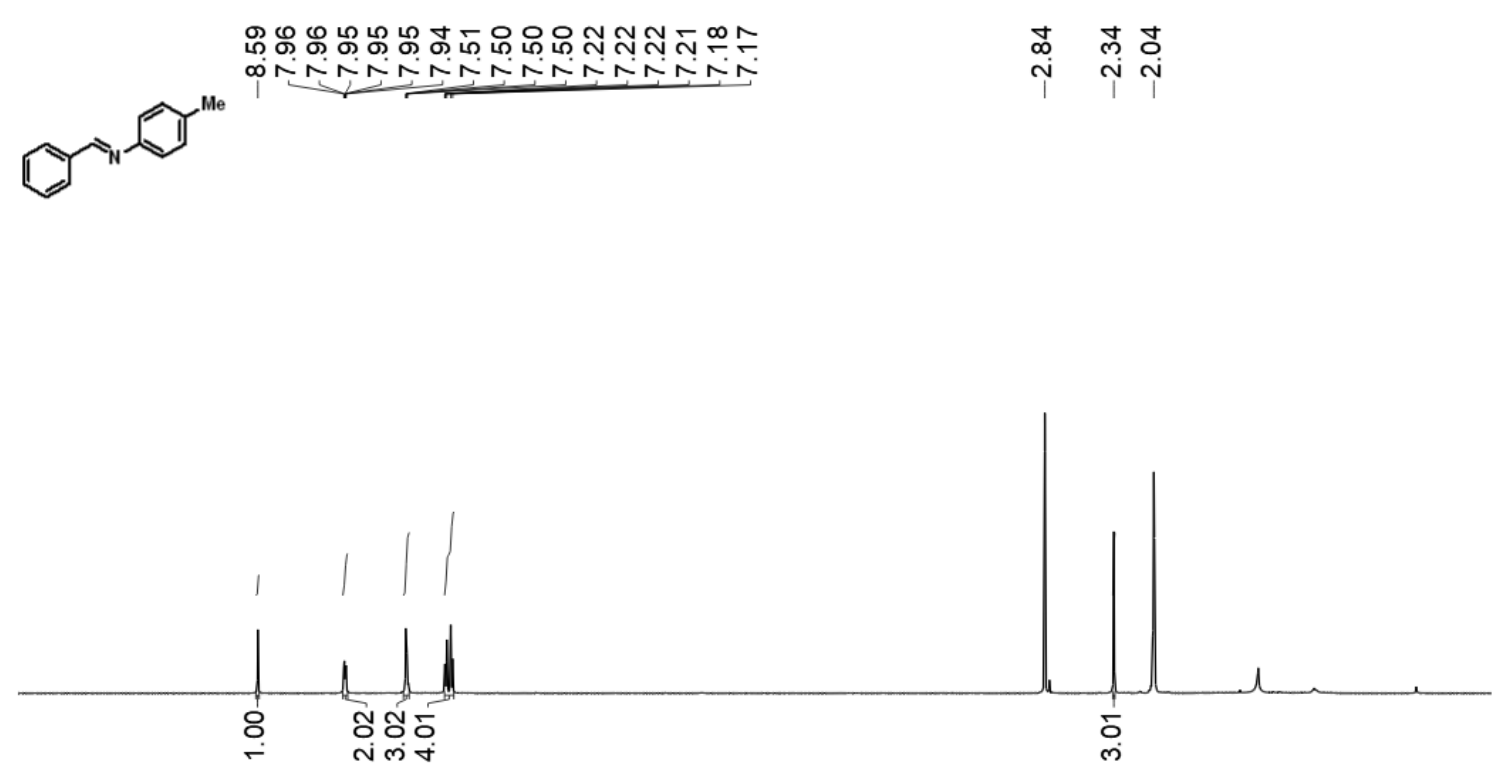

$\begin{array}{lllllllllllllllllllll}10.0 & 9.5 & 9.0 & 8.5 & 8.0 & 7.5 & 7.0 & 6.5 & 6.0 & 5.5 & \begin{array}{c}51 \\ \mathrm{f} 1 \mathrm{ppm})\end{array} & 4.5 & 4.0 & 3.5 & 3.0 & 2.5 & 2.0 & 1.5 & 1.0 & 0.5 & 0.0\end{array}$

Figure S56. ${ }^{1} \mathrm{H}$ NMR spectrum of $\mathbf{5 x}\left(\mathrm{CD}_{3} \mathrm{COCD}_{3}, 600 \mathrm{MHz}, 23{ }^{\circ} \mathrm{C}\right)$. 


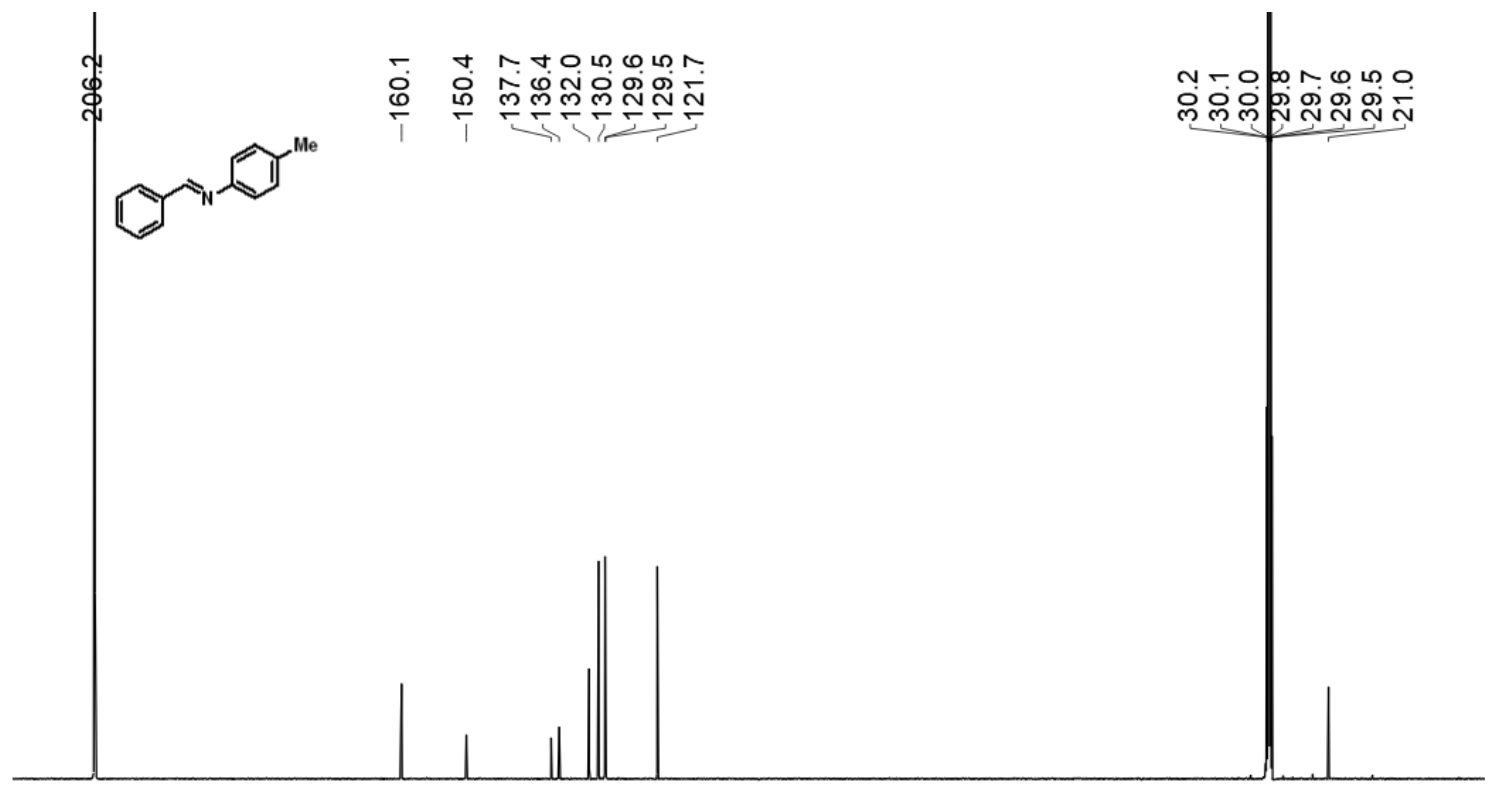

$\begin{array}{lllllllllllllllllllll}210 & 200 & 190 & 180 & 170 & 160 & 150 & 140 & 130 & 120 \begin{array}{c}110 \\ \mathrm{f} 1(\mathrm{ppm})\end{array} & 90 & 80 & 70 & 60 & 50 & 40 & 30 & 20 & 10 & 0\end{array}$

Figure S57. ${ }^{13} \mathrm{C}$ NMR spectrum of $\mathbf{5 x}\left(\mathrm{CD}_{3} \mathrm{COCD}_{3}, 150 \mathrm{MHz}, 23{ }^{\circ} \mathrm{C}\right)$.
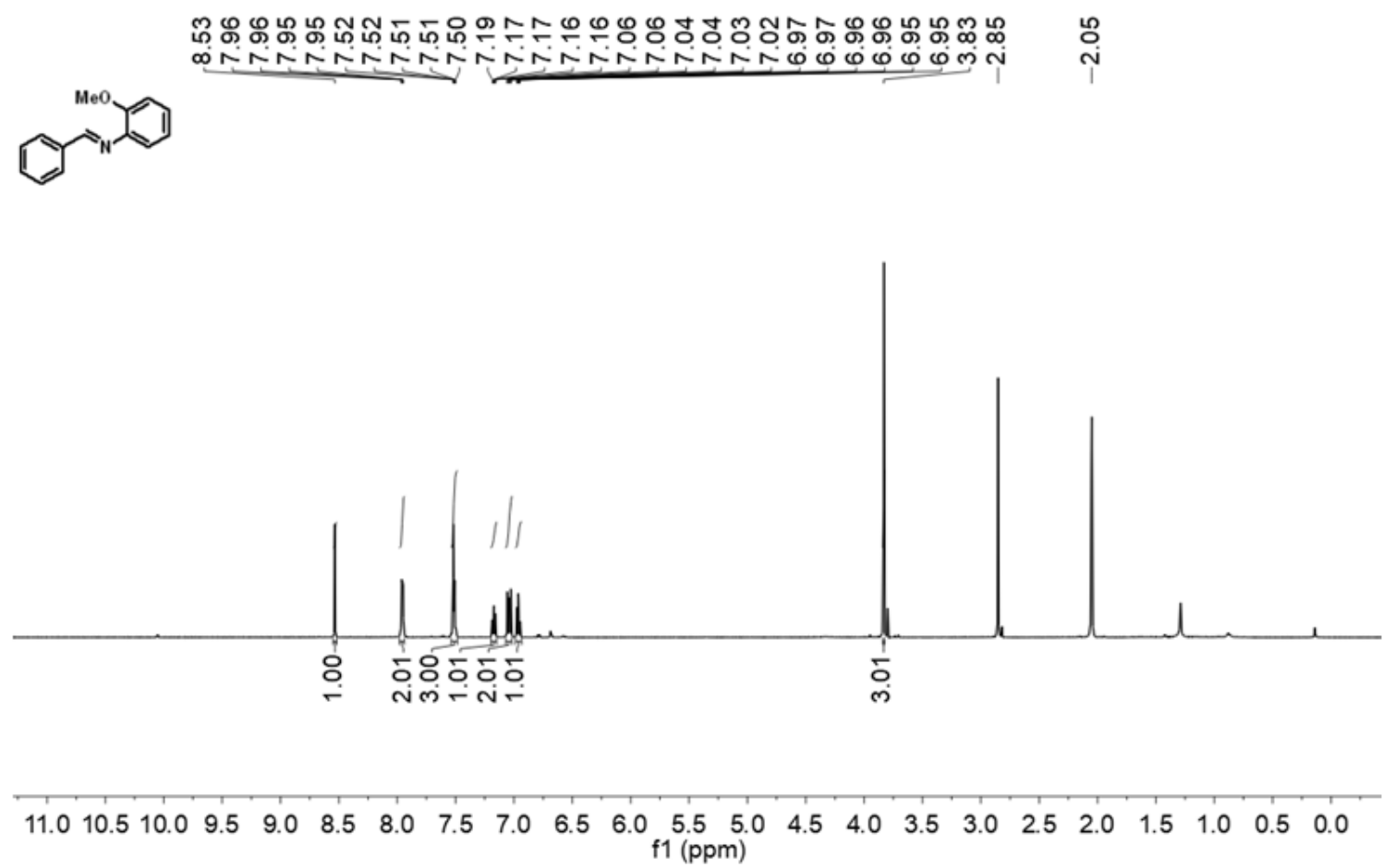

Figure S58. ${ }^{1} \mathrm{H}$ NMR spectrum of $\mathbf{5 y}\left(\mathrm{CD}_{3} \mathrm{COCD}_{3}, 600 \mathrm{MHz}, 23{ }^{\circ} \mathrm{C}\right)$. 


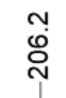

我<smiles>COc1ccccc1N=Cc1ccccc1</smiles>

ก- 0 on 0 in

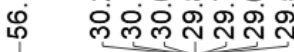

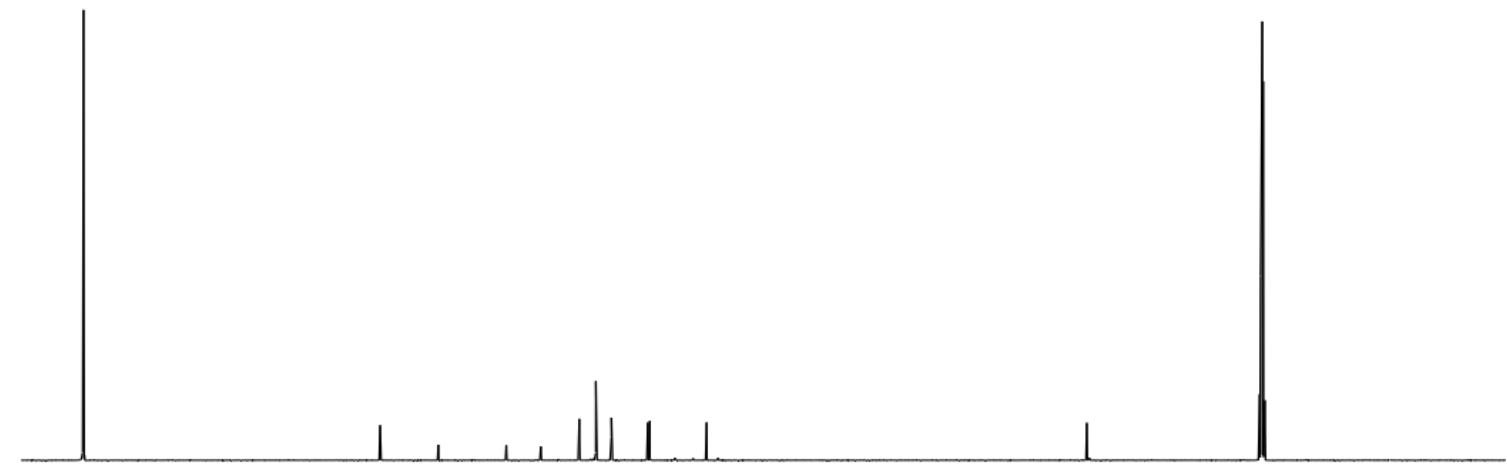

$\begin{array}{llllllllllllllllllllll}210 & 200 & 190 & 180 & 170 & 160 & 150 & 140 & 130 & 120 & \begin{array}{l}110 \\ \mathrm{f} 1(\mathrm{ppm})\end{array} & 90 & 80 & 70 & 60 & 50 & 40 & 30 & 20 & 10 & 0\end{array}$

Figure S59. ${ }^{13} \mathrm{C}$ NMR spectrum of $\mathbf{5 y}\left(\mathrm{CD}_{3} \mathrm{COCD}_{3}, 150 \mathrm{MHz}, 23{ }^{\circ} \mathrm{C}\right)$.
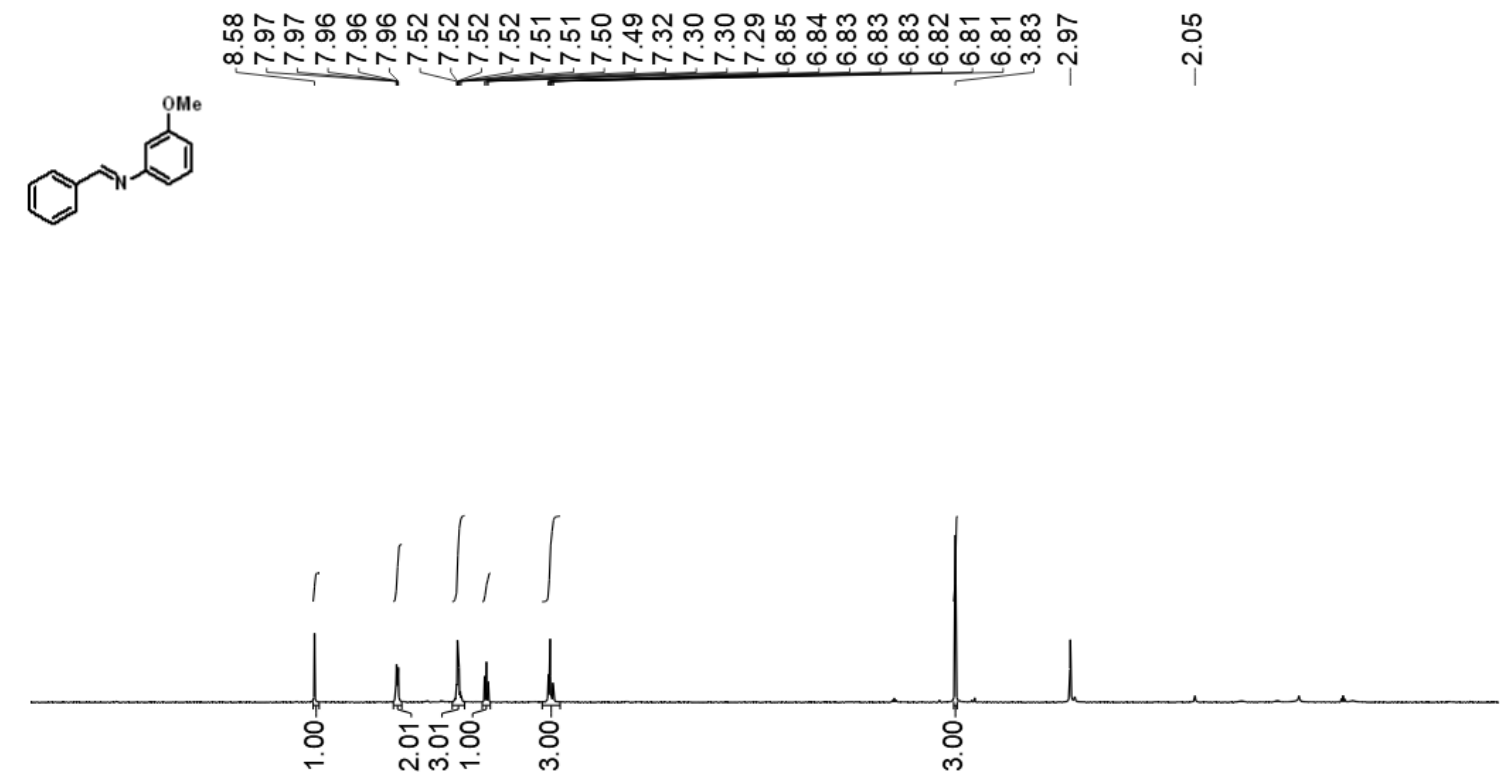

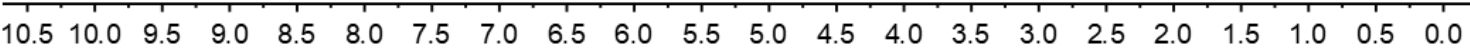

Figure S60. ${ }^{1} \mathrm{H}$ NMR spectrum of $\mathbf{5 z}\left(\mathrm{CD}_{3} \mathrm{COCD}_{3}, 600 \mathrm{MHz}, 23{ }^{\circ} \mathrm{C}\right)$. 

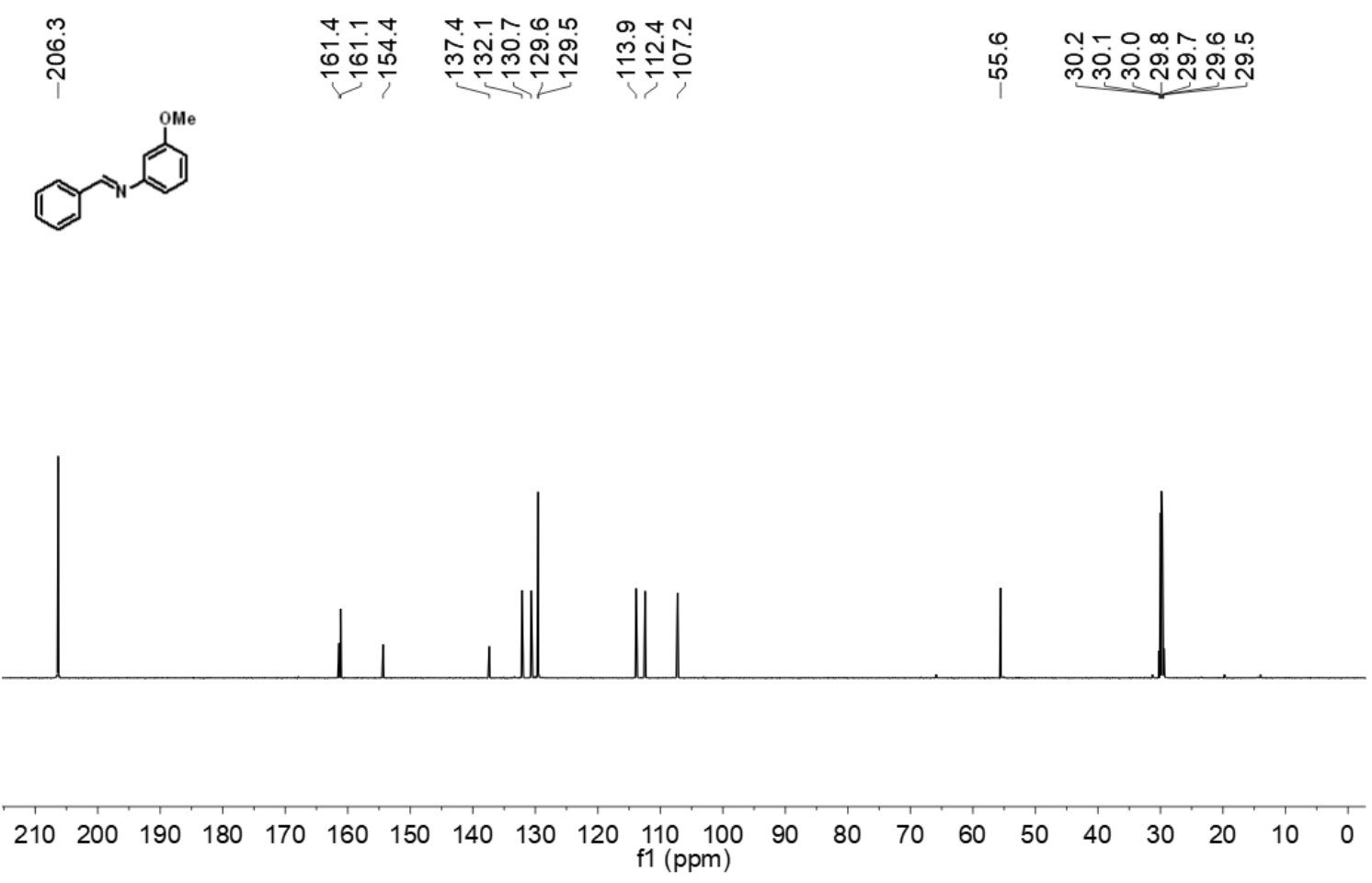

Figure S61. ${ }^{13} \mathrm{C}$ NMR spectrum of $\mathbf{5 z}\left(\mathrm{CD}_{3} \mathrm{COCD}_{3}, 150 \mathrm{MHz}, 23{ }^{\circ} \mathrm{C}\right)$.

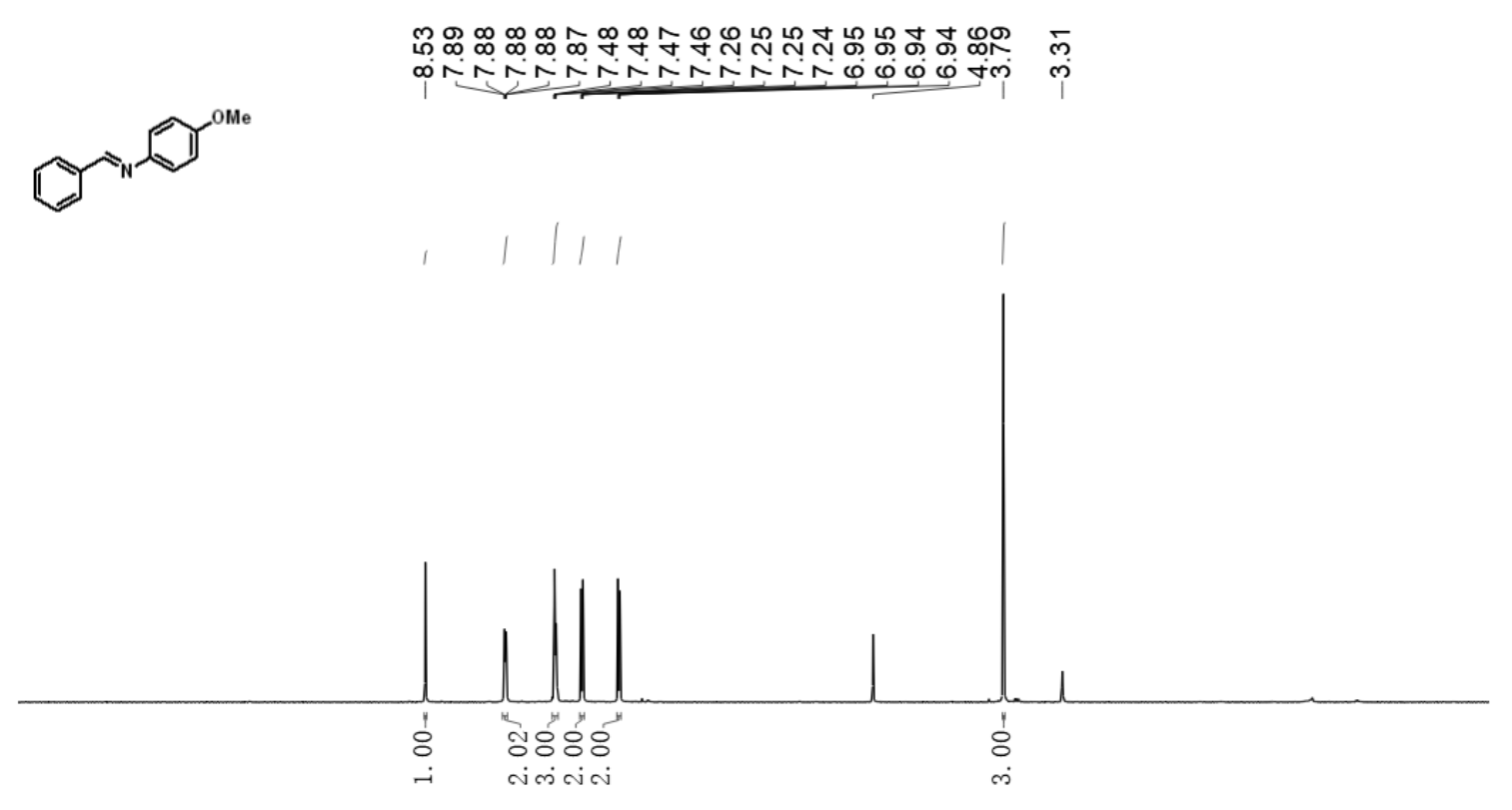
$\begin{array}{llllllllllllllllllllllllll}11.5 & 11.0 & 10.5 & 10.0 & 9.5 & 9.0 & 8.5 & 8.0 & 7.5 & 7.0 & 6.5 & 6.0 & 5.5 & 5.0 & 4.5 & 4.0 & 3.5 & 3.0 & 2.5 & 2.0 & 1.5 & 1.0 & 0.5 & 0.0\end{array}$

Figure S62. ${ }^{1} \mathrm{H}$ NMR spectrum of $\mathbf{5 a a}\left(\mathrm{CD}_{3} \mathrm{OD}, 600 \mathrm{MHz}, 23{ }^{\circ} \mathrm{C}\right)$. 


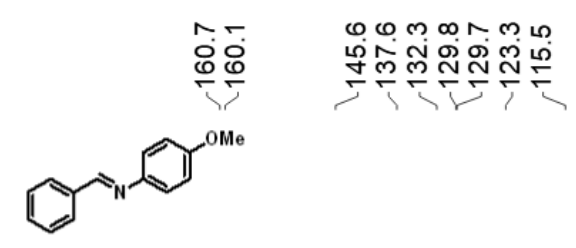

कำ

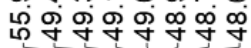

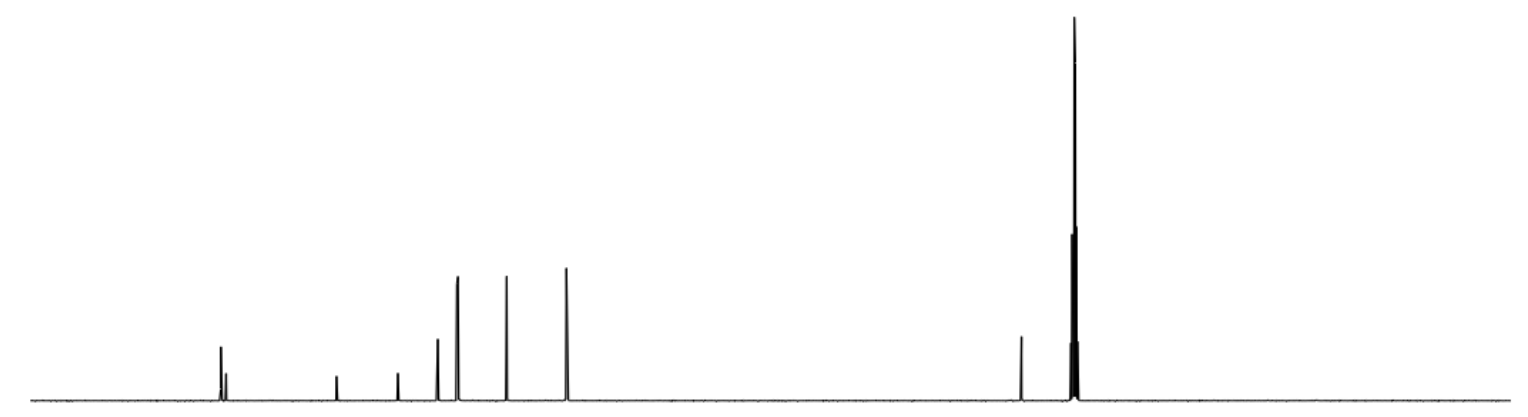

$\begin{array}{llllllllllllllllll}180 & 170 & 160 & 150 & 140 & 130 & 120 & 110 & 100 \underset{\mathrm{f} 1}{90}(\mathrm{ppm}) & 80 & 70 & 60 & 50 & 40 & 30 & 20 & 10 & 0\end{array}$

Figure S63. ${ }^{13} \mathrm{C}$ NMR spectrum of $\mathbf{5 a a}\left(\mathrm{CD}_{3} \mathrm{OD}, 150 \mathrm{MHz}, 23{ }^{\circ} \mathrm{C}\right)$.
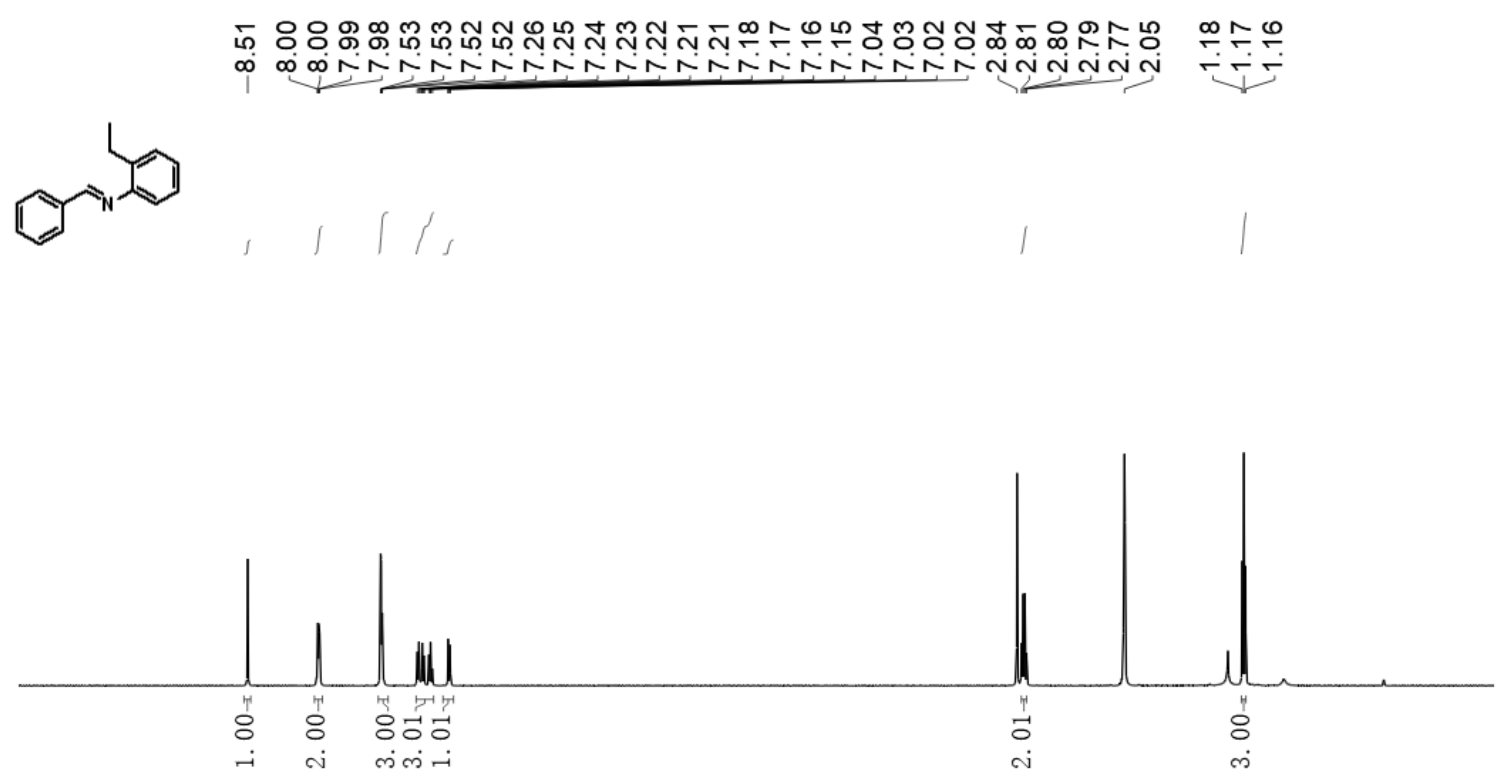

$\begin{array}{llllllllllllllllllllllll}10.0 & 9.5 & 9.0 & 8.5 & 8.0 & 7.5 & 7.0 & 6.5 & 6.0 & 5.5 & \begin{array}{l}5.0 \\ \mathrm{f} 1(\mathrm{ppm})\end{array} & 4.5 & 4.0 & 3.5 & 3.0 & 2.5 & 2.0 & 1.5 & 1.0 & 0.5 & 0.0 & -0.5\end{array}$

Figure S64. ${ }^{1} \mathrm{H}$ NMR spectrum of $\mathbf{5 a b}\left(\mathrm{CD}_{3} \mathrm{COCD}_{3}, 600 \mathrm{MHz}, 23{ }^{\circ} \mathrm{C}\right)$. 


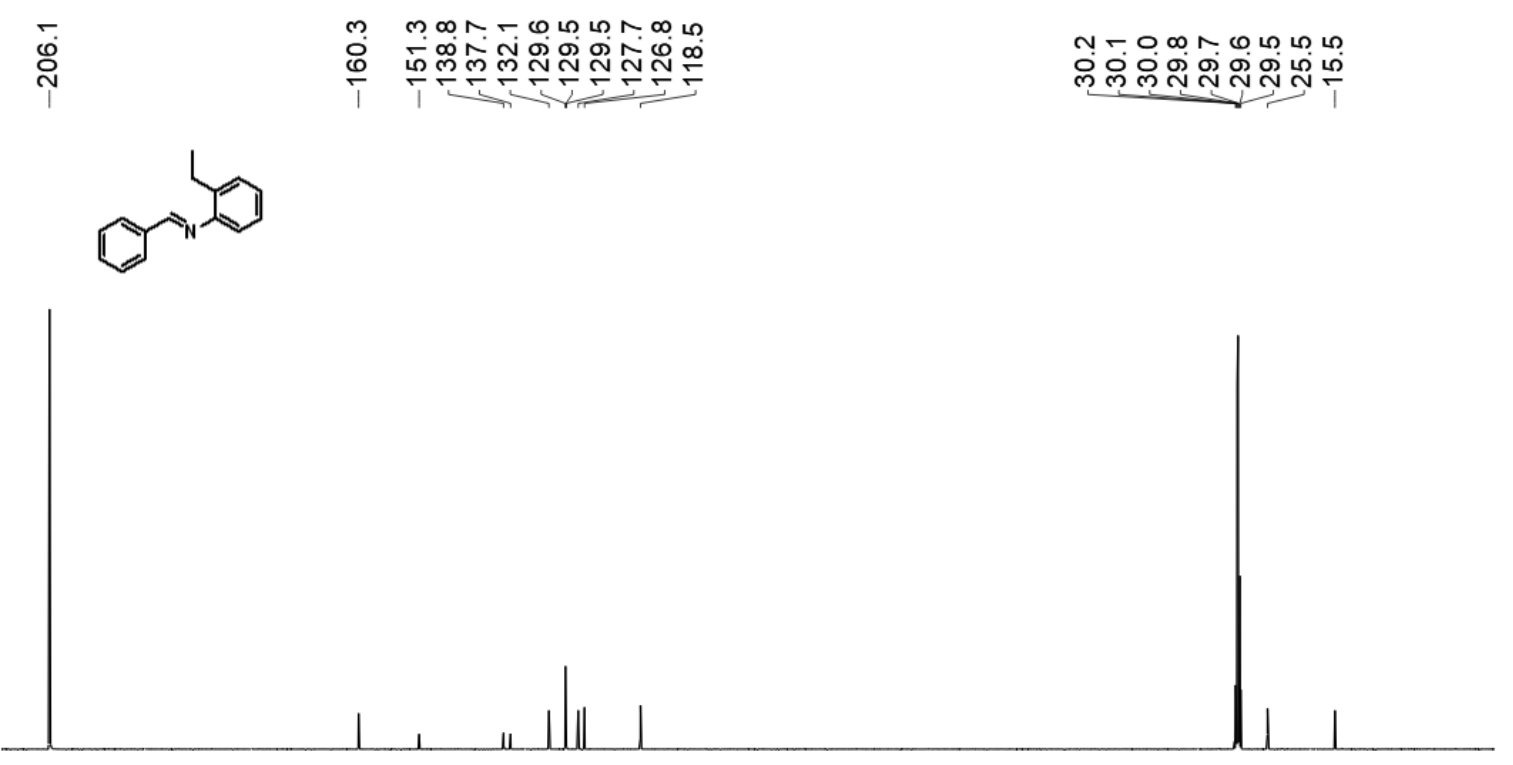

$\begin{array}{llllllllllllllllllllll}210 & 200 & 190 & 180 & 170 & 160 & 150 & 140 & 130 & 120 & \begin{array}{c}110 \\ \mathrm{f} 1(\mathrm{ppm})\end{array} & 100 & 90 & 80 & 70 & 60 & 50 & 40 & 30 & 20 & 10 & 0\end{array}$

Figure S65. ${ }^{13} \mathrm{C}$ NMR spectrum of $\mathbf{5 a b}\left(\mathrm{CD}_{3} \mathrm{COCD}_{3}, 150 \mathrm{MHz}, 23{ }^{\circ} \mathrm{C}\right)$.
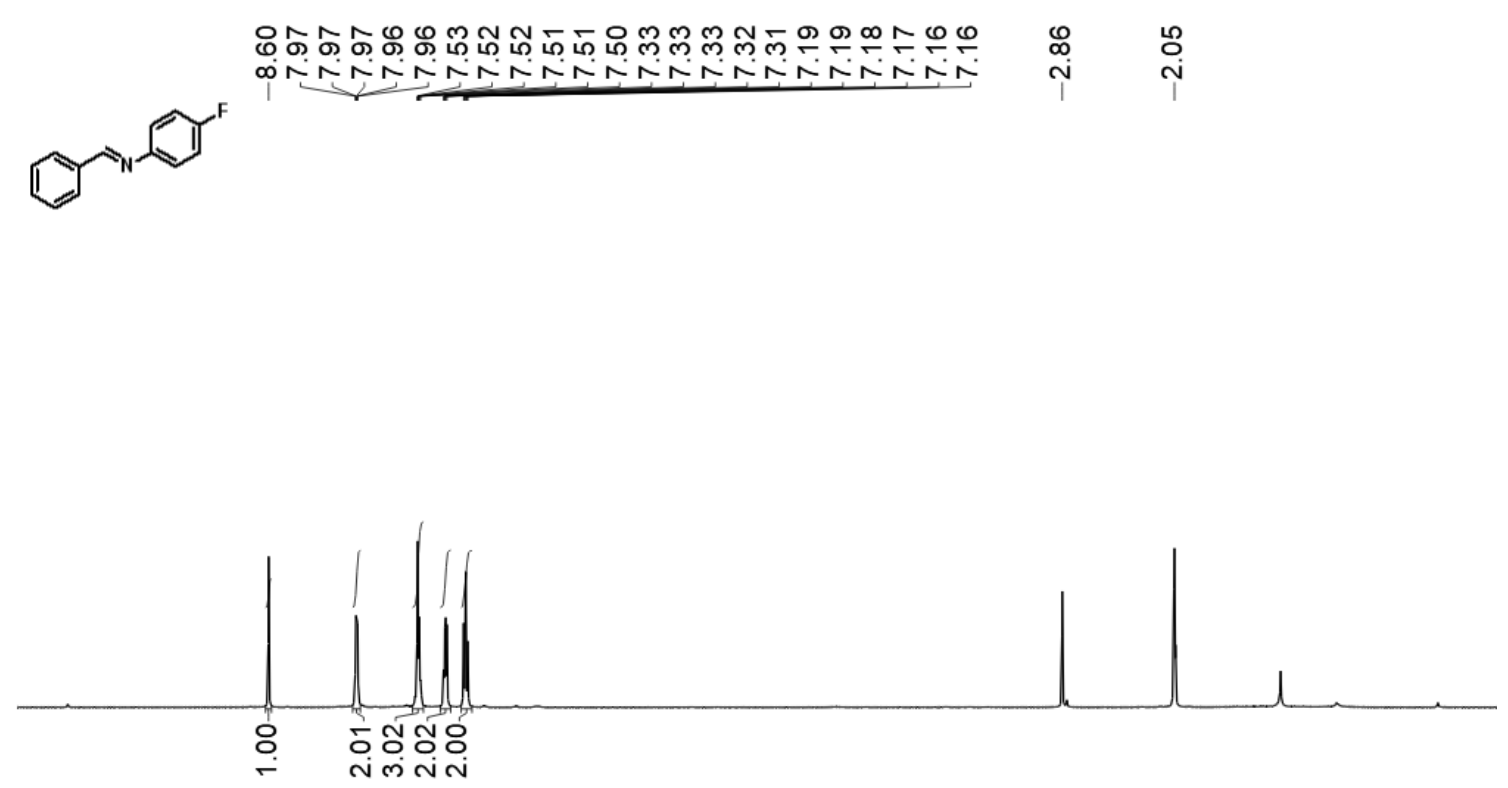

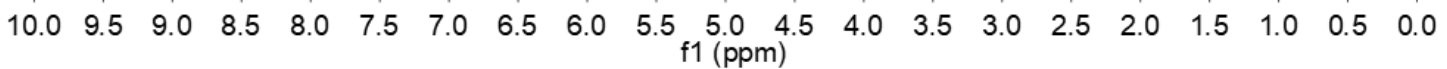

Figure S66. ${ }^{1} \mathrm{H}$ NMR spectrum of $5 \mathbf{a c}\left(\mathrm{CD}_{3} \mathrm{COCD}_{3}, 600 \mathrm{MHz}, 23{ }^{\circ} \mathrm{C}\right)$. 


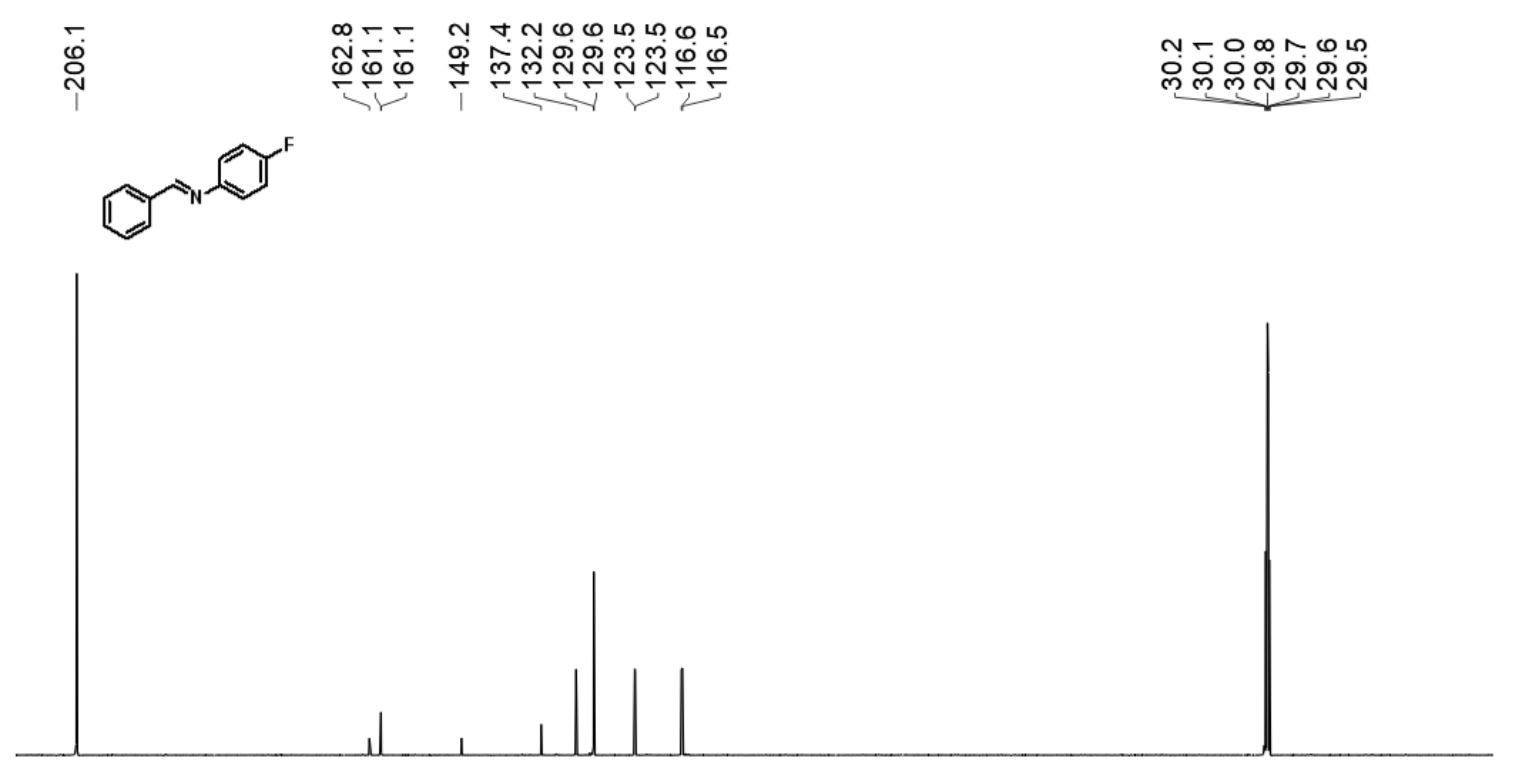

$\begin{array}{llllllllllllllllllllll}210 & 200 & 190 & 180 & 170 & 160 & 150 & 140 & 130 & 120 & \begin{array}{c}110 \\ \mathrm{f} 1(\mathrm{ppm})\end{array} & 90 & 80 & 70 & 60 & 50 & 40 & 30 & 20 & 10 & 0\end{array}$

Figure S67. ${ }^{13} \mathrm{C}$ NMR spectrum of $5 \mathbf{a c}\left(\mathrm{CD}_{3} \mathrm{COCD}_{3}, 150 \mathrm{MHz}, 23{ }^{\circ} \mathrm{C}\right)$.
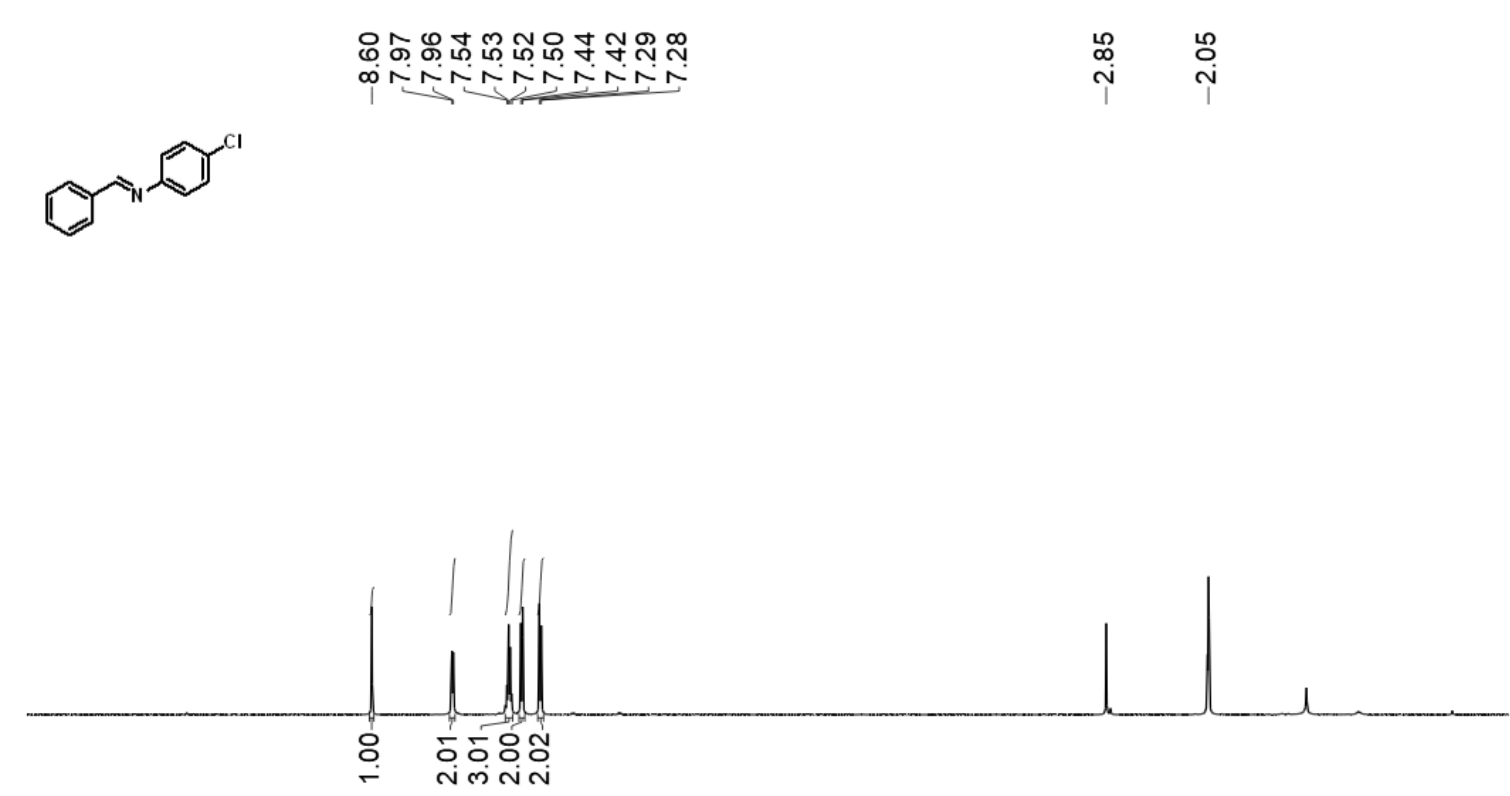

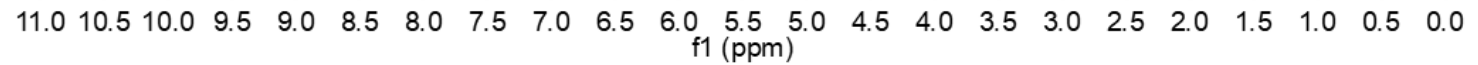

Figure S68. ${ }^{1} \mathrm{H}$ NMR spectrum of $\mathbf{5 a d}\left(\mathrm{CD}_{3} \mathrm{COCD}_{3}, 600 \mathrm{MHz}, 23{ }^{\circ} \mathrm{C}\right)$. 
¿্ঠ்<smiles>Clc1ccc(N=Cc2ccccc2)cc1</smiles>

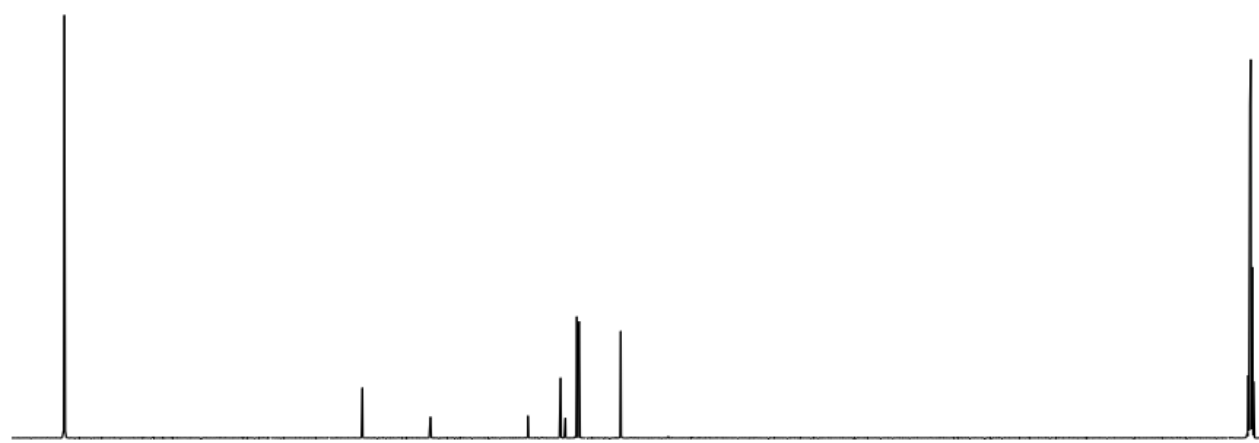

$\begin{array}{llllllllllllllllllllll}210 & 200 & 190 & 180 & 170 & 160 & 150 & 140 & 130 & 120 & \begin{array}{c}110 \\ \mathrm{f} 1(\mathrm{ppm})\end{array} & 100 & 90 & 80 & 70 & 60 & 50 & 40 & 30 & 20 & 10 & 0\end{array}$

Figure S69. ${ }^{13} \mathrm{C}$ NMR spectrum of $5 \mathbf{a d}\left(\mathrm{CD}_{3} \mathrm{COCD}_{3}, 150 \mathrm{MHz}, 23{ }^{\circ} \mathrm{C}\right)$.

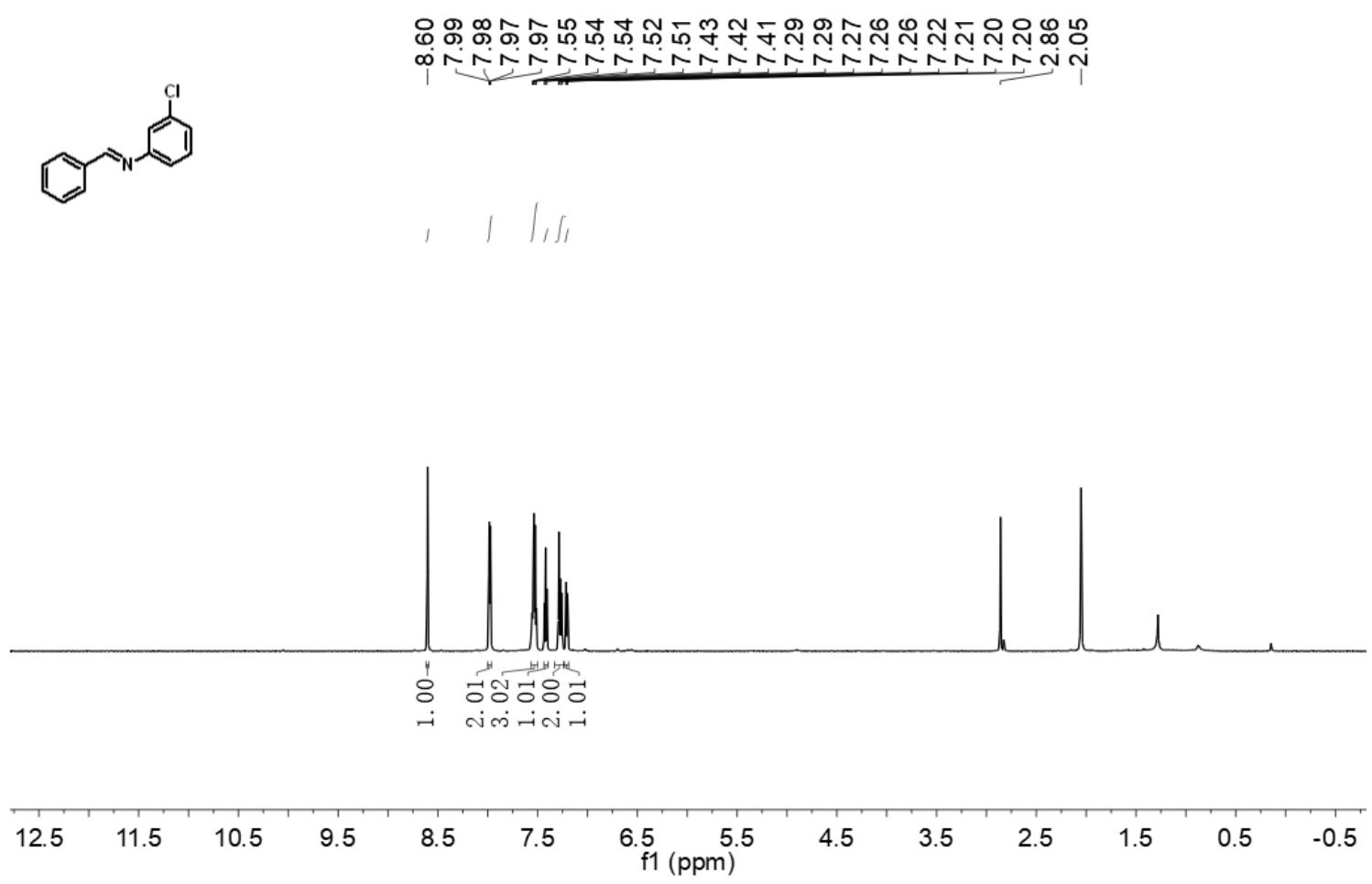

Figure S70. ${ }^{1} \mathrm{H}$ NMR spectrum of 5ae $\left(\mathrm{CD}_{3} \mathrm{COCD}_{3}, 600 \mathrm{MHz}, 23{ }^{\circ} \mathrm{C}\right)$. 
$\underset{\grave{i}}{\Gamma}$<smiles>Clc1cccc(N=Cc2ccccc2)c1</smiles>

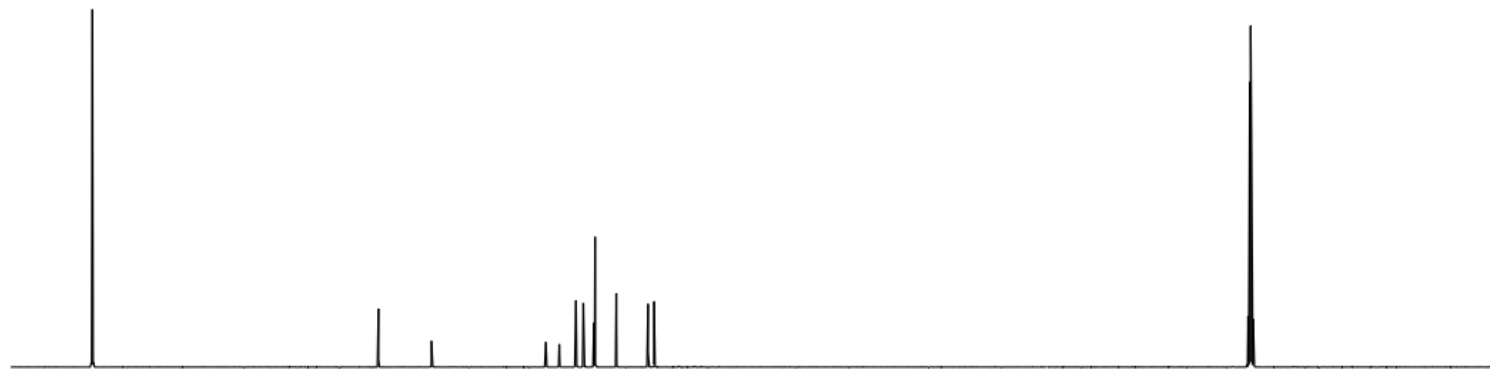

$\begin{array}{llllllllllllllllllllll}210 & 200 & 190 & 180 & 170 & 160 & 150 & 140 & 130 & 120 & \begin{array}{c}110 \\ \mathrm{f} 1(\mathrm{ppm})\end{array} & 90 & 80 & 70 & 60 & 50 & 40 & 30 & 20 & 10 & 0\end{array}$

Figure S71. ${ }^{13} \mathrm{C}$ NMR spectrum of $5 \mathbf{a e}\left(\mathrm{CD}_{3} \mathrm{COCD}_{3}, 150 \mathrm{MHz}, 23{ }^{\circ} \mathrm{C}\right)$.

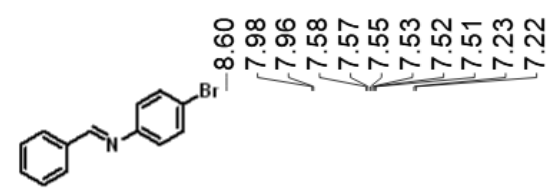

$\stackrel{\infty}{\stackrel{\infty}{i}} \stackrel{\stackrel{\infty}{i}}{\uparrow}$

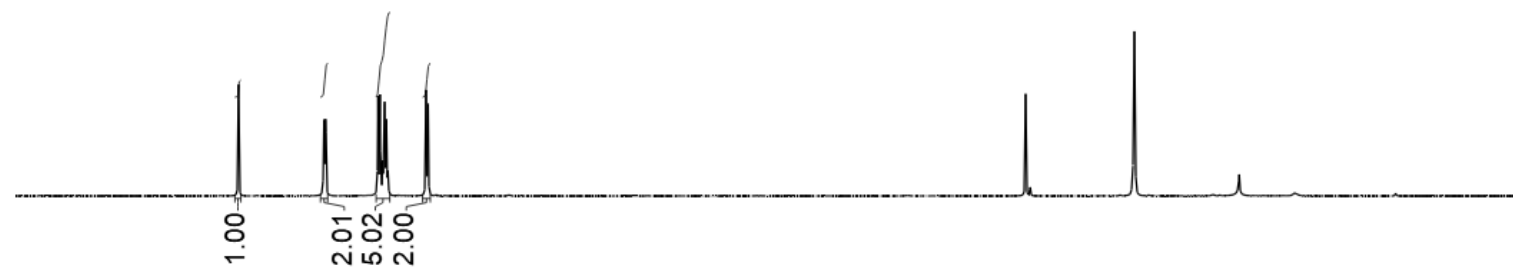

$\begin{array}{llllllllllllllllllllll}10.0 & 9.5 & 9.0 & 8.5 & 8.0 & 7.5 & 7.0 & 6.5 & 6.0 & 5.5 & \begin{array}{l}5.0 \\ \mathrm{f} 1(\mathrm{ppm})\end{array} & 4.0 & 3.5 & 3.0 & 2.5 & 2.0 & 1.5 & 1.0 & 0.5 & 0.0 & -0.5\end{array}$

Figure S72. ${ }^{1} \mathrm{H} \mathrm{NMR}$ spectrum of 5 af $\left(\mathrm{CD}_{3} \mathrm{COCD}_{3}, 600 \mathrm{MHz}, 23{ }^{\circ} \mathrm{C}\right)$. 


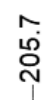

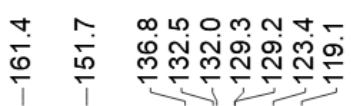

$\infty \sin m-0$

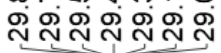<smiles>Brc1ccc(N=Cc2ccccc2)cc1</smiles>

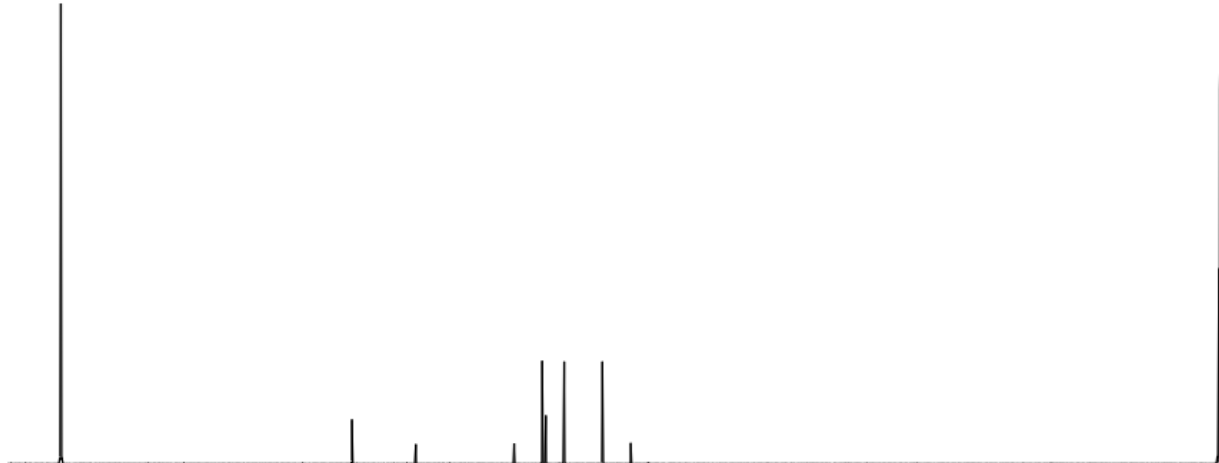

$\begin{array}{llllllllllllllllllllll}210 & 200 & 190 & 180 & 170 & 160 & 150 & 140 & 130 & 120 & \begin{array}{c}110 \\ \mathrm{f} 1(\mathrm{ppm})\end{array} & 90 & 80 & 70 & 60 & 50 & 40 & 30 & 20 & 10 & 0\end{array}$

Figure S73. ${ }^{13} \mathrm{C}$ NMR spectrum of 5 af $\left(\mathrm{CD}_{3} \mathrm{COCD}_{3}, 150 \mathrm{MHz}, 23{ }^{\circ} \mathrm{C}\right)$.

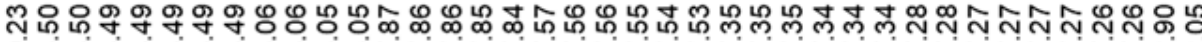

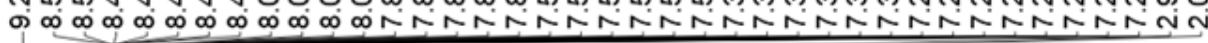<smiles>C(=Nc1cccnn1)c1ccccc1</smiles>

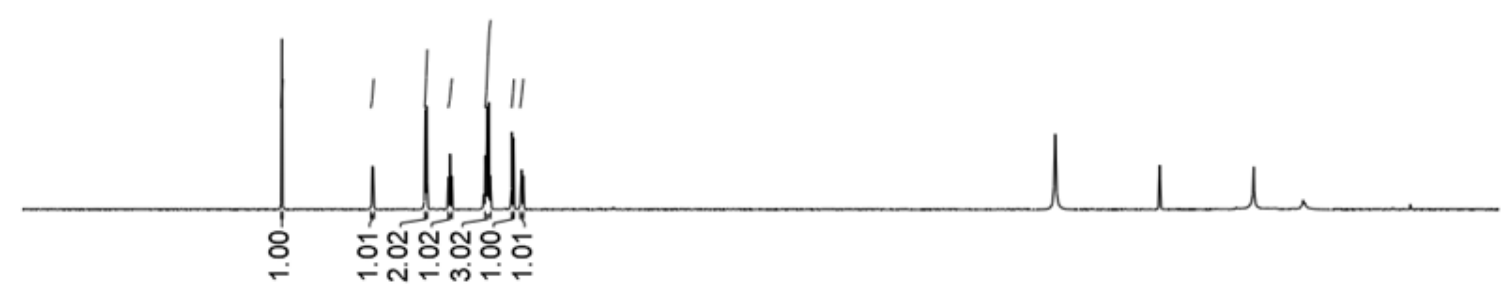

$\begin{array}{llllllllllllllllllllllll}11.0 & 10.5 & 10.0 & 9.5 & 9.0 & 8.5 & 8.0 & 7.5 & 7.0 & 6.5 & 6.0 & 5.5 & 5.0 & 4.5 & 4.0 & 3.5 & 3.0 & 2.5 & 2.0 & 1.5 & 1.0 & 0.5 & 0.0 & -0.5\end{array}$

Figure S74. ${ }^{1} \mathrm{H}$ NMR spectrum of $\mathbf{5 a g}\left(\mathrm{CD}_{3} \mathrm{COCD}_{3}, 600 \mathrm{MHz}, 23{ }^{\circ} \mathrm{C}\right)$. 
ํㅜㅁ

ก⿻

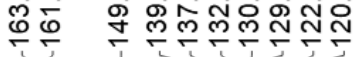

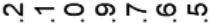

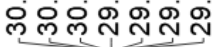<smiles></smiles>

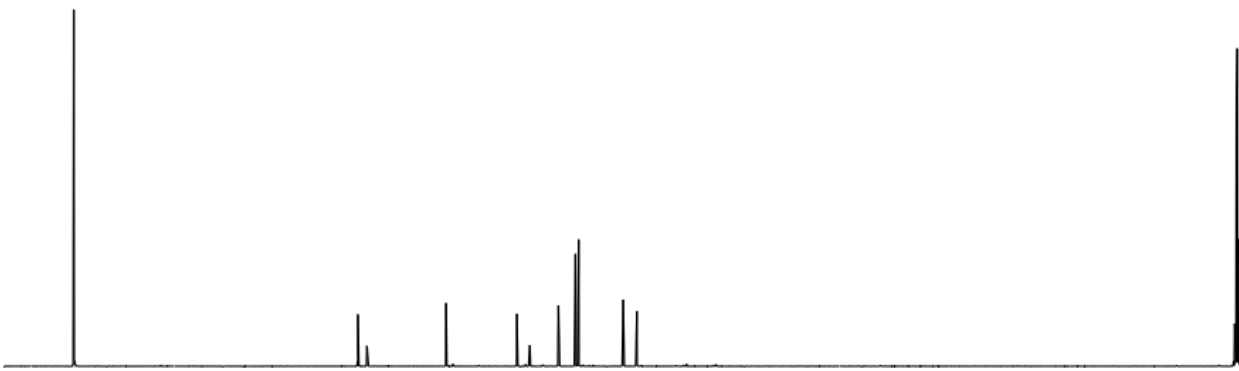

$\begin{array}{llllllllllllllllllllll}210 & 200 & 190 & 180 & 170 & 160 & 150 & 140 & 130 & 120 & 110 & 100 & 90 & 80 & 70 & 60 & 50 & 40 & 30 & 20 & 10 & 0\end{array}$

Figure S75. ${ }^{13} \mathrm{C}$ NMR spectrum of $\mathbf{5 a g}\left(\mathrm{CD}_{3} \mathrm{COCD}_{3}, 150 \mathrm{MHz}, 23{ }^{\circ} \mathrm{C}\right)$.

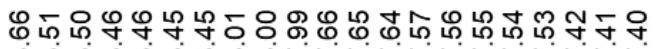

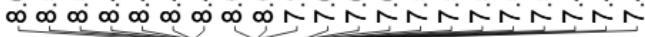

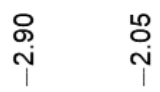<smiles>C(=Nc1ccccc1)c1ccccc1</smiles>

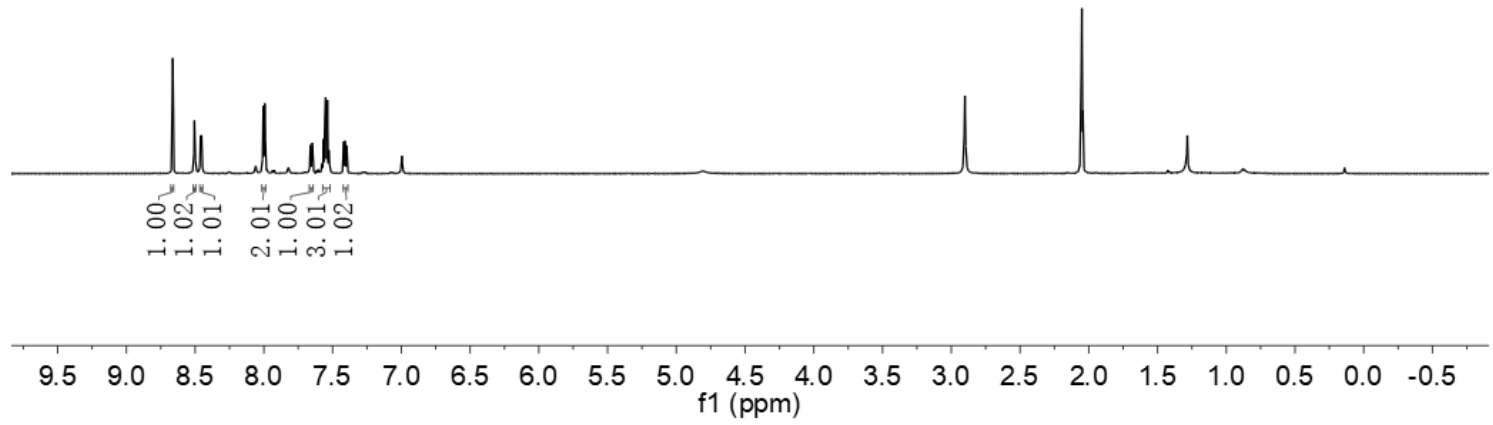

Figure S76. ${ }^{1} \mathrm{H}$ NMR spectrum of $\mathbf{5 a h}\left(\mathrm{CD}_{3} \mathrm{COCD}_{3}, 600 \mathrm{MHz}, 23{ }^{\circ} \mathrm{C}\right)$. 
$\stackrel{N}{\stackrel{N}{*}}$

ฯ $\varphi \circ \infty-\varphi \infty \Lambda-\varphi$

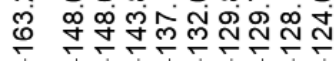

N-O 0 N 0 L

อิ
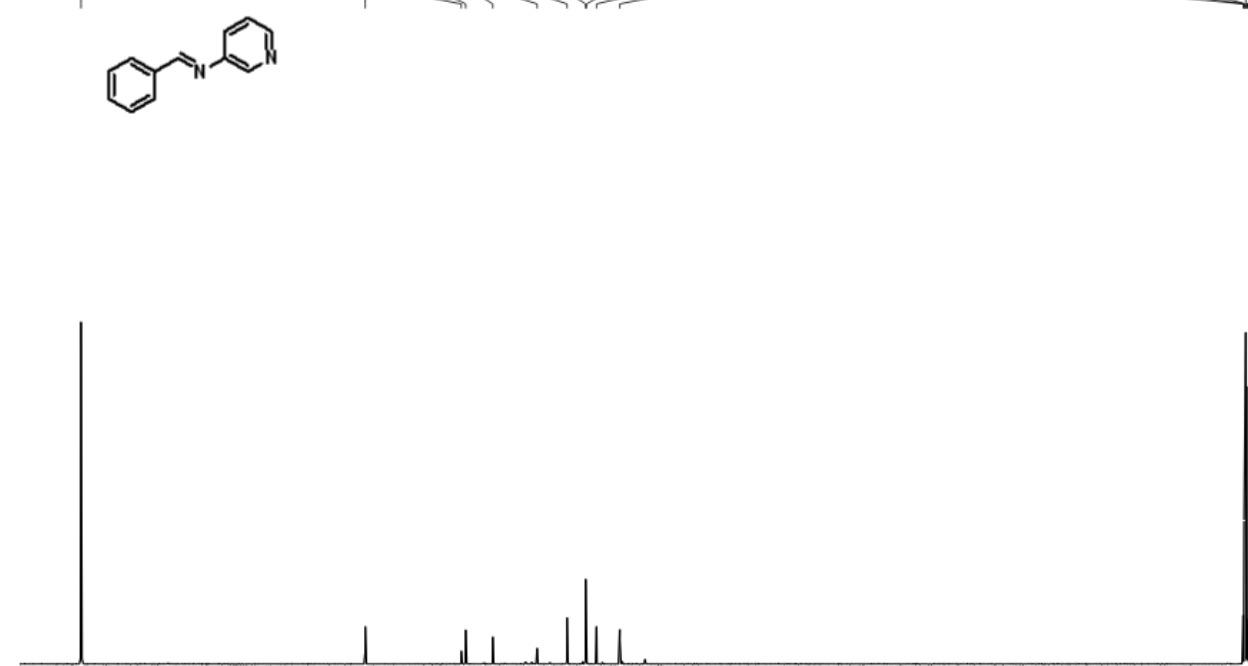

$\begin{array}{llllllllllllllllllllll}210 & 200 & 190 & 180 & 170 & 160 & 150 & 140 & 130 & 120 & \begin{array}{c}110 \\ \mathrm{f} 1(\mathrm{ppm})\end{array}\end{array}$

Figure S77. ${ }^{13} \mathrm{C}$ NMR spectrum of $\mathbf{5 a h}\left(\mathrm{CD}_{3} \mathrm{COCD}_{3}, 150 \mathrm{MHz}, 23{ }^{\circ} \mathrm{C}\right)$.

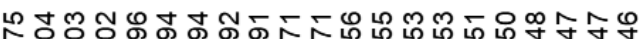

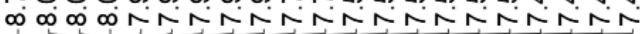

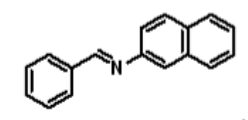

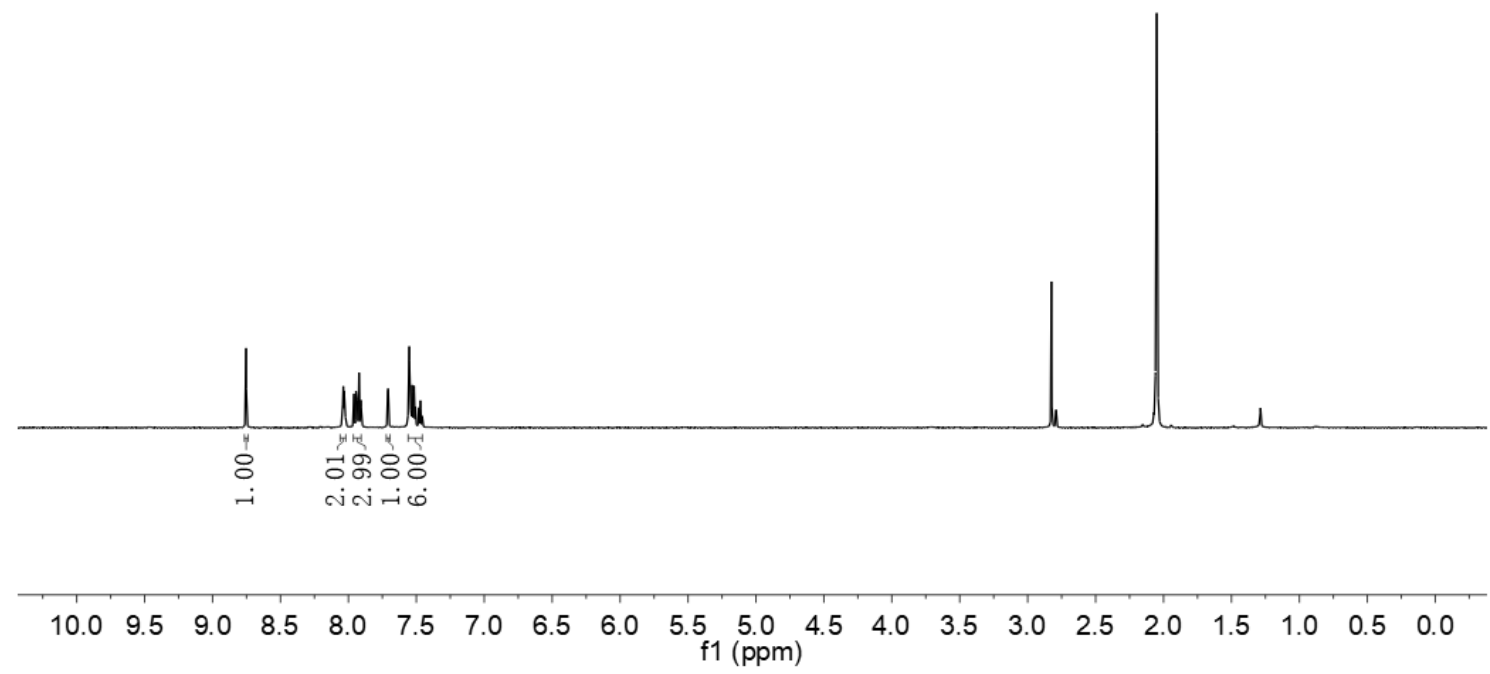

Figure S78. ${ }^{1} \mathrm{H}$ NMR spectrum of $\mathbf{5 a i}\left(\mathrm{CD}_{3} \mathrm{COCD}_{3}, 600 \mathrm{MHz}, 23^{\circ} \mathrm{C}\right)$. 


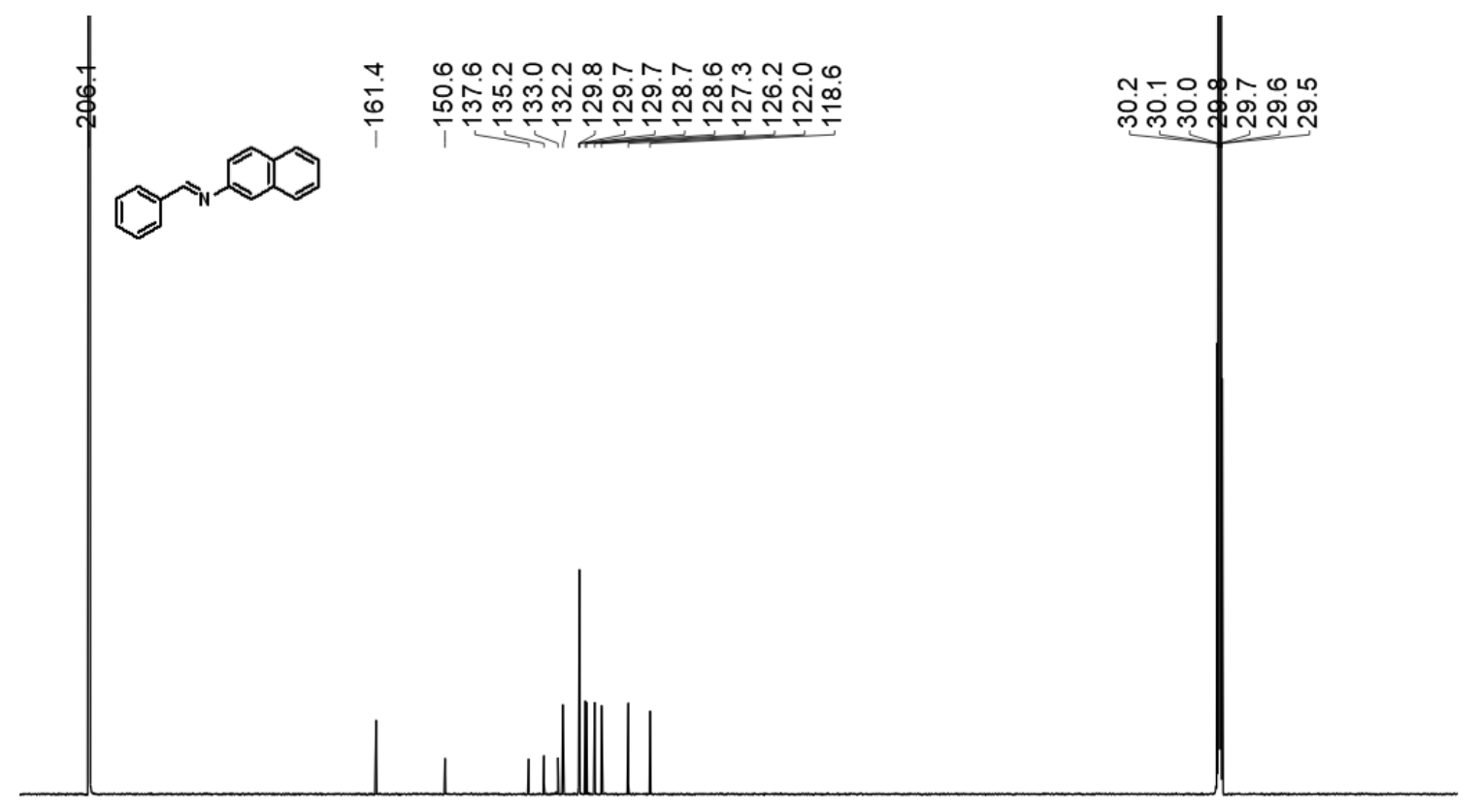

$\begin{array}{llllllllllllllllllllll}210 & 200 & 190 & 180 & 170 & 160 & 150 & 140 & 130 & 120 & \begin{array}{l}110 \\ \mathrm{f} 1(\mathrm{ppm})\end{array} & 90 & 80 & 70 & 60 & 50 & 40 & 30 & 20 & 10 & 0\end{array}$

Figure S79. ${ }^{13} \mathrm{C}$ NMR spectrum of 5 ai $\left(\mathrm{CD}_{3} \mathrm{COCD}_{3}, 150 \mathrm{MHz}, 23{ }^{\circ} \mathrm{C}\right)$.

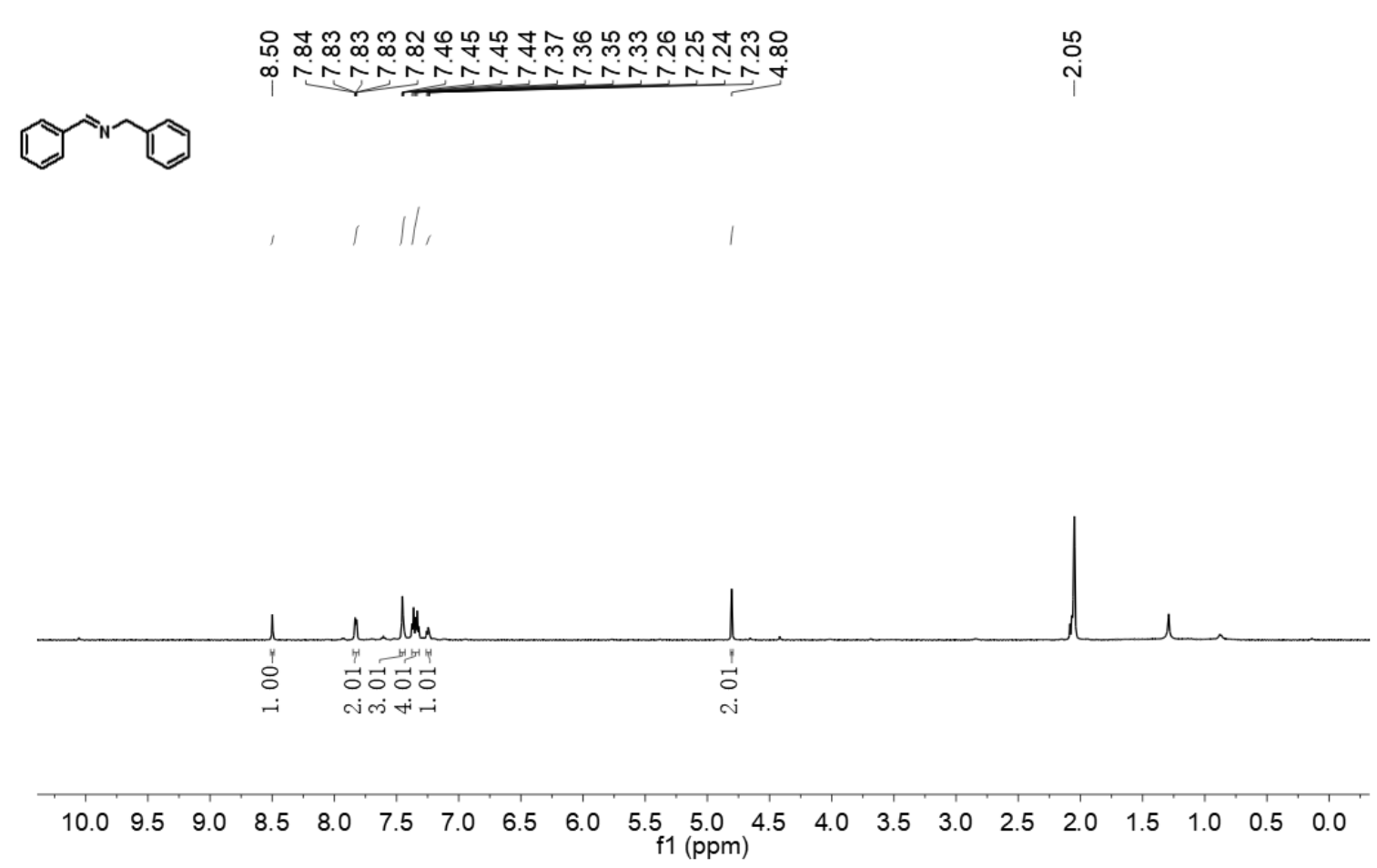

Figure S80. ${ }^{1} \mathrm{H}$ NMR spectrum of $\mathbf{5 a j}\left(\mathrm{CD}_{3} \mathrm{COCD}_{3}, 600 \mathrm{MHz}, 23^{\circ} \mathrm{C}\right)$. 


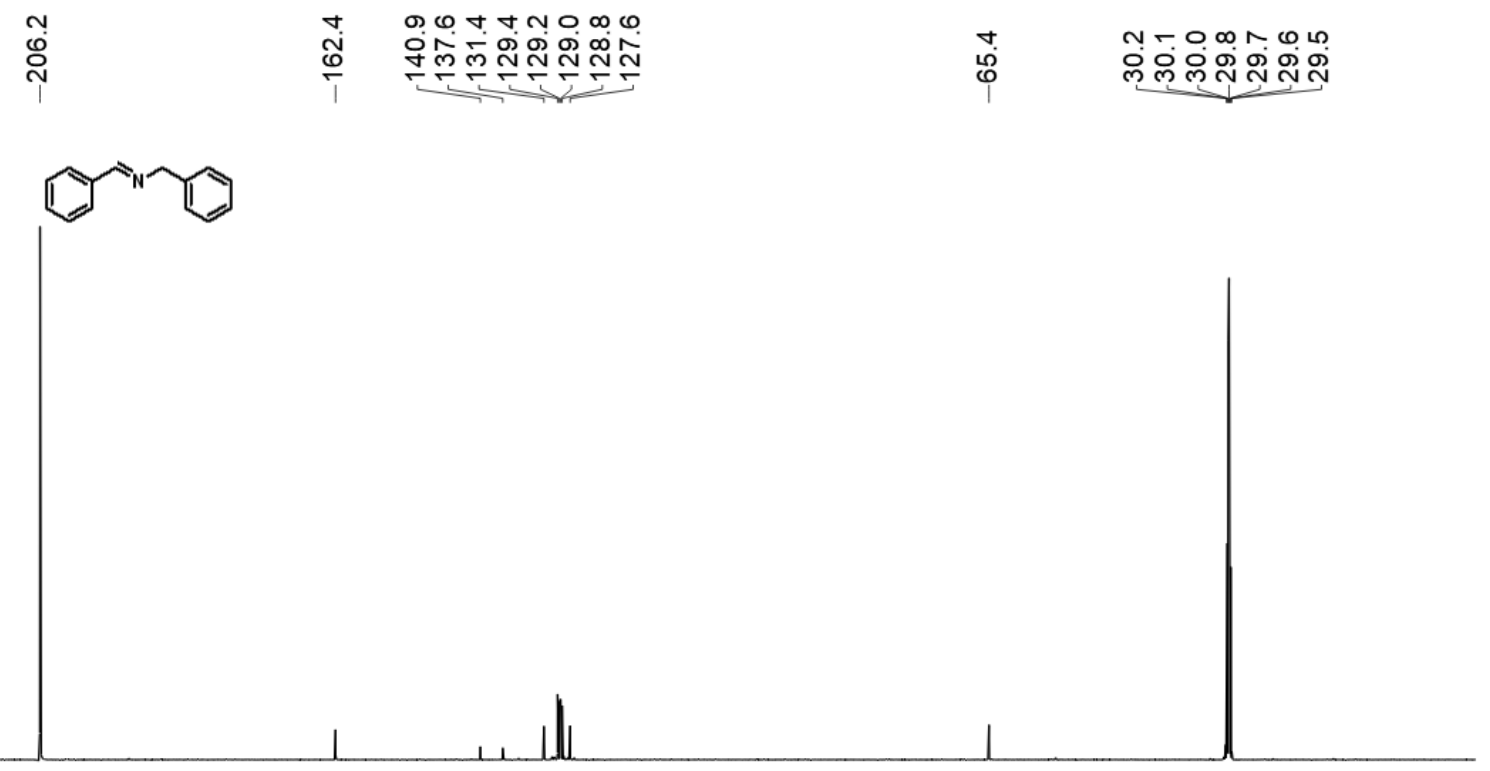

$\begin{array}{llllllllllllllllllllllll}210 & 200 & 190 & 180 & 170 & 160 & 150 & 140 & 130 & 120 & \begin{array}{c}110 \\ \mathrm{f} 1(\mathrm{ppm})\end{array} & 90 & 80 & 70 & 60 & 50 & 40 & 30 & 20 & 10 & 0\end{array}$

Figure S81. ${ }^{13} \mathrm{C}$ NMR spectrum of $\mathbf{5 a j}\left(\mathrm{CD}_{3} \mathrm{COCD}_{3}, 150 \mathrm{MHz}, 23{ }^{\circ} \mathrm{C}\right)$.

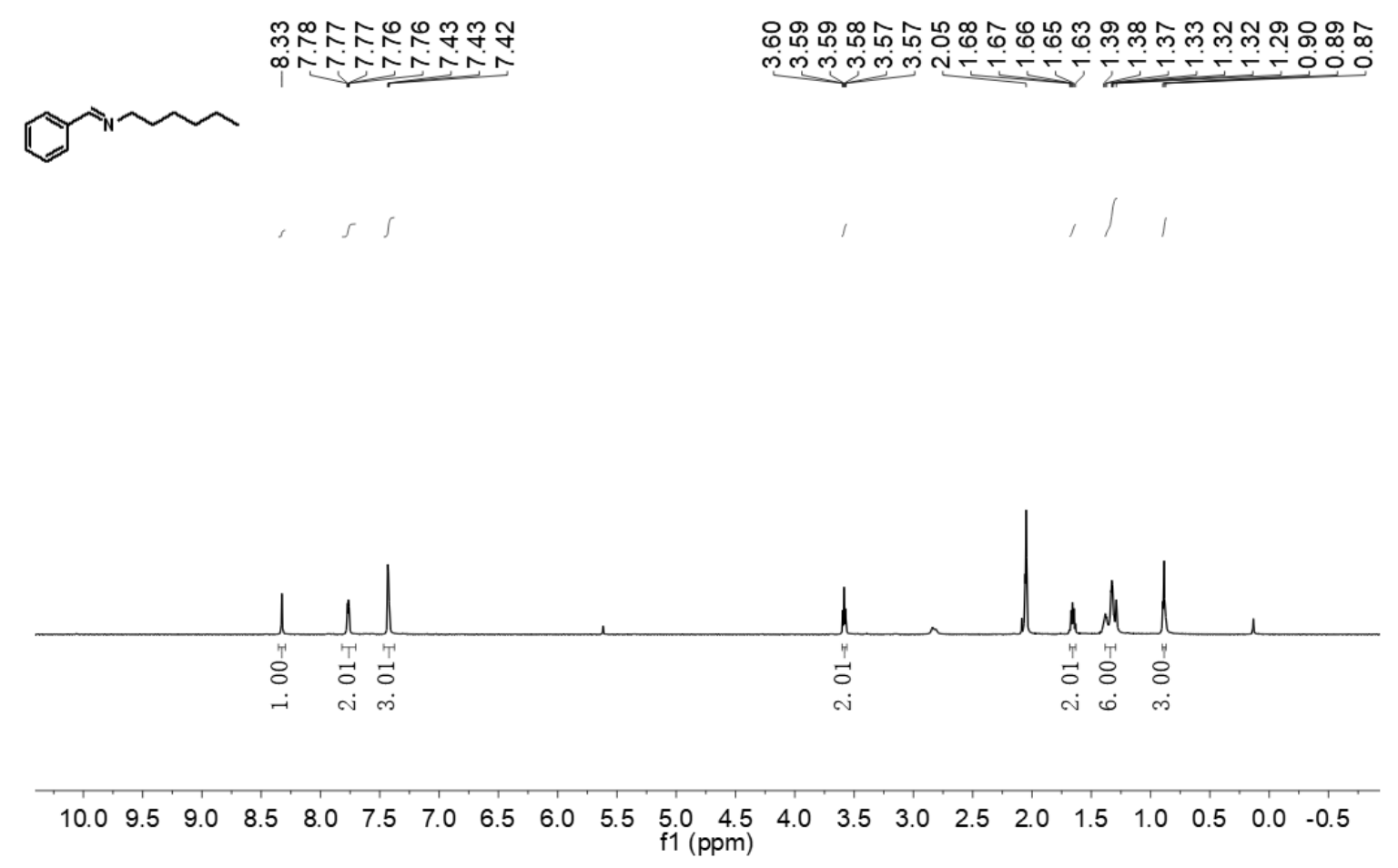

Figure S82. ${ }^{1} \mathrm{H}$ NMR spectrum of $5 \mathrm{ak}\left(\mathrm{CD}_{3} \mathrm{COCD}_{3}, 600 \mathrm{MHz}, 23{ }^{\circ} \mathrm{C}\right)$. 


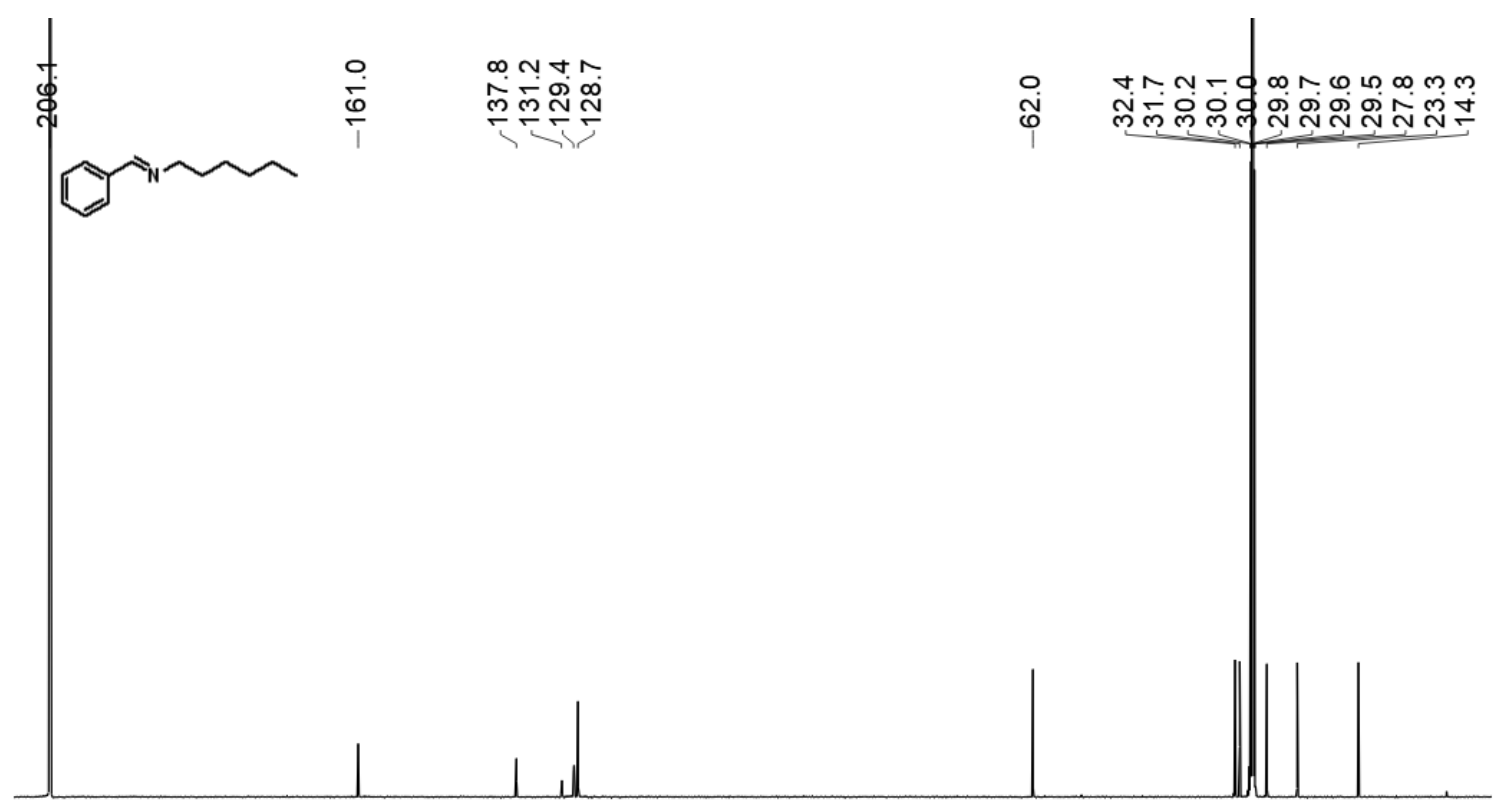

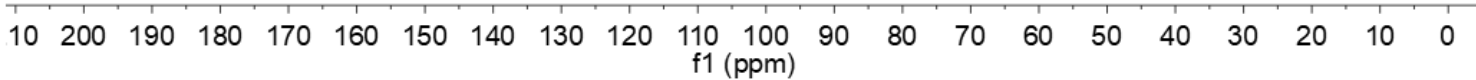

Figure S83. ${ }^{13} \mathrm{C}$ NMR spectrum of $5 \mathbf{a k}\left(\mathrm{CD}_{3} \mathrm{COCD}_{3}, 150 \mathrm{MHz}, 23{ }^{\circ} \mathrm{C}\right)$.

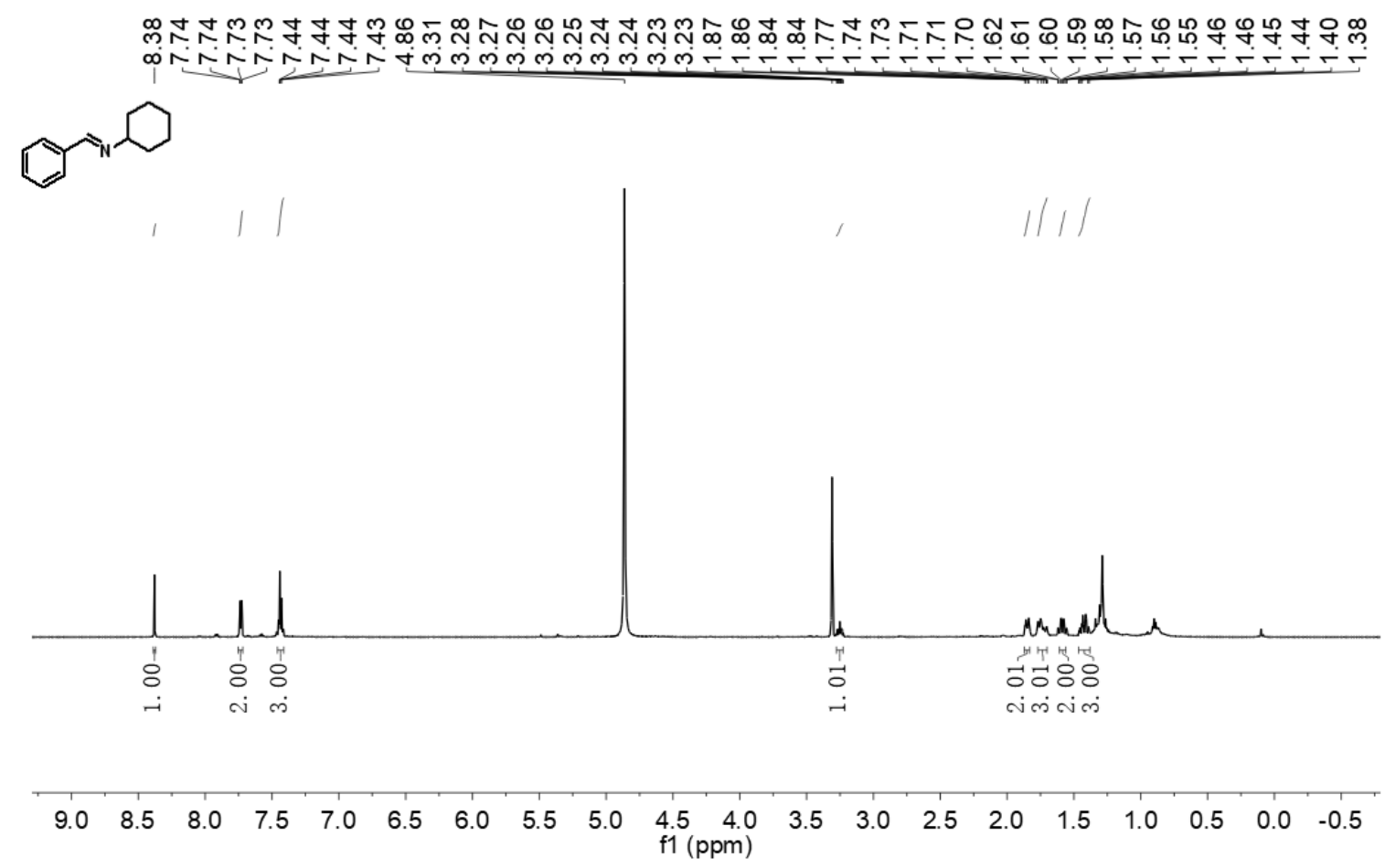

Figure S84. ${ }^{1} \mathrm{H}$ NMR spectrum of $\mathbf{5 a l}\left(\mathrm{CD}_{3} \mathrm{OD}, 600 \mathrm{MHz}, 23{ }^{\circ} \mathrm{C}\right)$. 


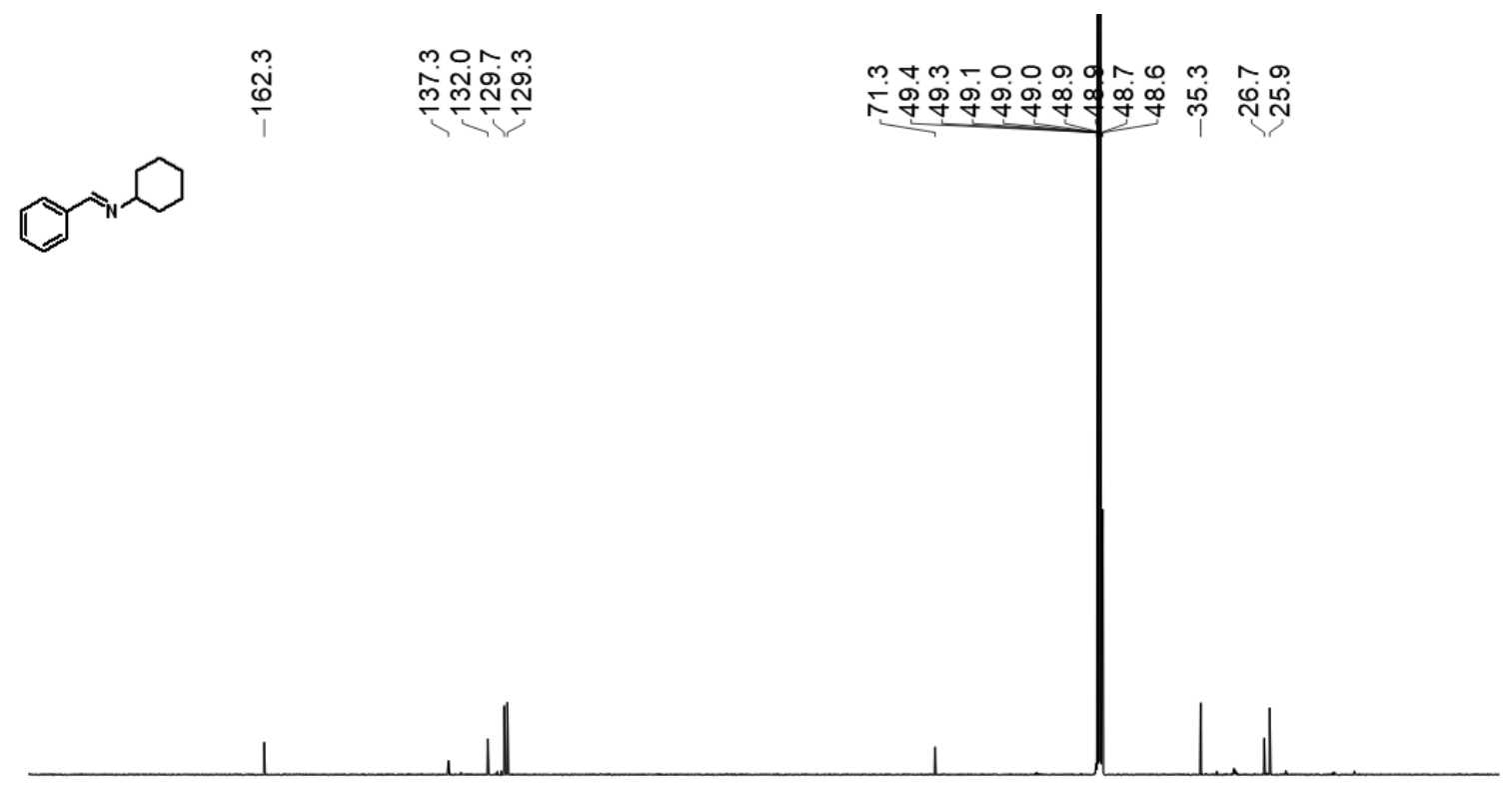

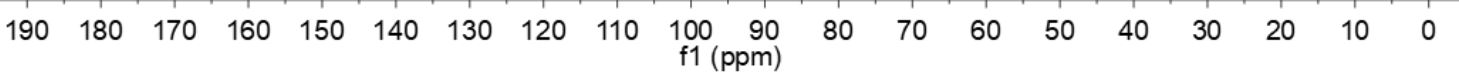

Figure S85. ${ }^{13} \mathrm{C}$ NMR spectrum of $\mathbf{5 a l}\left(\mathrm{CD}_{3} \mathrm{OD}, 150 \mathrm{MHz}, 23{ }^{\circ} \mathrm{C}\right)$.
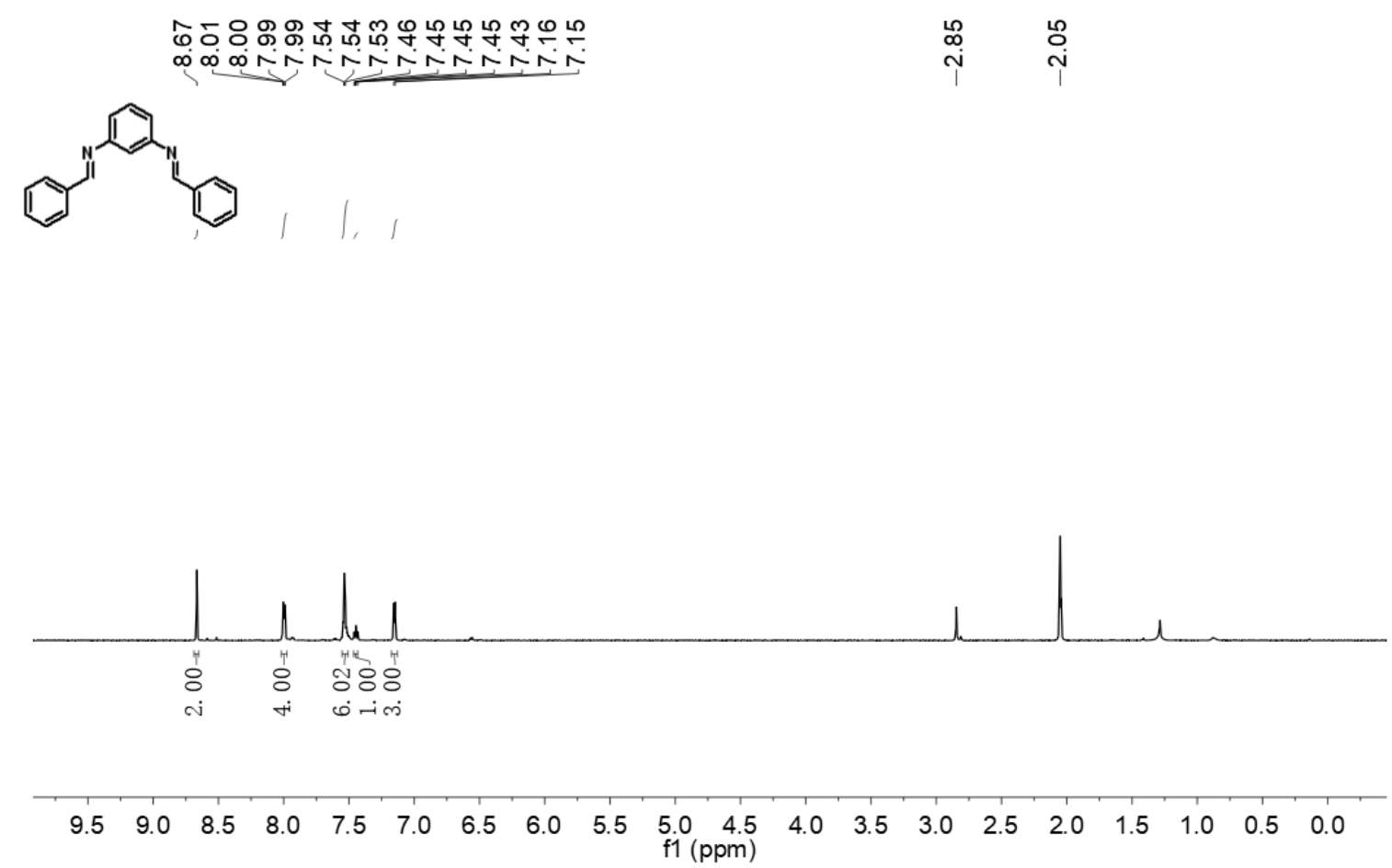

Figure S86. ${ }^{1} \mathrm{H}$ NMR spectrum of $5 \mathbf{a m}\left(\mathrm{CD}_{3} \mathrm{COCD}_{3}, 600 \mathrm{MHz}, 23{ }^{\circ} \mathrm{C}\right)$. 

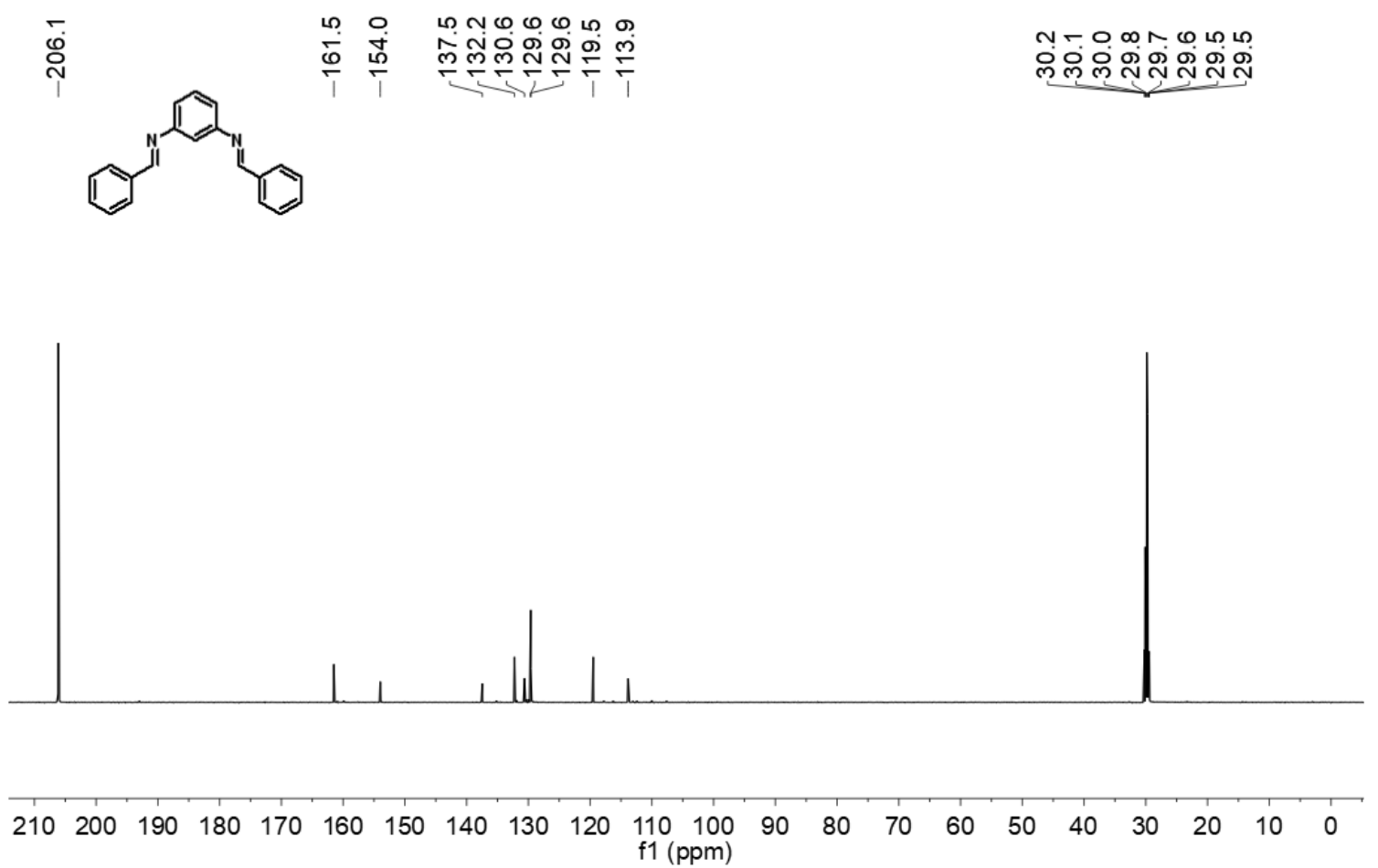

Figure S87. ${ }^{13} \mathrm{C}$ NMR spectrum of $5 \mathbf{a m}\left(\mathrm{CD}_{3} \mathrm{COCD}_{3}, 150 \mathrm{MHz}, 23{ }^{\circ} \mathrm{C}\right)$.

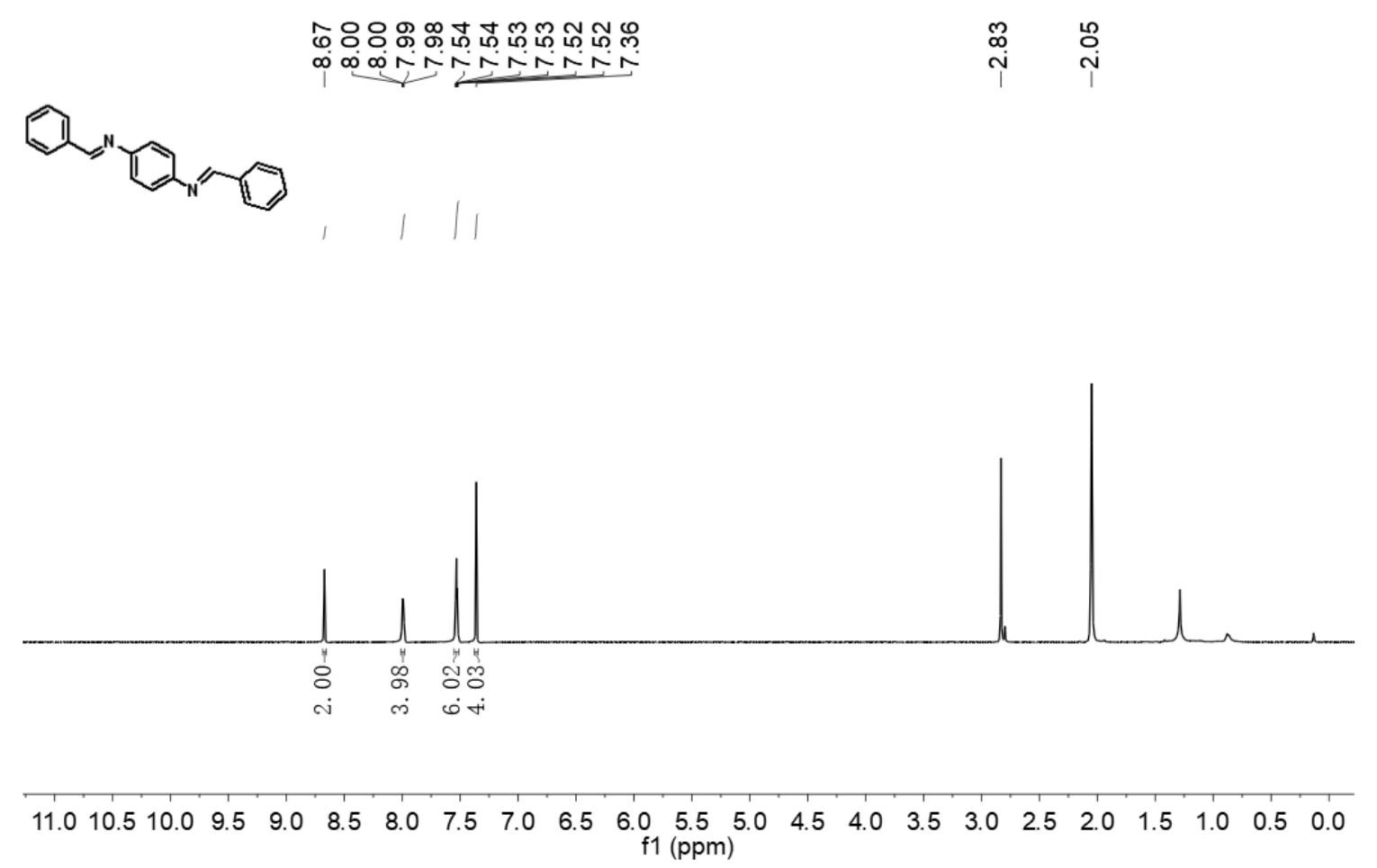

Figure S88. ${ }^{1} \mathrm{H}$ NMR spectrum of $\operatorname{5an}\left(\mathrm{CD}_{3} \mathrm{COCD}_{3}, 600 \mathrm{MHz}, 23{ }^{\circ} \mathrm{C}\right)$. 

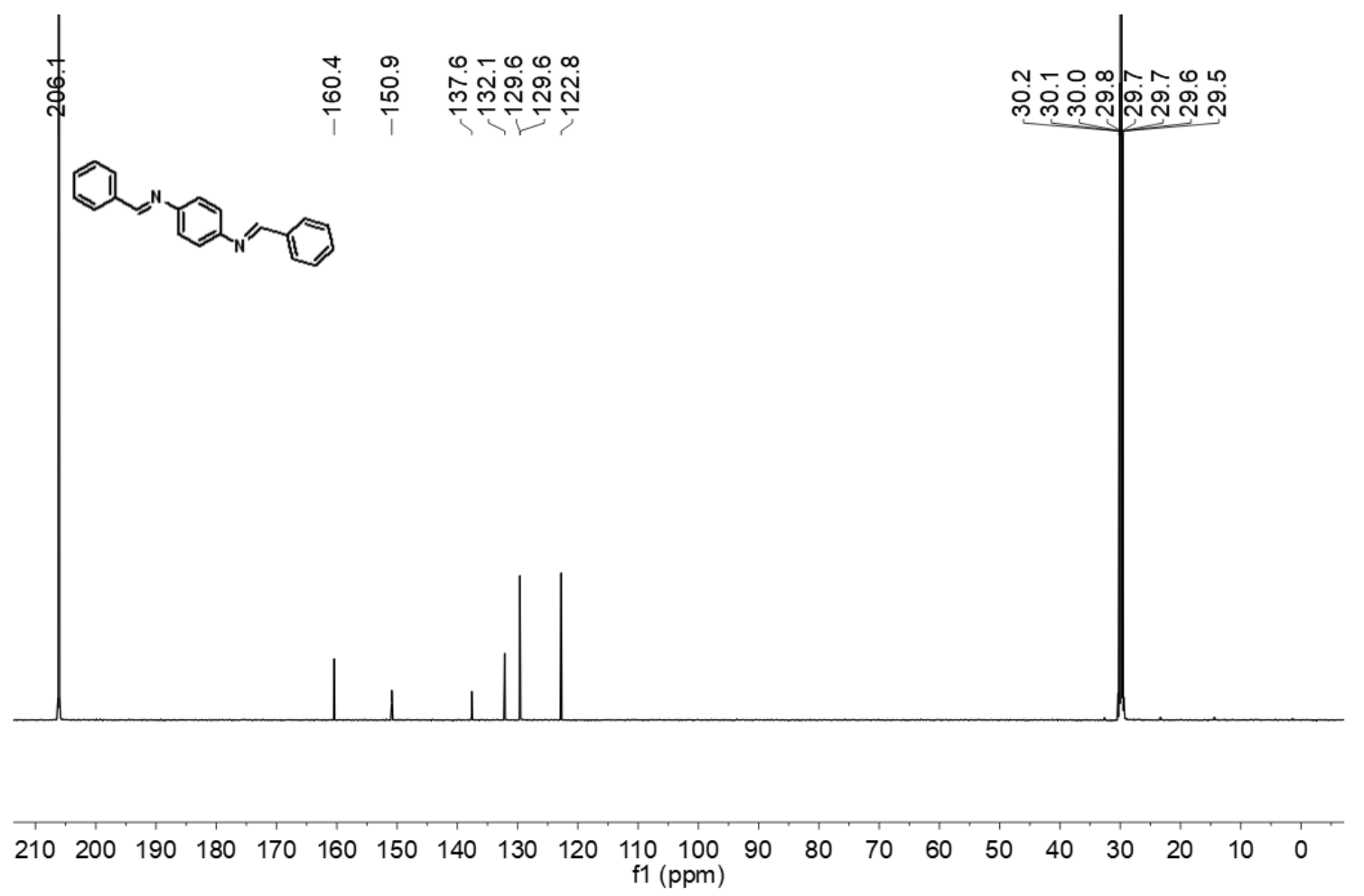

Figure S89. ${ }^{13} \mathrm{C}$ NMR spectrum of $\operatorname{5an}\left(\mathrm{CD}_{3} \mathrm{COCD}_{3}, 150 \mathrm{MHz}, 23{ }^{\circ} \mathrm{C}\right)$.

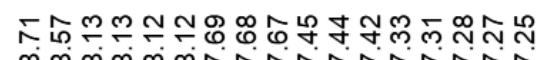

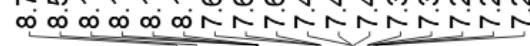

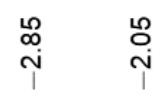<smiles>C(=N/c1ccccc1)\c1cccc(/C=N/c2ccccc2)c1</smiles>

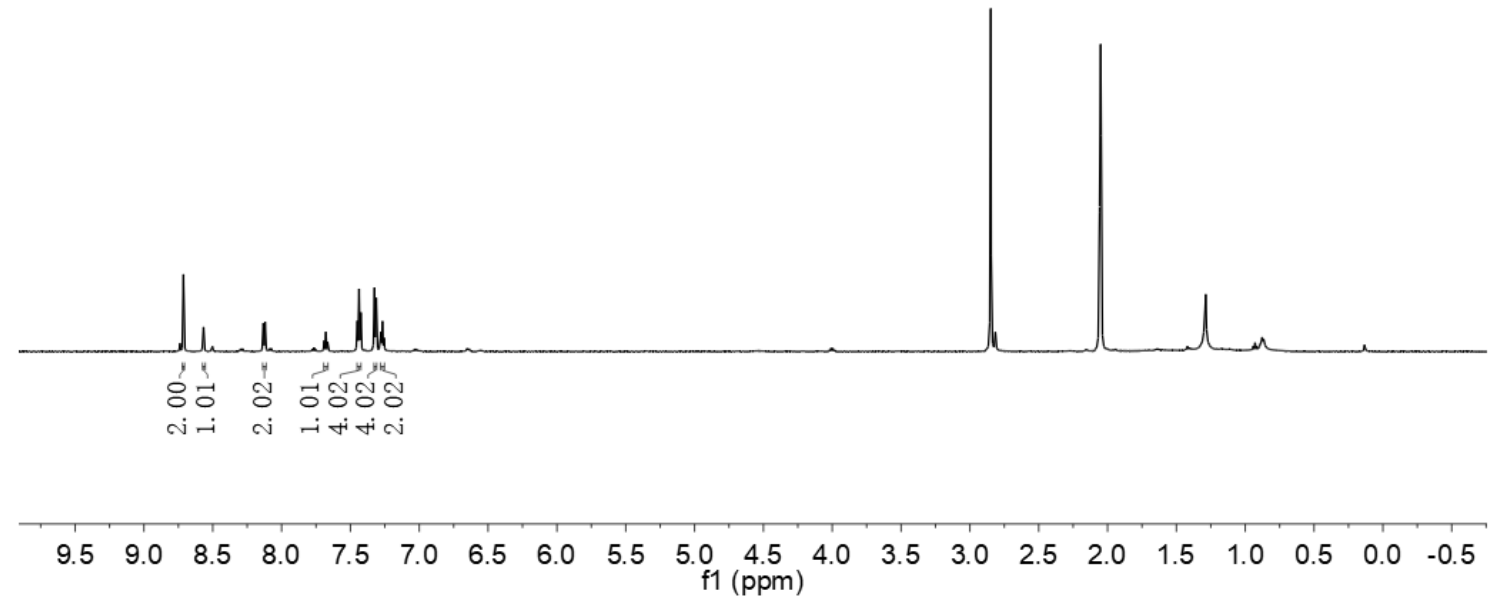

Figure S90. ${ }^{1} \mathrm{H}$ NMR spectrum of $\mathbf{5 a o}\left(\mathrm{CD}_{3} \mathrm{COCD}_{3}, 600 \mathrm{MHz}, 23{ }^{\circ} \mathrm{C}\right)$. 


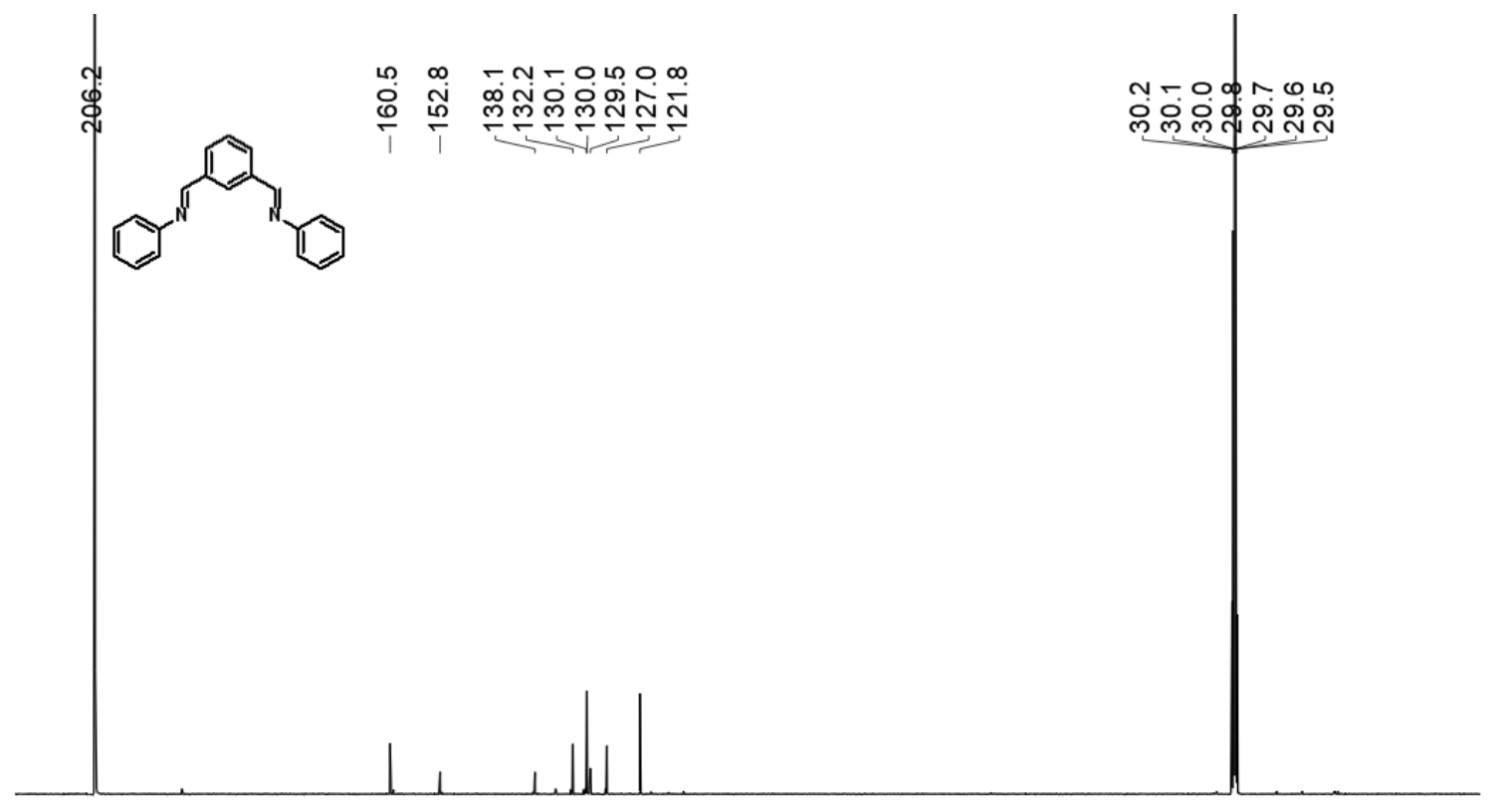

$\begin{array}{llllllllllllllllllllll}210 & 200 & 190 & 180 & 170 & 160 & 150 & 140 & 130 & 120 & \begin{array}{l}110 \\ \mathrm{f} 1(\mathrm{ppm})\end{array} & 90 & 80 & 70 & 60 & 50 & 40 & 30 & 20 & 10 & 0\end{array}$

Figure S91. ${ }^{13} \mathrm{C}$ NMR spectrum of $\mathbf{5 a o}\left(\mathrm{CD}_{3} \mathrm{COCD}_{3}, 150 \mathrm{MHz}, 23{ }^{\circ} \mathrm{C}\right)$.

\section{References}

(1) Yu, X.; Deng, L.; Zheng, B.; Zeng, B. R.; Yi, P.; Xu, X. A Spectroscopic Study on the Coordination and Solution Structures of the Interaction Systems Between Biperoxidovanadate Complexes and the Pyrazolylpyridine-like Ligands. Dalton. Trans. 2014, 43, 1524-1533.

(2) Robin, F.; Torsten, I.; Frederik, F.; Judith, Z.; Rhett, K. Manganese-Catalyzed and Base-Switchable Synthesis of Amines or Imines via Borrowing Hydrogen or Dehydrogenative Condensation. ACS Catal. 2018, 8, 8525-8630.

(3) Takahashi, Y.; Kondo, R.; Utsunomiya, M.; Suzuki, T.; Takeshita, H. T.; Obora, Y. Ti-Pd Alloys as Heterogeneous Catalysts for the Hydrogen Autotransfer Reaction and Catalytic Improvement by Hydrogenation Effects. ChemCatChem 2019, 11, 2432-2437.

(4) Saranya, S.; Ramesh, R.; Małecki, J. G. One-Pot Catalytic Approach for the Selective Aerobic Synthesis of Imines from Alcohols and Amines Using 
Efficient Arene Diruthenium(II) Catalysts under Mild Conditions. Eur. J. Org. Chem. 2017, 2017, 6726-6733.

(5) Rodríguez-Lugo, R. E.; Chacón-Terán, M. A.; De León, S.; Vogt, M.; Rosenthald, A. J.; Landaet, V. R. Synthesis, Characterization and $\mathrm{Pd}(\mathrm{II})$-Coordination Chemistry of the Ligand Tris(quinolin-8-yl)phosphite. Application in the Catalytic Aerobic Oxidation of Amines. Dalton Trans. 2018, 47, 2061-2072.

(6) Jin, Y.; Ke, Q.; Li, D.; Lei, Z.; Ling, Q.; Xu, J.; Cui, P. Nickel Doped Aluminophosphate-5 as an Efficient Heterogeneous Catalyst for Imine Synthesis by Direct Condensation of Alcohols and Amines at Room Temperature. ChemistrySelect 2018, 3, 2812-2818.

(7) Song, T.; Ren, P.; Duan, Y.; Wang, Z.; Chen, X.; Yang, Y. Cobalt Nanocomposites on N-doped Hierarchical Porous Carbon for Highly Selective Formation of Anilines and Imines from Nitroarenes. Green Chem. 2018, 20, $4629-4637$.

(8) Sliwa, M.; Mouton, N.; Ruckebusch, C.; Poisson, L.; Idrissi, A.; Aloïse, S.; Potier, L.; Dubois, J.; Poizat, O.; Buntinx, G. Investigation of Ultrafast Photoinduced Processes for Salicylidene Aniline in Solution and Gas Phase: Toward a General Photo-Dynamical Scheme. Photochem. Photobiol. Sci. 2010, 9, 661-669.

(9) Wang, K.; Gao, W.; Jiang, P.; Lan, K.; Yang, M.; Huang, X.; Ma, L.; Niu, F.; Li, R. Bi-functional Catalyst of Porous N-doped Carbon with Bimetallic $\mathrm{FeCu}$ for Solvent-Free Resultant Imines and Hydrogenation of Nitroarenes. Mol. Catal. 2019, 465, 43-53.

(10) Chen, B.; Shang, S.; Wang, L.; Zhang, Y.; Shuang, G. Mesoporous Carbon Derived from Vitamin $\mathrm{B}_{12}$ : a High-Performance Bifunctional Catalyst for Imine Formation. Chem. Commun. 2016, 52, 481-484.

(11) Zhang, M.; Wu, S.; Bian, L.; Cao, Q.; Fang, W. One-pot Synthesis of Pd-Promoted Ce-Ni Mixed Oxides as Efficient Catalysts for Imine Production from the Direct $N$-alkylation of Amine with Alcohol. Catal. Sci. Technol. 2019, 9, 286-301. 
(12) Cheng, S.; Ma, X.; Hu, Y.; Li, B. $\mathrm{MnO}_{2}$ /graphene Oxide: A Highly Efficient Catalyst for Imine Synthesis from Alcohols and Amines. Appl. Organomet. Chem. 2016, 31, e3659.

(13) Roth, H. G.; Romero, N. A.; Nicewicz, D. A. Experimental and Calculated Electrochemical Potentials of Common Organic Molecules for Applications to Single-Electron Redox Chemistry. Synlett 2016, 27, 714-723.

(14) Gopalaiah, K.; Saini, A. A Solvent-Free Process for Synthesis of Imines by Iron-Catalyzed Oxidative Self- or Cross-Condensation of Primary Amines Using Molecular Oxygen as Sole Oxidant. Catal. Lett. 2016, 146, 1648-1654.

(15) Sidır, Y.; Aslan, C.; Berber, H.; Sidır, İ. The Electronic Structure, Solvatochromism, and Electric Dipole Moments of New Schiff base Derivatives using Absorbance and Fluorescence Spectra. Struct. Chem. 2019, 30, 835-851.

(16) Geng, L.; Song, J.; Zhou, Y.; Xie, Y.; Huang, J.; Zhang, W.; Peng, L.; Liu, G. $\mathrm{CeO}_{2}$ Nanorods Anchored on Mesoporous Carbon as an Efficient Catalyst for Imine Synthesis. Chem. Commun. 2016, 52, 13495-13498.

(17) Dikusar, E.; Potkin, V.; Kozlov, N.; Yuvchenko, A. P. Synthesis of N,N'-bis-[3-alkoxy-4-(hydroxy, alkoxy, acyloxy)-phenylmethylene- and -Phenylmethyl]-1,3-phenylenediamines. Russ. J. Gen. Chem. 2009, 79, 258-263.

(18) Kang, Q.; Zhang, Y. Copper-catalyzed Highly Efficient Aerobic Oxidative Synthesis of Imines from Alcohols and Amines. Green Chem. 2012, 14, 1016-1019.

(19) Kaur, K. Hexafluoroacetone: An Appealing Key Player in Organic Chemistry. Synlett 2010, 15, 2363-2364.

(20) Manikandan, T. S.; Ramesh, R.; Shivalingegowda, N.; Krishnappagowda, L. N. Direct Aerobic Strategy for Synthesis of Imines via Alcohols and Amines Promoted by Ruthenium(II) ( $\eta^{6}$-p-cymene) Complexes. ChemistrySelect 2018, 3, 1561-1568.

(21) Han, L.; Xing, P.; Jiang, B. Selective Aerobic Oxidation of Alcohols to Aldehydes, Carboxylic Acids, and Imines Catalyzed by a Ag-NHC Complex. Org. Lett. 2014, 16, 3428-3431. 
(22) Eroglu, B.; Ozadali-Sari, K.; Unsal-Tan, O.; Dharmarajan, S.; Yogeeswari, P.; Balkan, A. Novel Thiazolidinone-Azole Hybrids: Design, Synthesis and Antimycobacterial Activity Studies. Iran. J. Pharm. Res. 2016, 5, 783-790. 